NBER WORKING PAPER SERIES

\title{
A SIMPLE NONPARAMETRIC ESTIMATOR FOR THE DISTRIBUTION OF RANDOM COEFFICIENTS
}

\author{
Patrick Bajari \\ Jeremy T. Fox \\ Kyoo il Kim \\ Stephen P. Ryan \\ Working Paper 15210 \\ http://www.nber.org/papers/w15210 \\ NATIONAL BUREAU OF ECONOMIC RESEARCH \\ 1050 Massachusetts Avenue \\ Cambridge, MA 02138 \\ August 2009
}

Bajari thanks the National Science Foundation, grant SES-0720463, for generous research support. Fox thanks the National Science Foundation, the Olin Foundation, and the Stigler Center for generous funding. Thanks to helpful comments from seminar participants at the AEA meetings, Chicago, Chicago GSB, UC Davis, European Commission antitrust, Far East Econometric Society meetings, LSE, Mannheim, MIT, Northwestern, Paris I, Quantitative Marketing and Economics, Queens, Rochester, Rutgers, Stanford, Stony Brook, Toronto, UCL, USC, Virginia, and Yale. Thanks to comments from Xiaohong Chen, Andrew Chesher, Philippe Fevrier, Amit Gandhi, Han Hong, David Margolis, Andrés Musalem, Peter Reiss, Jean-Marc Robin, Andrés Santos, Azeem Shaikh and Harald Uhlig. Thanks to research assistance from Chenchuan Li. The views expressed herein are those of the author(s) and do not necessarily reflect the views of the National Bureau of Economic Research.

NBER working papers are circulated for discussion and comment purposes. They have not been peerreviewed or been subject to the review by the NBER Board of Directors that accompanies official NBER publications.

(C) 2009 by Patrick Bajari, Jeremy T. Fox, Kyoo il Kim, and Stephen P. Ryan. All rights reserved. Short sections of text, not to exceed two paragraphs, may be quoted without explicit permission provided that full credit, including $\odot$ notice, is given to the source. 
A Simple Nonparametric Estimator for the Distribution of Random Coefficients

Patrick Bajari, Jeremy T. Fox, Kyoo il Kim, and Stephen P. Ryan

NBER Working Paper No. 15210

July 2009

JEL No. C01,C14,C25,C31,C35,I21,I28,L0,O1,O15

\begin{abstract}
$\underline{\text { ABSTRACT }}$
We propose a simple nonparametric mixtures estimator for recovering the joint distribution of parameter heterogeneity in economic models, such as the random coefficients logit. The estimator is based on linear regression subject to linear inequality constraints, and is robust, easy to program and computationally attractive compared to alternative estimators for random coefficient models. We prove consistency and provide the rate of convergence under deterministic and stochastic choices for the sieve approximating space. We present a Monte Carlo study and an empirical application to dynamic programming discrete choice with a serially-correlated unobserved state variable.
\end{abstract}

Patrick Bajari

Professor of Economics

University of Minnesota

4-101 Hanson Hall

1925 4th Street South

Minneapolis, MN 55455

and NBER

bajari@econ.umn.edu

Jeremy T. Fox

Department of Economics

University of Chicago

1126 East 59th Street

Chicago, IL 60637

and NBER

fox@uchicago.edu

\author{
Kyoo il Kim \\ Department of Economics \\ University of Minnesota \\ 4-129 Hanson Halll \\ 1925 4th Street South \\ Minneapolis, MN 55455 \\ kyookim@umn.edu \\ Stephen P. Ryan \\ MIT Department of Economics \\ E52-262C \\ 50 Memorial Drive \\ Cambridge, MA 02142 \\ and NBER \\ sryan@mit.edu
}




\section{Introduction}

In economics, it is common to observe that otherwise identical agents behave differently when faced with identical choice environments, due to such factors as heterogeneity in preferences. A growing econometric literature has addressed this problem by providing estimators that allow the coefficients of the economic model to vary across agents (for recent work in demand estimation, see Berry, Levinsohn, and Pakes (1995), Nevo (2001), Petrin (2002), and Rossi, Allenby, and McCulloch (2005)). In this paper, we describe a general method for estimating such random coefficient models that is both nonparametric with respect to the underlying distribution of heterogeneity and easy to compute. Our estimator exploits a reparameterization of the underlying model so that the parameters enter linearly. This is in contrast to previous approaches in the literature, such as the EM algorithm, Markov Chain Monte Carlo (MCMC), simulated maximum likelihood and simulated method of moments, which are highly nonlinear. Linearity simplifies the computation of the parameters.

To motivate our approach, consider the following simple example. Suppose that the econometrician is interested in estimating a binary logit model with a scalar random coefficient. Let $y=1$ if the first option is chosen and let $y=0$ otherwise. Suppose that this model has a single independent variable, $x$. Furthermore, suppose that the random coefficient is known to have support on the $[0,1]$ interval. Fix a large but finite grid of $R$ equally spaced points. Suppose

that the grid points take on the values $\frac{1}{R}, \frac{2}{R}, \ldots, \frac{R-1}{R}, 1$. The parameters of our model are $\theta^{r}$, the frequencies of each random coefficient $\frac{r}{R}$. It then follows that the empirical probability that the dependent variable $y$ is 1 conditional on $x$ can be approximated by the linear combination

$$
\operatorname{Pr}(y=1 \mid x) \approx \sum_{r=1}^{R} \theta^{r} \frac{\exp \left(\frac{r}{R} \cdot x\right)}{1+\exp \left(\frac{r}{R} \cdot x\right)}
$$

The key insight of our approach is that the dependent variable in our model, $\operatorname{Pr}(y=1 \mid x)$, is linearly related to the model parameters $\theta^{r}$, irrespective of the nonlinear model used to compute the probability under a given type $r$. Instead of optimizing over that nonlinear model, we compute the probability under each type as if it were the true parameter, and then find the proper mixture of those models that best approximates the actual data. In the paper, we demonstrate that the $\theta^{r}$ parameters can be consistently estimated using inequality constrained least squares. This estimator has a single, global optimum and widely-available specialized minimization approaches are guaranteed to converge to that point. This contrasts with alternative approaches 
to estimating random coefficient models, where the objective functions can have multiple local optima and the econometrician is not guaranteed to find the global solution.

We also note that our approach does not require a parametric specification for the distribution of the random coefficients. We can closely approximate any well behaved distribution on $[0,1]$ by making $R$ large and by choosing $\theta^{r}$ appropriately. Intuitively, our framework allows us to manipulate the cell size in a histogram. Many alternative estimators require computationally expensive nonlinear optimization, and as a result researchers frequently use tightly specified parametric models in applied work because of computational constraints. For example, applied researchers frequently assume that the random coefficients are mutually independent and normal. Our approach allows us to estimate the joint distribution of random coefficients without having to impose restrictions on the family of distributions.

We show how to extend this simple intuition to a more general framework. First, modeling the random coefficients using equally spaced grid points does not lead to a smooth estimated density. We suggest alternative methods for discretizing the model that give smooth densities, while still maintaining the linear relationship between the dependent variable and the model parameters. Second, we discuss how our approach can be extended to more complex economic choice models. As an example, we show how to include a nonparametric distribution of random coefficients in a dynamic programming discrete choice model such as Rust (1987). In our empirical application, we demonstrate that our approach can dramatically reduce the computational burden of these models. Third, we extend our approach to accommodate the case where the support of the basis functions is not known, and the econometrician must search over the parameter space.

Our approach can include random coefficients on multiple independent variables. Because of the curse of dimensionality in estimating infinite dimensional objects, the rate of convergence of the estimator of the distribution (with a deterministic choice of grid points) is inversely related to the number of random coefficients. With a stochastic choice of grid points, the rate of convergence is invariant to the number of random coefficients.

We do not claim that our estimator dominates all existing methods in all potential demandestimation applications. In the paper, we discuss some strengths and weaknesses of our approach. First, if the researcher has aggregate data on market shares with price endogeneity, the approach of Berry, Levinsohn and Pakes (1995) is likely to be preferable. Our approach can accommodate aggregate data, even if market shares are measured with error. However, we need to specify a reduced-form pricing function as in Petrin and Train (2009) in order to account for price endogeneity. While this approach has worked well in some examples, it is not possible to prove the existence of this pricing function in the general case. Second, in small samples, Bayesian 
methods that use prior information will likely have better finite-sample performance (Rossi, Allenby and McCulloch 2005; Burda, Harding and Hausman 2008). Our methods are intended for applications where the researcher has access to large sample sizes.

Our approach is a general, nonparametric mixtures estimator. The most common frequentist, nonparametric estimator is nonparametric maximum likelihood or NPMLE (Laird 1978, Böhning 1982, Lindsay 1983, Heckman and Singer 1984). Often the EM algorithm is used for computation (Dempster, Laird and Rubin 1977), but this approach is not guaranteed to find the global maximum. The literature worries about the strong dependence of the output of the EM algorithm on initial starting values and well as the difficulty in diagnosing convergence (Seidel, Mosler and Alker 2000, Verbeek, Vlassis and Kröse 2002, Biernacki, Celeux and Govaert 2003, Karlis and Xekalaki 2003). ${ }^{1}$ Further, the EM algorithm has a slow rate of convergence even when it does converge to a global solution (Pilla and Lindsay 2001). Li and Barron (2000) introduce another alternative, but again our approach is computationally simpler. ${ }^{2}$ The discrete-grid idea (called the "histogram" approach) is found outside of economics in Kamakura (1991), who uses a discrete grid to estimate an ideal-point model. He does not discuss identification, the nonparametric statistical properties of his approach, or any of our extensions. Of course, mixtures themselves have a long history in economics (Quandt and Ramsey 1978).

We prove the consistency of our estimator for the distribution of random parameters, in function space and under a potential ill-posed inverse problem. We note that many if not most of the alternative estimators discussed above do not have general, nonparametric consistency theorems for the estimator for the distribution of random parameters. Our consistency theorem is not specific to the economic model being estimated.

The outline of our paper is as follows. In section 2, we start by precisely describing our model and estimator. We focus on the motivating example of discrete choice with individual data. However, we provide other examples such as discrete choice with aggregate data, dynamic

\footnotetext{
${ }^{1}$ Another drawback of NPMLE that is specific to mixtures of normal distributions, a common approximating choice, is that the likelihood is unbounded and hence maximizing the likelihood does not produce a consistent estimator. There is a consistent root but it is not the global maximum of the likelihood function (McLachlan and Peel 2000).

${ }^{2}$ There is a literature on customized estimators for particular economic models as opposed to general mixtures estimators. For discrete choice models, Ichimura and Thompson (1998) and Gautier and Kitamura (2008) provide estimators for models with two choices and where covariates enter linearly. Lewbel (2000) considers the identification and estimation of mean preferences using a special regressor and a mean independence assumption. Manski's (1975) maximum score estimator is consistent for mean preferences in the presence of random coefficients for the case of two choices only. Briesch, Chintagunta and Matzkin (2007) allow utility to be a nonparametric function of $x$ and a scalar unobservable. Hoderlein, Klemelä and Mammen (2008) estimate the distribution of heterogeneity in a linear regression model.
} 
discrete choice, mixed continuous and discrete choice (selection), and discrete choice models with endogenous regressors. Following Chen and Pouzo (2009a, 2009b), we view our estimator as a sieve estimation problem. In section 3 , we prove consistency in a function space if the true distribution is any arbitrary distribution function. In section 4, under additional assumptions we derive a pointwise rate of convergence for our distribution estimator. In section 5 , we discuss how to conduct inference under the assumption that the set of types used in estimation is the true set of types. In section 6, we conduct a Monte Carlo experiment to investigate the finite sample performance of our estimator. In section 7 , we apply our estimator to a dynamic programming, discrete choice empirical problem studied in Duflo, Hanna and Ryan (2008). The dynamic programming problem has a serially correlated, unobserved state variable. We allow for a nonparametric distribution of random parameters. Code that implements our estimator is available on the internet. ${ }^{3}$

\section{The Estimator}

\subsection{General Notation}

We first introduce general notation. The econometrician observes a real valued vector of covariates $x$. The dependent variable in our model is denoted $y$. In our examples, we will focus primarily on the case where the range of $y$ is a finite number of integer values as is customary in discrete choice models. However, much of our analysis extends to the case where $y$ is real valued.

Let $A$ denote a (measurable) set in the range of $y, \mathcal{Y}$. We let $P_{A}(x)$ denote the probability that $y \in A$ when the decision problem has characteristics $x$. Let $\beta$ denote a random coefficient. In our framework, this is a finite-dimensional, real-valued vector. We let $g_{A}(x, \beta)$ be the probability of $A$ conditional on the random coefficients $\beta$ and characteristics $x$. The density and CDF of the random coefficients are denoted as $f(\beta)$ and $F(\beta)$ respectively. The function $g_{A}$ is specified as a modeling primitive. In our simple example in the introduction, $g_{A}$ corresponds to the logit model. Given these definitions it follows that

$$
P_{A}(x)=\int g_{A}(x, \beta) d F(\beta) .
$$

On the right hand side of the above equation, $g_{A}(x, \beta)$ gives the probability of $A$ conditional on $x$ and $\beta$. We average over the distribution of $\beta$ using the $\operatorname{CDF} F(\beta)$ to arrive at $P_{A}(x)$, the

\footnotetext{
${ }^{3}$ The code can be accessed at http://home.uchicago.edu/ fox .
} 
population probability of the event $A$ conditional on $x$.

In our framework, the object the econometrician wishes to estimate is $F(\beta)$, the distribution of random coefficients. Identification means that a unique $F(\beta)$ solves (1) for all $x$ and all $A .^{4}$

More technical readers may prefer functional operator notation. Let $L$ be an operator that takes the distribution function $F$ as an argument and produces the probability function $\mathcal{P}=$ $\left\{P_{A}(\cdot) \forall A\right.$ \}, where $\mathcal{P}$ is $P_{A}(x)$ for all valid $A$ and $x$. With a limiting dataset, the econometrician can identify $\mathcal{P}$ using observations on pairs $(x, y)$. Let (1), for all valid $x$ and $A$, be written as $\mathcal{P}=L(F)$. Identification of the distribution means $L^{-1}$ is a function. A restatement of the definition of identification is that $L$ is one-to-one in $F$ : each valid $F$ produces a different $\mathcal{P}$.

This paper focuses on estimating $F$ using a finite dataset $\left(x_{i}, y_{i}\right)$ for $i=1, \ldots, N$ and not identification. The proof of consistency of our estimator will address the potential ill-posedness of $L$ : the inverse of $L, L^{-1}$, may not be continuous in $x$, when $A$ is fixed.

\subsection{The Logit Model}

As we discussed in the introduction, the motivating example for our paper is the logit with random coefficients. We shall begin by discussing this example in detail. Afterwards, we will show how our approach extends to other random coefficient models including dynamic discrete choice and demand models where the dependent variable is represented by a vector of discrete and continuous variables. The estimation method that we propose, however, can in principal be applied to any model that can be written in the form (1).

In the logit model, agents $i=1, \ldots, N$ can choose between $j=1, \ldots, J$ mutually exclusive alternatives. The exogenous variables for choice $j$ are in the $K \times 1$ vector $x_{i, j}$. In the example of demand estimation, $x_{i, j}$ might include the non-price product characteristics, the price of good $j$ and the demographics of agent $i$. We shall let $x_{i}=\left(x_{i, 1}^{\prime}, \ldots, x_{i, J}^{\prime}\right)$ denote the stacked vector of the $J x_{i, j}$ 's, the observable characteristics.

In the model, there are $r=1, \ldots, R$ types of agents. The unobservable preference parameters of type $r$ are equal to the $K \times 1$ vector $\beta^{r}$. We shall discuss how to choose the types $\beta^{r}$ below. For the moment, assume that the $\beta^{r}$ are fixed and exogenously specified. As in our example in the introduction, it might be helpful to think of the $\beta^{r}$ being defined using a fixed grid on a compact set. Later we will view the $R$ types of consumers as giving a nonparametric, sieve approximation to an arbitrary distribution for $\beta, F(\beta)$. The random variable $\beta$ is distributed

\footnotetext{
${ }^{4}$ This is the definition used in certain relevant papers on identification in the statistics literature, see Teicher (1963).
} 
independently of $x$. The probability of type $r$ in the population is $\theta^{r}$. Let $\theta=\left(\theta^{1}, \ldots, \theta^{R}\right)^{\prime}$ denote the corresponding vector. This will be a parameter in our model. The $\theta$ must lie on the simplex, or

$$
\begin{aligned}
& \sum_{r=1}^{R} \theta^{r}=1 \\
& \theta^{r} \geq 0 .
\end{aligned}
$$

If agent $i$ is of type $r$, her utility for choosing good $j$ is equal to

$$
u_{i, j}=x_{i, j}^{\prime} \beta^{r}+\epsilon_{i, j}
$$

There is an outside good with utility $u_{i, 0}=\varepsilon_{i, 0}$. Assume that $\epsilon_{i, j}$ is distributed as Type I extreme value and is independent of $x_{i}$ and $\beta^{r}$. Agents in the model are assumed to be utility maximizers. The observable dependent variable $y_{i, j}$ is generated as

$$
y_{i, j}= \begin{cases}1 & \text { if } u_{i, j}>u_{i, j^{\prime}} \text { for all } j^{\prime} \neq j \\ 0 & \text { otherwise }\end{cases}
$$

Let $y_{i}$ be the vector $\left(y_{i, 1}, \ldots, y_{i, J}\right)$. The probability of type $r$ picking choice $j$ at $x_{i}$ is

$$
g_{j}\left(x_{i}, \beta^{r}\right)=\frac{\exp \left(x_{i, j}^{\prime} \beta^{r}\right)}{1+\sum_{j^{\prime}=1}^{J} \exp \left(x_{i, j^{\prime}}^{\prime} \beta^{r}\right)} .
$$

Because the error term is extreme value, it follows that

$$
\operatorname{Pr}\left(y_{i, j}=1 \mid x_{i}\right)=\sum_{r=1}^{R} \theta^{r} g_{j}\left(x_{i}, \beta^{r}\right)=\sum_{r=1}^{R} \theta^{r} \frac{\exp \left(x_{i, j}^{\prime} \beta^{r}\right)}{1+\sum_{j^{\prime}=1}^{J} \exp \left(x_{i, j^{\prime}}^{\prime} \beta^{r}\right)} .
$$

\subsection{Linear Regression}

We study the estimation problem: the researcher has $i=1, \ldots, N$ observations on $\left(x_{i}, y_{i}\right)$. A first method for estimating the parameters $\theta$ is by ordinary least squares. To construct the estimator, begin by adding $y_{i, j}$ to both sides of (4) and moving $\operatorname{Pr}\left(y_{i, j}=1 \mid x_{i}\right)$ to the right side 
of this equation, which gives

$$
y_{i, j}=\left(\sum_{r=1}^{R} \theta^{r} g_{j}\left(x_{i}, \beta^{r}\right)\right)+\left(y_{i, j}-\operatorname{Pr}\left(y_{i, j}=1 \mid x_{i}\right)\right) .
$$

Define the $R \times 1$ vector $z_{i, j}=\left(z_{i, j, 1}, \ldots, z_{i, j, R}\right)^{\prime}$ with individual elements $z_{i, j, r}=g_{j}\left(x_{i}, \beta^{r}\right)$ and let $z_{i}=\left(z_{i, 1}, \ldots, z_{i, J}\right)$ be the stacked vector of $z_{i, j}$ 's. Recall that $\beta^{r}$ is fixed and is not a parameter to be estimated. As a result, given $x_{i}$, the term $z_{i, j, r}$ is a known constant. Next, by the definition of a choice probability,

$$
E\left[y_{i, j}-\operatorname{Pr}\left(y_{i, j}=1 \mid x_{i}\right) \mid z_{i}\right]=0 .
$$

This implies that the following ordinary least squares problem is a consistent estimator of the $\theta^{r}$

$$
\widehat{\theta}=\arg \min _{\theta} \frac{1}{N J} \sum_{i=1}^{N} \sum_{j=1}^{J}\left(y_{i, j}-z_{i, j}^{\prime} \theta\right)^{2} .
$$

Let $Y$ denote the $N J \times 1$ vector formed by stacking the $y_{i, j}$ and let $Z$ be the $N J \times R$ matrix formed by stacking the $z_{i, j}$. Then our estimator is $\widehat{\theta}=\left(Z^{\prime} Z\right)^{-1} Z^{\prime} Y{ }^{5}$

Equation (5) implies that the standard exogeneity condition is satisfied for least squares. The "error term" in our least squares regression is $y_{i, j}-\operatorname{Pr}\left(y_{i, j}=1 \mid x_{i}\right)$. This is the difference between the $y_{i, j}$ and the probability that $y_{i, j}=1$. This prediction error only depends on the realization of the $\varepsilon_{i, j}$ 's, which are i.i.d. and as a consequence independent of $z_{i}$. The least squares estimator will have a unique solution so long as $Z$ has rank $R$.

\subsection{Inequality Constrained Linear Least Squares}

A limitation of the ordinary least squares estimator is that $\widehat{\theta}$ need not satisfy (2) and (3). In practice, one might wish to constrain $\widehat{\theta}$ to be a well-defined probability measure. This would be useful in making sure that our model predicts probabilities that always lie between zero and one. Also, this may be important if the economist wishes to interpret the distribution of $\beta$ as a

\footnotetext{
${ }^{5}$ We focus on the least squares criterion, rather than a likelihood or pseudo-likelihood, for computational simplicity. However, it is not necessarily the case that the least squares criterion will result in a less efficient estimator. The least squares objective function enforces all of the parametric assumptions inherent in the logit model, which arises in the definition of $z_{i, j, r}$. There is no sense in which the likelihood alternative uses more structure. Second, in a nonparametric world, the true distribution lies in an infinite-dimensional space, and the limiting distribution of any estimator for the infinite-dimensional object is not known. Therefore, we avoid discussing efficiency, which relies on the estimator having an asymptotically normal distribution.
} 
structural parameter. We suggest estimating $\theta$ using inequality constrained least squares,

$$
\widehat{\theta}=\arg \min _{\theta} \frac{1}{N J} \sum_{i=1}^{N} \sum_{j=1}^{J}\left(y_{i, j}-z_{i, j}^{\prime} \theta\right)^{2}
$$

subject to $(2)$ and (3).

This minimization problem is a quadratic programming problem subject to linear inequality constraints. ${ }^{6}$ The minimization problem is convex and routines like MATLAB's lsqlin guarantee finding a global optimum. One can construct the estimated cumulative distribution function for the random coefficients as

$$
\hat{F}(\beta)=\sum_{r=1}^{R} \hat{\theta}^{r} 1\left[\beta^{r} \leq \beta\right],
$$

where $1\left[\beta^{r} \leq \beta\right]$ is equal to 1 when $\beta^{r} \leq \beta$. Thus, we have a structural estimator for a distribution of random parameters in addition to a flexible method for approximating choice probabilities.

\subsection{Smooth Basis Densities}

A limitation of the method above is that the CDF of the random parameters will be a step function. In applied work, it is often attractive to have a smooth distribution of random parameters. In this subsection, we describe one approach to estimating a density instead of a CDF. Our approach is easily extended to this case: instead of modeling the distribution of the random parameters as a mixture of point masses, we instead model the density as a mixture of normal densities.

Let a basis $r$ be a normal distribution with mean the $K \times 1$ vector $\mu^{r}$ and standard deviation the $K \times 1$ vector $\sigma^{r}$. Let $N\left(\beta_{k} \mid \mu_{k}^{r}, \sigma_{k}^{r}\right)$ denote the normal density of the $k$ th random parameter. Under normal basis functions, the joint density for a given $r$ is just the product of the marginals, or

$$
N\left(\beta \mid \mu^{r}, \sigma^{r}\right)=\prod_{k} N\left(\beta_{k} \mid \mu_{k}^{r}, \sigma_{k}^{r}\right)
$$

Let $\theta^{r}$ denote the probability weight given to the $r$ th basis, $N\left(\beta \mid \mu^{r}, \sigma^{r}\right)$. As in the previous

\footnotetext{
${ }^{6}$ Lindsay (1983) discusses how if there are at most $M$ distinct values of the $N$ observations $\left(x_{i}, y_{i}\right)$, then the nonparametric maximum likelihood estimator can maximize the likelihood using at most $M$ points of support for the beta space, $\beta$. Adding more points of support would not increase the statistical fit given the finite sample. Here, we fix the points of support and estimate only the weights, so we do not appeal to Lindsay's results. In our simulations and empirical work, we often find that only a few of the many points of support have nonzero weights in the final estimates.
} 
subsection, it is desirable to constrain $\theta$ to lie in the simplex, that is, (2) and (3).

For a given $r$, make $S$ simulation draws from $N\left(\beta \mid \mu^{r}, \sigma^{r}\right)$. Let a particular draw $s$ be denoted as $\beta^{r, s}$. We can then simulate $\operatorname{Pr}\left(y_{i, j}=1 \mid x_{i}\right)$ as:

$$
\operatorname{Pr}\left(y_{i, j}=1 \mid x_{i}\right) \approx \sum_{r=1}^{R} \theta^{r}\left(\frac{1}{S} \sum_{s=1}^{S} g_{j}\left(x_{i}, \beta^{r, s}\right)\right)=\sum_{r=1}^{R} \theta^{r}\left(\frac{1}{S} \sum_{s=1}^{S} \frac{\exp \left(x_{i, j}^{\prime} \beta^{r, s}\right)}{1+\sum_{j^{\prime}=1}^{J} \exp \left(x_{i, j^{\prime}}^{\prime} \beta^{r, s}\right)}\right) .
$$

We use the $\approx$ to emphasize from now on that we work with sieve approximations in finite samples. We can then estimate $\theta$ using the inequality constrained least squares problem

$$
\widehat{\theta}=\arg \min _{\theta} \frac{1}{N J} \sum_{i=1}^{N} \sum_{j=1}^{J}\left(y_{i, j}-\sum_{r=1}^{R} \theta^{r}\left(\frac{1}{S} \sum_{s=1}^{S} g_{j}\left(x_{i}, \beta^{r, s}\right)\right)\right)^{2}
$$

subject to $(2)$ and $(3)$.

This is once again inequality constrained, linear least squares, a globally convex optimization problem. ${ }^{7}$ The resulting density estimate is

$$
\hat{f}(\beta)=\sum_{r=1}^{R} \hat{\theta}^{r} N\left(\beta \mid \mu^{r}, \sigma^{r}\right)
$$

\subsection{Location Scale Model}

In many applications, the econometrician may not have good prior knowledge about the support region where most of the random coefficients $\beta^{r}$ lie. This is particularly true in models where the covariates are high dimensional. There are three approaches to finding a region of support in such settings.

First, the econometrician may use a preliminary estimate of a logit model with fixed coefficients to determine a region of support for the random coefficients. For example, the econometrician may center the grid for the $\beta^{r}$ at the initial logit estimates.

A second, related approach is that the econometrician may experiment with alternative sets of grid points to see how the choice of grid points influences estimation results. A limitation

\footnotetext{
${ }^{7}$ If the true distribution has a continuous density function, using an estimator that imposes that the density estimate is smooth may provide better statistical performance than an estimator that imposes no restrictions on the true distribution function. We do not formally derive the rate of convergence of the estimator for densities or compare the rate to the rate for the earlier estimator for CDFs, which is derived in Section 4.
} 
of this approach is that this introduces a pre-test bias and the standard errors that we present later in the paper will need to be adjusted. For example, a procedure where a researcher uses a diffuse grid with a wide support and then re-estimates the model after increasing the detail of the grid where mass appears to be located is, in its full statistical structure, a two-step estimator. Rigorous standard errors should account for both of the estimation-steps. ${ }^{8}$

A third approach is to introduce location and scale parameters. To illustrate the idea, let the unscaled basis vectors $\left\{\beta^{r}\right\}_{r=1}^{R}$ lie in the set $[0,1]^{K}$, that is, the $K$-fold Cartesian product of the unit interval. We include a set of location and scale parameters $\mu_{k}$ and $\sigma_{k}, k=1, \ldots, K$ and define the $r$ th random coefficient for the $k$ th characteristic as $\mu_{k}+\sigma_{k} \beta_{k}^{r}$.

In numerical optimization, we now search over $R+2 K$ parameters corresponding to $\theta$ and $\mu=\left(\mu_{1}, \ldots, \mu_{K}\right)^{\prime}$ and $\sigma=\left(\sigma_{1}, \ldots, \sigma_{K}\right)^{\prime}$. Market shares predictions for type $r$ are

$$
g_{j}\left(x_{i}, \mu+\sigma \beta^{r}\right)=\frac{\exp \left(\sum_{k=1}^{K} x_{k, i, j}\left(\mu_{k}+\sigma_{k} \beta_{k}^{r}\right)\right)}{1+\sum_{j^{\prime}=1}^{J} \exp \left(\sum_{k=1}^{K} x_{k, i, j^{\prime}}\left(\mu_{k}+\sigma_{k} \beta_{k}^{r}\right)\right)},
$$

where $\sigma \beta^{r}$ is assumed to represent element-by-element multiplication. Our estimator for the weights solves the nonlinear least squares problem

$$
\begin{aligned}
& \min _{\mu, \sigma, \theta} \frac{1}{N J} \sum_{i=1}^{N} \sum_{j=1}^{J}\left(y_{i, j}-\sum_{r=1}^{R} \theta^{r} g_{j}\left(x_{i}, \mu+\sigma \beta^{r}\right)\right)^{2} \\
& \text { subject to (2) and (3). }
\end{aligned}
$$

The appropriate MATLAB routine is lsqnonlin, and there is no theorem that the routine will converge to a global minimum. Note that the model has $2 K$ nonlinear parameters and the remaining $R$ parameters still enter linearly. Also, $\mu$ and $\sigma$ do not enter the constraints, which are still linear. Using exact, rather than numerical derivatives will improve the speed and convergence of the estimator. ${ }^{9}$ The derivatives to our objective function can be expressed in closed form up to any order, at least for the logit example. In numerical experimentation with the logit example,

\footnotetext{
${ }^{8} \mathrm{~A}$ more sophisticated approach would be to choose a massive grid and then add in a penalty function that increases the objective function for values of $\theta$ that assign nonzero weight to a large number of points. Our consistency theorem fits into the sieve framework of Chen and Pouzo (2009a). Their theorems, which we reference in our proof of consistency, explicitly allow for penalty functions and so our estimator is consistent if such a penalty function is used. We will not discuss penalty functions for conciseness.

${ }^{9}$ Automatic differentiation is an option to compute derivatives.
} 
we have found the convergence of the estimator to be robust.

In many applied problems, the number of characteristics $K$ may be large and it may not be practical to estimate a $K$-dimensional nonparametric distribution of random coefficients. Therefore, it may be desirable to only let a subset of the most important characteristics have random coefficients. This is possible by a trivial modification of (7).

\subsection{Choosing $R$ and the $\beta^{r}$ 's}

We have discussed the location and scale model for when the support of $\beta^{r}$ is not known and the $\beta^{r}$ are picked on a grid. The $\beta^{r}$ in this case can be chosen by using a random number generator to make draws from $[0,1]^{K}$. We have also experimented with Halton and Weyl sequences; they tend to improve Monte Carlo performance over evenly-spaced grids. The rate of convergence derived in section 4 uses results from the theory of quadrature. Another rate of convergence, derived in section 4.1, relies on choosing a grid using random draws from a chosen distribution.

It is also natural to ask how to choose $R$, the number of basis components. The nonparametric rates of convergence in section 4 are not useful for choosing $R$ in a finite sample. However, the rates do suggest that $R$ should not increase too quickly with $N$, the number of observations. In Monte Carlo experiments in section 6 , we set $R=\frac{N}{40}$. Our Monte Carlos show a small $R$ (say a few hundred basis vectors) can give a good approximation to $F(\beta)$ in a finite sample.

\subsection{Additional Examples}

As we discussed in the introduction to this section, our estimator can in principal be applied to any setting where the model can be written in the form (1). Below, we discuss some additional examples that fit into in our framework that may be of interest to applied researchers.

\subsubsection{Aggregate Data}

Our estimator can still be used if the researcher has access to data on market shares $s_{j, t}$, rather than individual-level choice data $y_{i, j}$. Index markets by $t=1, \ldots, T$. In this framework, we assume that the utility of person $i$ when the type of $i$ is $r$ is

$$
u_{i, j}^{r}=x_{j, t}^{\prime} \beta^{r}+\epsilon_{i, j}
$$

In this model, the utility of individuals is a function of product- and market-varying attributes

$x_{j, t}$. In applied work, characteristics will vary across markets in the sample. If a market has a 
continuum of consumers, our modeling framework implies that the market share of product $j$ should satisfy

$$
s_{j, t} \approx \sum_{r=1}^{R} \theta^{r} g_{j}\left(x_{t}, \beta^{r}\right)=\sum_{r=1}^{R} \theta^{r} \frac{\exp \left(x_{j, t} \beta^{r}\right)}{1+\sum_{j^{\prime}=1}^{J} \exp \left(x_{j^{\prime}, t} \beta^{r}\right)},
$$

where we let $x_{t}=\left(x_{1, t}^{\prime}, \ldots, x_{J, t}^{\prime}\right)$ denote the stacked vector of the all the $x_{j, t}$ and the $\approx$ sign just indicates that the set $R$ of types is a sieve approximation. Suppose that the economist only observes a noisy measure $\widehat{s}_{j, t}$ of the true share. This is common in applied work. For example, there may be a finite number of consumers in a market. Let the actual share be denoted as $\widehat{s}_{j, t}$. Simple algebra implies that

$$
\widehat{s}_{j, t} \approx\left(\sum_{r=1}^{R} \theta^{r} g_{j}\left(x_{t}, \beta^{r}\right)\right)+\left(\widehat{s}_{j, t}-s_{j, t}\right) .
$$

Under standard assumptions, $E\left[\widehat{s}_{j, t}-s_{j, t} \mid x_{t}\right]=0$. That is, the difference between the measured shares and the true shares is independent of the product characteristics in our model $x_{t}$. This would be the case if the difference between $\widehat{s}_{j, t}$ and $s_{j, t}$ is accounted for by random sampling. Then we can estimate $\theta$ using the regression

$$
\widehat{\theta}=\arg \min _{\theta} \frac{1}{J T} \sum_{t=1}^{T} \sum_{j=1}^{J}\left(\widehat{s}_{j, t}-\sum_{r=1}^{R} \theta^{r} g_{j}\left(x_{t}, \beta^{r}\right)\right)^{2}
$$

subject to (2) and (3).

One advantage of our approach is that it can accommodate measurement error in the market shares. We note that the method of Berry, Levinsohn and Pakes (1995) assumes that $s_{j, t}$ is observed without error by the economist. If the number of persons in a survey or in a market is small, this assumption may be a poor approximation.

A drawback of our approach is that it may require less attractive assumptions than Berry, Levinsohn and Pakes (1995) to account for price endogeneity and aggregate demand shocks. One could use the control function approach of Petrin and Train (2009) to account for this problem. Petrin and Train argue that the control function works well in the applied examples that they consider. However, it has two potential drawbacks. The first is that this approach assumes that there is a reduced-form pricing equation that permits the econometrician to infer the omitted product attributes from the observed prices. In general, it is unknown whether this reduced form exists. The second limitation is that the control function approach requires inserting a generated 
regressor that depends on a nonparametric function into our estimator. This complication is beyond the scope of our asymptotic theory.

\subsubsection{Dynamic Programming Models}

Our approach can be applied to dynamic discrete choice models as in Rust $(1987,1994)$. We generalize the framework he considers by allowing for a nonparametric distribution of random coefficients. Suppose that the flow utility of agent $i$ in a period $t$ from choosing action $j$ is

$$
u_{i, j, t}=x_{i, j, t}^{\prime} \beta_{i}+\epsilon_{i, j, t}
$$

The error term $\epsilon_{i, j, t}$ is a preference shock for agent $i$ 's utility to choice $j$ at time period $t$. For simplicity, the error term is i.i.d. extreme value across agents, choices and time periods. Agent $i$ 's decision problem is dynamic because there is a link between the current and future values of $x_{i, t}=\left(x_{i, 1, t}^{\prime}, \ldots, x_{i, J, t}^{\prime}\right)$ through current decisions. Let $\pi\left(x_{i, t+1} \mid x_{i, t}, j_{i, t}\right)$ denote the transition probability for the state variable $x_{i, t}$ as a function of the action of the agent, $j_{i, t}$. This does not involve random coefficients and we assume that it can be estimated in a first stage.

The goal is to estimate $F(\beta)$, the distribution of the random coefficients. Again we pick $R$ basis vectors $\beta^{r}$. For each of the $R$ basis vectors, we can solve the corresponding singleagent dynamic programming problem for the state $x_{i, t}$ value functions, $V^{r}\left(x_{i, t}\right)$. Once all value functions $V^{r}\left(x_{i, t}\right)$ are known, the choice probabilities $g_{j}\left(x_{i}, \beta^{r}\right)$ for all combinations of choices $j$ and states $x_{i, t}$ can be calculated as

$$
g_{j}\left(x_{i, t}, \beta^{r}\right)=\frac{\exp \left(x_{i, j, t}^{\prime} \beta^{r}+\delta E\left[V^{r}\left(x_{i, t+1}\right) \mid x_{i, t}, j\right]\right)}{\sum_{j^{\prime}=1}^{J} \exp \left(x_{i, j^{\prime}, t}^{\prime} \beta^{r}+\delta E\left[V^{r}\left(x_{i, t+1}\right) \mid x_{i, t}, j^{\prime}\right]\right)}=\frac{\exp \left(v^{r}\left(j, x_{i, t}\right)\right)}{\sum_{j^{\prime}=1}^{J} \exp \left(v^{r}\left(j^{\prime}, x_{i, t}\right)\right)}
$$

where $v^{r}\left(j, x_{i, t}\right)$ is Rust's choice-specific continuation value, here implicitly defined in the above equation. The scalar $\delta \in[0,1)$ is a discount factor fixed by the researcher before estimation, as is usually done in empirical practice. ${ }^{10}$

We use panel data on $N$ panels of length $T$ each. Suppose that we observe $y_{i, j, t}$, an indicator variable equal to one if $j$ is chosen at time $t$ by agent $i$. Let $x_{i, t}$ be the state of agent $i$ at time

\footnotetext{
${ }^{10}$ Alternatively, the discount factor could be a random parameter and the goal would be to estimate the distribution of $(\delta, \beta)$.
} 
$t$. We can then estimate

$$
\widehat{\theta}=\arg \min _{\theta} \frac{1}{N J T} \sum_{i=1}^{N} \sum_{t=1}^{T} \sum_{j=1}^{J}\left(y_{i, j, t}-\sum_{r=1}^{R} \theta^{r} g_{j}\left(x_{i, t}, \beta^{r}\right)\right)^{2}
$$

subject to $(2)$ and $(3)$.

As in the earlier examples, we could use normal distributions as basis functions in order to smooth the estimates of the random coefficients. However, it is not desirable to use the location scale model because modifying these parameters would require us to re-solve the model. The idea of presolving a complex economic model for only $R$ types before optimization commences is also found in Ackerberg (2009), although Ackerberg's approach is parametric instead of nonparametric on the distribution of random coefficients. ${ }^{11}$

It is often the case that the information on a panel can provide more information on heterogeneity than $T$ repeated cross sections. We can explicitly incorporate this use of panel data into our estimator. Let $w$ index a sequence of choices for each time period $t=1, \ldots, T$ called $w_{1}, \ldots, w_{T}$. For example, a choice sequence $w$ could be $w_{1}=5, w_{2}=2, w_{3}=3, \ldots$ If there are $J$ choices per period, there are $W=J^{T}$ sequences that could occur. Let $y_{i, w}$ be equal to 1 if agent $i$ takes action sequence $w$ over the $T$ periods. This minimization problem when panel data is used for extra information on heterogeneity is

$$
\begin{aligned}
& \widehat{\theta}=\arg \min _{\theta} \frac{1}{N W} \sum_{i=1}^{N} \sum_{w=1}^{W}\left(y_{i, w}-\prod_{t=1}^{T}\left(\sum_{r=1}^{R} \theta^{r} g_{w_{t}}\left(x_{i, t}, \beta^{r}\right)\right)\right)^{2} \\
& \text { subject to (2) and (3). }
\end{aligned}
$$

In this case, the estimator matches sequences of choices.

\subsubsection{Joint Discrete and Continuous Demand as in Fox and Gandhi (2009b)}

We can also estimate a model with a joint discrete and continuous choice using our methods. Fox and Gandhi (2009b) introduce identification results for this type of model. Suppose that a consumer purchases a particular type of air conditioner according to the logit model. Conditional on purchase, we observe the electricity consumption of the air conditioner, a measure of usage. Let the notation for the discrete choice be the same as before. The electricity usage equation of

\footnotetext{
${ }^{11}$ Ackerberg cannot estimate his model using linear regression, although an advantage is the ease of allowing homogeneous parameters that enter into a linear index that also has an additive, heterogeneous error.
} 
consumer $i$ of type $r$ for air conditioner $j$ is

$$
a_{i, j}^{r}=w_{i, j}^{\prime} \gamma_{j}^{r}+\eta_{j}^{r}
$$

where $w_{i, j}$ is a vector of observable characteristics that affect electricity demand. There can be overlap between the elements of $x_{i, j}$ and $w_{i, j}$. The parameter $\gamma_{j}^{r}$ is a potentially choice-specific random coefficient vector for type $r$ in the outcome equation. The scalar $\eta_{j}^{r}$ is a choice-specific error term. Let $w_{i}=\left(w_{i, 1}, \ldots, w_{i, J}\right), \gamma=\left(\gamma_{1}, \ldots, \gamma_{J}\right)$ and $\eta=\left(\eta_{1}, \ldots, \eta_{J}\right)$.

Because the dependent variable includes a continuous element, we need to exploit the general model in (1) and work with a set $A=\left[a_{j}^{l}, a_{j}^{l+1}\right)$ for the real-valued dependent variable. The researcher must discretize the continuous outcome variable $a_{j}$ by choosing $L$ bins: $\left[a_{j}^{0}, a_{j}^{1}\right)$, $\left[a_{j}^{1}, a_{j}^{2}\right)$, through $\left[a_{j}^{L-1}, a_{j}^{L}\right)$. A higher $L$ increases the computational burden and the closeness of the approximation to the continuous outcome model.

Let $y_{i, j}^{l}$ be 1 when consumer $i$ purchases air conditioner $j$ and consumes electricity between the lower and upper bounds $a_{j}^{l}$ and $a_{j}^{l+1}$. Then

$$
\begin{aligned}
\operatorname{Pr}\left(y_{i, j}^{l}=1 \mid x_{i}, w_{i} ; \beta^{r}, \gamma^{r}, \eta^{r}\right)= & g_{j, l}\left(x_{i}, w_{i}, \beta^{r}, \gamma^{r}, \eta^{r}\right)= \\
& \frac{\exp \left(x_{i, j}^{\prime} \beta^{r}\right)}{1+\sum_{j^{\prime}=1}^{J} \exp \left(x_{i, j^{\prime}}^{\prime} \beta^{r}\right)} 1\left[a_{j}^{l} \leq w_{i, j}^{\prime} \gamma_{j}^{r}+\eta_{i, j}^{r}<a_{j}^{l+1}\right] .
\end{aligned}
$$

The unknown object of interest is the joint distribution $F(\beta, \gamma, \eta)$ of the multinomial choice random coefficients $\beta$, the electricity usage random coefficients $\gamma$, and the additive errors in utility usage, $\eta$. In this case one can choose a grid of taste parameters $\left\{\beta^{r}, \gamma^{r}, \eta^{r}\right\}_{r=1}^{R}$. A statistical observation is $\left(j_{i}, a_{i, j}, x_{i}, w_{i}\right)$, which can be transformed into $\left(\left\{y_{i, j}^{l}\right\}_{1 \leq j \leq J, 0 \leq l<L}, x_{i}, w_{i}\right)$ by a change of variables. Data on $a_{i, k}$ for $k \neq j_{i}$ is not needed for this transformation. The estimate of $\theta$ minimizes the objective function

$$
\widehat{\theta}=\arg \min _{\theta} \frac{1}{N J} \sum_{i=1}^{N} \sum_{j=1}^{J} \sum_{l=1}^{L}\left(y_{i, j}^{l}-\sum_{r=1}^{R} \theta^{r} g_{j, l}\left(x_{i}, w_{i}, \beta^{r}, \gamma^{r}, \eta^{r}\right)\right)^{2}
$$

subject to (2) and (3),

where $z_{i, j, l}$ is the vector of the $R z_{i, j, l}^{r}$ 's from (10). Estimating $\theta$ provides a nonparametric 
estimator of $F(\beta, \gamma, \eta)$,

$$
\hat{F}(\beta, \gamma, \eta)=\sum_{r=1}^{R} \hat{\theta}^{r} 1\left[\beta^{r} \leq \beta, \gamma^{r} \leq \gamma, \eta^{r} \leq \eta\right] .
$$

This distribution completely determines the model.

This joint continuous and discrete demand model is an example of a selection model. In this example, the continuous outcome $a_{i, j}$ is selected because the researcher only observes the electricity usage for air conditioner $j$ for those individuals who purchase air conditioner $j$, or $j_{i}=j$. Regressing $a_{i, j}$ on $w_{i, j}$ for those individuals who choose $j$ will not consistently estimate $E\left[\gamma_{j}\right]$ if $\gamma_{j}$ is not statistically independent of $\beta$. Those agents who choose air conditioner $j$, $y_{i, j}=1$, will have certain $\beta$ preferences, and the correlation of $\beta$ with $\gamma$ and the correlation of $x$ with $w$ will induce correlation between $w_{i, j}$ and $\gamma_{i}$ in the sample of those who pick a particular air conditioner $j$. However, jointly modeling both the choice of air conditioner and electricity usage removes this selection problem. Note that we allow random coefficients in both the selection (multinomial choice) and outcome (usage) parts of the model. Commonly, selection models focus on allowing random coefficients in only the outcome equation. Again, see Fox and Gandhi (2009b) for identification results for this type of selection model.

\subsubsection{Endogenous Regressors as in Fox and Gandhi (2009a)}

In some cases, the regressors may be correlated with omitted factors. Consider the multinomial choice model. Fox and Gandhi (2009a) address the correlation of $x_{i, j}$ with $\beta_{i}$ using instrumental variables and an auxiliary equation, in which the values of the endogenous regressors are given as a function of exogenous regressors and instruments. For example, if price $a_{i, j}$ is an endogenous regressor and $w_{i, j}$ are the instruments for price, then the auxiliary equation for type $r$ is $a_{i, j}^{r}=$ $w_{i, j}^{\prime} \gamma_{j}^{r}+\eta_{j}^{r}$, which is the same notationally as (9). The difference here is that price $a_{i, j}$ is observed for all $J$ choices for each agent. There is no selection problem. Instead, there is a traditional omitted variables problem where $a_{i, j}$ enters the logit demand model as a component of $x_{i, j}$ and the random variable $a_{i, j}$ is not independent of $\beta_{i}$, the vector of random coefficients in the logit model.

Our estimator can be applied to the model where Fox and Gandhi (2009a) study identification. Estimation works with the reduced form of the model. Let $x_{i, j, 1}=a_{i, j}$, or the endogenous regressor price is the first characteristic in the vector of product characteristics $x_{i, j}$. Let $\beta_{1}$ reflect the random coefficient on price in the discrete choice utility. Let $\tilde{\beta}_{i}$ be the $K-1$ other, 
non-price random coefficients and let $\tilde{x}_{i, j}$ be the $K-1$ other, non-price product characteristics for product $j$. We reuse a lot of the notation from the example of mixed discrete and continuous choice above. With the borrowed notation,

$$
\begin{aligned}
& \operatorname{Pr}\left(y_{i, j}^{l}=1 \mid x_{i}, w_{i} ; \beta^{r}, \gamma^{r}, \eta^{r}\right)=g_{j, l}\left(x_{i}, w_{i}, \beta^{r}, \gamma^{r}, \eta^{r}\right)= \\
& \frac{\exp \left(\tilde{x}_{i, j}^{\prime} \tilde{\beta}^{r}+\beta_{1}^{r}\left(w_{i, j}^{\prime} \gamma_{j}^{r}+\eta_{j}^{r}\right)\right)}{1+\sum_{j^{\prime}=1}^{J} \exp \left(\tilde{x}_{i, j^{\prime}}^{\prime} \tilde{\beta}^{r}+\beta_{1}^{r}\left(w_{i, j^{\prime}}^{\prime} \gamma_{j^{\prime}}^{r}+\eta_{j^{\prime}}^{r}\right)\right)} 1\left[a_{j}^{l} \leq w_{i, j}^{\prime} \gamma_{j}^{r}+\eta_{i, j}^{r}<a_{j}^{l+1}\right] .
\end{aligned}
$$

The key idea here is that we work with the reduced form of the model: we replace the actual $J$ prices in the data $a_{i, j}$ with the $J$ predicted prices $w_{i, j}^{\prime} \gamma_{j}^{r}+\eta_{i, j}^{r}$ from the auxiliary pricing equation. The actual data $a_{i, j}$ that are allowed to be statistically dependent with $\beta_{i}$ do not appear in (13). Only data on $\tilde{x}_{i, j}$, the exogenous regressors, and $w_{i, j}$, the instruments that enter the pricing

equation, are used. We assume $\left(\tilde{x}_{i}, w_{i}\right)$, where $\tilde{x}_{i}=\left(\tilde{x}_{i, 1}^{\prime}, \ldots, \tilde{x}_{i, J}^{\prime}\right)$ and $w_{i}=\left(w_{i, 1}, \ldots, w_{i, J}\right)$, is independent of $\left(\beta_{i}, \gamma_{i}, \eta_{i}\right)$, the heterogeneity realizations.

Estimation proceeds as in (11), except $g_{j, l}\left(x_{i}, w_{i}, \beta^{r}, \gamma^{r}, \eta^{r}\right)$ is now defined by (13). Like in the selection example, the omitted variables example has as its object of interest $F(\beta, \gamma, \eta)$. The estimator of $F(\beta, \gamma, \eta)$ is (12), with the estimates $\hat{\theta}$ coming from the omitted variable bias and not the selection model. Note that the framework of Fox and Gandhi (2009a) allows random coefficients both in the pricing and discrete choice decisions, and those random coefficients have an unrestricted joint distribution $F(\beta, \gamma, \eta)$.

\subsubsection{Endogenous Regressors as in Berry and Haile (2008)}

Berry and Haile (2008) investigate a multinomial choice model where the utility to each choice $j$ in market $t$ includes an additive $\xi_{j, t}$ term, perhaps reflecting an unobserved product characteristic. The endogeneity problem arises from the correlation of price with $\xi_{j, t}$. The authors use a "special regressor" $x_{i, j, K}$ that varies within markets, while the endogenous regressor price varies at the market level. The coefficient on the special regressor $x_{i, j, K}$ has the same sign for all consumers.

Berry and Haile propose a three-step identification strategy. In step 1, the special regressor is used to trace out the marginal CDF for the utility for each of the $J$ choices in each market. The key idea is that the random disturbance $\xi_{j, t}$ that causes the endogeneity problem is the same for all agents in the same market, while the special regressor varies across agents in the same market. With this step, the authors can compute the mean payoff across consumers in the same market for each choice. In step 2, the mean utility of each choice in each market is 
nonparametrically regressed on product characteristics, using instruments. An observation in this second stage is a product in a particular market, not an individual consumer. The error term in the nonparametric regression is $\xi_{j, t}$. As the mean utility value is a continuous variable, the value of $\xi_{j, t}$ is recovered. In the third stage, $\xi_{j, t}$ is an included product characteristic and normal demand estimation can commence. In other words, $\xi_{j, t}$ can be added to the vector $x_{j, t}$ and our standard discrete-choice estimator can be used.

\subsubsection{Endogenous Regressors as in Petrin and Train (2008)}

We mentioned Petrin and Train (2008) above in the discussion of aggregate data. Their method can also be used with individual data. Like Fox and Gandhi (2009a), they specify an auxiliary pricing equation. However, there are no random coefficients in the pricing equation $\left(\gamma_{i}=\gamma \forall i\right)$ and the error term in the pricing equation $a_{i, j}=w_{i, j}^{\prime} \gamma+\eta_{i, j}$ is labeled as an omitted product characteristic. In a first stage, an OLS regression of $a$ on the instrument vector $w$ produces consistent estimates of the error terms $\eta_{i, j}$. These error terms $\eta_{i, j}$ are then treated as observed and then included in $x_{i, j}$, the vector of product characteristic. All endogeneity problems are assumed to arise from not observing $\eta_{i, j}$, so measuring it in a first stage and including it as an observed product characteristic solves the endogeneity problem.

The Petrin and Train (2008) approach is computationally simple and indeed the model is a special case of the model in Fox and Gandhi (2009a), as it does not allow random coefficients in the first stage. Both methods require the assumption of an auxiliary equation that gives the value of the endogenous regressors as a function of instruments. Berry and Haile (2008) avoid using an auxiliary equation by assuming that the endogeneity problem arises from a variable that is constant for all agents within the same market. Berry and Haile can trace out the marginal utility of each choice, holding the variable causing endogeneity problems constant, using a special regressor that varies across agents within the same market.

\section{Consistency for the Distribution in Function Space}

In this section, we assume that the true distribution function $F_{0}$ lies in the space $\mathcal{F}$ of distribution functions on a parameter space $\mathcal{B}$. Let $R(N)$ be the number of grid points chosen with $N$ observations. We wish to show that the estimated distribution function $\hat{F}_{N}(\beta)=\sum_{r=1}^{R(N)} \hat{\theta}^{r} 1\left[\beta^{r} \leq \beta\right]$ converges to the true $F_{0} \in \mathcal{F}$. To prove consistency, we use the recent results for sieve estimators developed by Chen and Pouzo (2009a), hereafter referred to as CP. We view each set of $R$ points 
as a sieve space

$$
\mathcal{F}_{R}=\left\{F \mid \exists \theta^{1}, \ldots, \theta^{R}, \theta^{r} \geq 0, \sum_{r=1}^{R} \theta^{r}=1 \text { s.t. } F(\beta)=\sum_{r=1}^{R} \theta^{r} 1\left[\beta^{r} \leq \beta\right]\right\},
$$

for some fixed choice of grid $\mathcal{B}^{R}=\left\{\beta^{1}, \ldots, \beta^{R}\right\}$. We require $\mathcal{B}^{R} \subseteq \mathcal{B}^{S}$ for $S>R$. Note that this sieve space automatically imposes that the estimated function is a cumulative distribution function. CP suggest other bases for unknown functions; we prefer our choice of sieve spaces in the interests of computational simplicity and the ease of constraining the estimated function to be a probability distribution function over Borel measurable sets.

Based on $\mathrm{CP}$, one can prove that the estimator $\hat{F}_{N}$ converges to the true $F_{0}$ in function space, i.e. in the space of distributions. CP study sieve minimum distance estimators that involve a two-stage procedure. Our estimator is a one-stage sieve least squares estimator (Chen, 2007) and we show its consistency based on CP's general consistency theorem, their Lemma B.1.

Let $y$ be the dependent variable, which represents some discrete outcome. We suppress the notation $A$ for the outcome. We define our sample criterion function as

$$
\hat{Q}_{N}(F) \equiv \frac{1}{N} \sum_{i=1}^{N}\left(y_{i}-\sum_{r=1}^{R} \theta^{r} g\left(x_{i}, \beta^{r}\right)\right)^{2}, F \in \mathcal{F}_{R}
$$

We minimize over the unknown weights $\theta^{r}$ and subject to the constraints $\theta^{r} \geq 0 \forall r$ and $\sum_{r=1}^{R(N)} \theta^{r}=1$. One can add to the least squares objective function a function that penalizes values of $F$ that are less smooth, but we prove consistency using only the properties of the sieve space, not the penalization function. See CP for more on optional penalization functions.

We must choose a metric to show the consistency of $\hat{F}_{N}$ for $F_{0}$. We choose the Lévy-Prokhorov metric, denoted by $d_{\mathrm{LP}}(\cdot)$, a metrization of the weak topology for the space of multivariate distributions. The Lévy-Prokhorov metric in the space of $\mathcal{F}$ is defined on a metric space $(\mathcal{B}, d)$.

We say two distributions $F_{1}$ and $F_{2}$ are distinguishable in terms of the Lévy-Prokhorov metric if $d_{\mathrm{LP}}\left(F_{1}, F_{2}\right)=0$ implies $F_{1}=F_{2} \cdot{ }^{12}$ The space $\mathcal{F}$ of distributions on $\mathcal{B}$ is compact in the weak topology if $\mathcal{B}$ itself is compact (Parthasarathy 1967, Theorem 6.4). We do not formally use compactness of the true function space $\mathcal{F}$ in our proof of consistency. It is possible that compactness rules out the ill-posed inverse problem that CP investigate and may be present in the heterogeneity problem under other metrics. We require that the grid of points be chosen so

\footnotetext{
${ }^{12}$ See Zolotarev (2001) for a formal definition of the Lévy-Prokhorov metric.
} 
that the grid $\mathcal{B}^{R(N)}$ becomes dense in $\mathcal{B}$. The theorem is one of consistency and so does not offer any additional insight on how choices of grids relate to statistical performance.

\section{Theorem 3.1}

- Let $\mathbf{F}$ be the space of all distribution functions on a finite-dimensional real space $\mathcal{B}$, where $\mathcal{B}$ is compact. Further let $\mathcal{F} \subseteq \mathbf{F}$ be the space of distributions distinguishable in terms of the the Lévy-Prokhorov metric. We assume the true $F_{0} \in \mathcal{F}$.

- Let $\mathcal{B}^{R(N)}$ become dense in $\mathcal{B}$ as $N \rightarrow \infty$ where $\frac{R(N) \log R(N)}{\sqrt{N}} \rightarrow 0$. Let $\left\{y_{i}, x_{i}\right\}_{i=1}^{N}$ be i.i.d.

- Let the space of $x, \mathcal{X}$, be compact and let any open ball in $\mathcal{X}$ have positive measure.

- Let each $g(x, \beta)$ be continuous and bounded in both $x$ and $\beta$.

- Assume the model $\{g(x, \beta) \mid \beta \in \mathcal{B}\}$ is identified with positive probability, meaning $F_{0}$ and any $F_{1} \neq F_{0}, F_{1} \in \mathcal{F}$ give $P_{0}(x) \neq P_{1}(x)$ for $x \in \tilde{\mathcal{X}}$, where $\tilde{\mathcal{X}} \subseteq \mathcal{X}$ is a set with positive probability.

- The $R(N) \times R(N)$ matrix $\left(E\left[g\left(X, \beta^{r}\right) g\left(X, \beta^{r^{\prime}}\right)\right]\right)_{1 \leq r, r^{\prime} \leq R(N)}$ is positive definite and its smallest eigenvalue is bounded away from zero uniformly in $R(N)$.

Then $d_{\mathrm{LP}}\left(\hat{F}_{N}, F_{0}\right) \longrightarrow o_{P}(1)$, where $d_{\mathrm{LP}}(\cdot)$ represents the Lévy-Prokhorov metric.

\subsection{Comments on Identification}

We assume that the model is identified in order to focus on this paper's contribution to estimation. In Bajari, Fox, Kim and Ryan (2009), we prove that our lead example, the random coefficients logit, is nonparametrically identified. Our proof relies on the linear index $x^{\prime} \beta$ and the differentiability of $g(x, \beta)$ in $\beta$. Our approach can be adapted to other economic models satisfying these restrictions.

Fox and Gandhi (2009a, 2009b) provide a general framework for proving identification in a class of economic models. They develop conditions on the behavior of the heterogeneous economic agents at different choice situations. These conditions are sufficient but not necessary for identification. The conditions are easier to verify than the "linear independence" condition emphasized in the statistics literature (Teicher 1963). Indeed, stating that a model is "linearly independent" is only a small step from stating that the model is identified. 
We have discussed Berry and Haile's (2008) contribution to discrete choice analysis with unobserved product characteristics previously. Ichimura and Thompson (1997) study binary choice with exogenous characteristics and use the Cramér-Wold (1936) theorem for identification. Gautier and Kitamura (2008) present alternative arguments that give equivalent results. Hoderlein, Klemelä, and Mammen (2008) study the identification and estimation of the density of random coefficients in the linear regression model. They allow endogenous regressors using auxiliary equations (without random coefficients) for the endogenous regressors. Chiappori and Komunjer (2009) recently investigate identification in the multinomial choice model.

\section{Asymptotic Bounds}

We derive asymptotic bounds both for the approximation of conditional choice probabilities, $\operatorname{Pr}\left(y_{i, j}=1 \mid x_{i}\right)$, and for the approximation of the underlying distribution of random coefficients $F(\beta)$ obtained from the estimation of $\theta$ in Section 2 .

Here we explicitly account for a number of $J$ product choices or choice probabilities. To simplify our notation, let $X_{i}=\left(X_{1, i}^{\prime}, \ldots, X_{J, i}^{\prime}\right)$ be the random variable that is the characteristics of all $J$ choices and denote by $\mathcal{X} \equiv \mathcal{X}_{1} \times \ldots \times \mathcal{X}_{J}$ the support of the distribution of $X_{i}$. Here we focus on the logit but the asymptotic theory we develop in this section can be applied to other cases as long as equivalents of Assumption 4.1 and 4.2 below hold. Our results in this section relate to those in Newey (1997) and Chen (2007), although the Riemann and quadrature arguments are more novel.

We maintain the assumption of the nonparametric random utility model with logit errors and consider only distributions with locally integrable densities. The conditional choice probability for alternative $j$ is

$$
P\left(j \mid x_{i}, f\right)=\int\left(\frac{\exp \left(x_{j, i}^{\prime} \beta\right)}{1+\sum_{j^{\prime}=1}^{J} \exp \left(x_{j^{\prime}, i}^{\prime} \beta\right)}\right) f(\beta) d \beta \text { for any } x_{i}=\left(x_{1, i}^{\prime}, \ldots, x_{J, i}^{\prime}\right) \in \mathcal{X},
$$

where $f$ is a density of the random coefficients and the true conditional choice probability is denoted by $P\left(j \mid x_{i}, f_{0}\right)$. We further let the true choice probabilities in the data be $\operatorname{Pr}\left(y_{i, j}=1 \mid x_{i}\right) \equiv$ $P\left(j \mid x_{i}\right)=P\left(j \mid x_{i}, f_{0}\right)$.

Then, we have

$$
y_{i, j}=P\left(j \mid x_{i}\right)+e_{j, i} \text { and } E\left[e_{j, i} \mid X_{1}, \ldots, X_{N}\right]=0
$$


by definition of a choice probability for individual choices. For aggregate data such as market shares, we assume

$$
\hat{s}_{j, t}=P\left(j \mid x_{t}\right)+e_{j, t} \text { and } E\left[e_{j, t}=\hat{s}_{j, t}-s_{j, t} \mid X_{1}, \ldots, X_{T}\right]=0
$$

where $s_{j, t}=P\left(j \mid x_{t}\right)$ and $x_{t}=\left(x_{1, t}^{\prime}, \ldots, x_{J, t}^{\prime}\right)$, so $e_{j, t}$ is a pure measurement error as in Section 2.8.1. Here we focus on individual choice problems and the asymptotics we develop in this section also hold for the aggregate data case with slight modification.

We introduce additional simplifying notation. Define a simplex

$$
\Delta_{R(N)}=\left\{\theta=\left(\theta^{1}, \ldots, \theta^{R(N)}\right) \mid \theta^{r} \geq 0, \sum_{r=1}^{R(N)} \theta^{r}=1\right\}
$$

Recall that we require $\theta^{r} \geq 0$ and $\sum_{r=1}^{R(N)} \theta^{r}=1$ as the $\theta^{r}$ 's are type frequencies in our approximation. We approximate $P\left(j \mid x_{i}\right)$ using $\sum_{r=1}^{R} \theta^{r} g_{j}\left(x_{i}, \beta^{r}\right)$ and $g_{j}\left(x_{i}, \beta^{r}\right) \equiv \frac{\exp \left(x_{j, i}^{\prime} \beta^{r}\right)}{1+\sum_{j^{\prime}=1}^{J} \exp \left(x_{j^{\prime}, i}^{\prime} \beta^{r}\right)}$. Let the approximation to choice probabilities be

$$
\mu_{\theta}\left(j, x_{i}\right)=\sum_{r=1}^{R(N)} \theta^{r} g_{j}\left(x_{i}, \beta^{r}\right) \text { for } \theta \in \Delta_{R(N)}
$$

The estimated weights are

$$
\widehat{\theta}=\operatorname{argmin}_{\theta \in \Delta_{R(N)}} \frac{1}{N J} \sum_{i=1}^{N} \sum_{j=1}^{J}\left(y_{i, j}-\mu_{\theta}\left(j, x_{i}\right)\right)^{2}
$$

Let $K$ be the number of random coefficients. Then we define the parameter space for choice $j$ as the collection of market shares or conditional choice probabilities,

$\mathcal{H}^{(j)}=\left\{P\left(j \mid x_{i}, f\right)\left|M \geq \max _{s=0, \ldots, s} \sup _{\beta \in \mathcal{B}}\right| D^{s} f(\beta) \mid, \int_{\mathcal{B}} f(\beta) d \beta=1, \mathcal{B}=\prod_{k=1}^{K}\left[\underline{\beta}_{(k)}, \bar{\beta}_{(k)}\right], f \in C^{\bar{s}}[\mathcal{B}]\right\}$,

where $D^{s}=\frac{\partial^{s}}{\partial \beta_{(1)}^{\alpha_{1}} \ldots \partial \beta_{(K)}^{\alpha_{K}}}, s=\alpha_{1}+\cdots+\alpha_{K}$ with $D^{0} f=f$, giving the collection of all derivatives of order $s$. Also, $C^{\bar{s}}[\mathcal{B}]$ is a space of $\bar{s}$-times continuously differentiable functions defined on $\mathcal{B}$. We assume $P\left(j \mid x_{i}, f_{0}\right) \in \mathcal{H}^{(j)}$ for $j=1, \ldots, J$. Therefore we assume any element of the class of density functions that generates $\mathcal{H}^{(j)}$ is defined on a Cartesian product $\mathcal{B}$, is uniformly 
bounded by $M$, is $\bar{s}$-times continuously differentiable, and its (all own and partial) derivatives are uniformly bounded by $M$. We assume that the true $f_{0}$ is in each $\mathcal{H}^{(j)}$, that there exist the scalars $M$ and $\bar{s}$, and that $\bar{s}$ is indeed an integer. The definition of $\mathcal{H}^{(j)}$ depends on $M$ and $\bar{s} ; \bar{s}$ will show up in our bounds.

We define the space of approximating functions to choice probabilities,

$$
\mathcal{H}_{R(N)}^{(j)}=\left\{\mu_{\theta}\left(j, x_{i}\right) \mid \theta \in \Delta_{R(N)}\right\}
$$

with $R(N)$ tending to infinity as $N \rightarrow \infty$. In order to show that any $P\left(j \mid x_{i}, f\right) \in \mathcal{H}^{(j)}$ can be approximated by an element of $\mathcal{H}_{R(N)}^{(j)}$, we can put an additional structure on $\theta$ such that

$$
\theta^{r}=\frac{c\left(\beta^{r}\right) f\left(\beta^{r}\right)}{\sum_{r=1}^{R} c\left(\beta^{r}\right) f\left(\beta^{r}\right)}, r=1, \ldots, R
$$

for some chosen weights $c\left(\beta^{r}\right)$ 's and where we pick the $R$ grid points $\left\{\beta^{1}, \ldots, \beta^{R}\right\}$ to become dense in $\mathcal{B}$ as $R(N) \rightarrow \infty$. We also require that the grid points accumulate: the set of $R(N)$ grid points contains the $R(N-1)$ grid points. Then, formally, we have $\mathcal{H}^{(j)}=\mathcal{H}_{\infty}^{(j)}$ since any element in $\mathcal{H}_{R(N)}^{(j)}$ is a corresponding Riemann sum (where we let $c\left(\beta^{r}\right)=1 / R$ ) or more generally a quadrature approximation for an integral like (14) such that

$$
\mu_{\theta}\left(j, x_{i}\right)=\sum_{r=1}^{R} \theta^{r} g_{j}\left(x_{i}, \beta^{r}\right)=\frac{1}{\sum_{r=1}^{R} c\left(\beta^{r}\right) f\left(\beta^{r}\right)} \sum_{r=1}^{R} c\left(\beta^{r}\right) f\left(\beta^{r}\right) g_{j}\left(x_{i}, \beta^{r}\right),
$$

and where we expect $\sum_{r=1}^{R} c\left(\beta^{r}\right) f\left(\beta^{r}\right) \underset{R \rightarrow \infty}{\longrightarrow} \int f(\beta) d \beta=1, \sum_{r=1}^{R} c\left(\beta^{r}\right) f\left(\beta^{r}\right) g_{j}\left(x_{i}, \beta^{r}\right) \underset{R \rightarrow \infty}{\longrightarrow}$ $P\left(j \mid x_{i}, f\right)$, and so $\mu_{\theta}\left(j, x_{i}\right) \underset{R \rightarrow \infty}{\longrightarrow} P\left(j \mid x_{i}, f\right)$. We, therefore, can approximate the observed choice probabilities $P\left(j \mid x_{i}\right)$ arbitrarily well using the logit functions $g_{j}\left(x_{i}, \beta^{r}\right)$.

In what follows, even though our notation is specific to the logit example, we develop the asymptotic theory for general basis functions that satisfy a set of conditions. We let $\|h\|_{L_{2, N}}=$ $\sqrt{\frac{1}{N} \sum_{i=1}^{N} h^{2}\left(x_{i}\right)},\|h\|_{L_{2}}=\sqrt{\int_{\mathcal{X}} h^{2}(x) d \varpi(x)}$ (the norm in $L_{2}$ ), and $\|h\|_{\infty}=\sup _{x \in \mathcal{X}}|h(x)|$ for any function $h: \mathcal{X} \rightarrow \mathbb{R}$, where $\varpi(\cdot)$ denotes the distribution of $X$.

Assumption 4.1 (i) $\left\{e_{j, i}, \ldots, e_{J, i}\right\}$ are independent across $i=1, \ldots, N$; (ii) $E\left[e_{j, i} \mid X_{1}, \ldots, X_{N}\right]=$ 0 for all $j=1, \ldots, J$; (iii) $\left\{X_{1, i}, \ldots, X_{J, i}\right\}$ are $i . i . d$. across $i=1, \ldots, N$; (iv) $\mathcal{X}$ is compact; (v) The density of $X$ is bounded above and is bounded away from zero on $\mathcal{X}$. 
Assumption 4.2 (i) $\left\|P\left(j \mid x_{i}, f_{0}\right)\right\|_{\infty} \leq \zeta_{0}$ and $\left\|g_{j}\left(x_{i}, \beta^{r}\right)\right\|_{\infty} \leq \zeta_{0}$ for some constant $\zeta_{0}>0$ uniformly over $r \leq R(N)$ and for all $j$; (ii) $\left\|g_{j}\left(x_{i}, \beta^{r}\right)\right\|_{L_{2}} \geq c_{0}>0$ uniformly over $r \leq R(N)$ and for all $j$; (iii) the $R(N) \times R(N)$ matrix $\left(E\left[g_{j}\left(X_{i}, \beta^{r}\right) g_{j}\left(X_{i}, \beta^{r^{\prime}}\right)\right]\right)_{1 \leq r, r^{\prime} \leq R(N)}$ is positive definite and its smallest eigenvalue is bounded away from zero uniformly in $R(N)$ and for all $j$.

Assumption 4.1 is about the structure of the data. In Assumption 4.1 (i), we allow for heteroskedasticity of $\left\{e_{j, i}, \ldots, e_{J, i}\right\}$, across different $i$ 's. For the logit case, we satisfy Assumption 4.2 (i) trivially since

$$
g_{j}\left(x_{i}, \beta^{r}\right)=\frac{\exp \left(x_{j}^{\prime} \beta^{r}\right)}{1+\sum_{j^{\prime}=1}^{J} \exp \left(x_{j^{\prime}}^{\prime} \beta^{r}\right)} \leq 1 \text { uniformly over } \beta \text { and } x
$$

and $P\left(j \mid x_{i}, f_{0}\right) \leq 1$ uniformly by construction. In the assumption, the same constant $\zeta_{0}$ applies for all sample sizes $N$. We also satisfy Assumption 4.2 (ii) trivially unless $\mathcal{X}$ has no positive mass. In practice, Assumption 4.2 (iii) requires that the $(R(N) \times R(N))$ matrix $\left(\frac{1}{N} \sum_{i=1}^{N} g_{j}\left(x_{i}, \beta^{r}\right) g_{j}\left(x_{i}, \beta^{r^{\prime}}\right)\right)_{1 \leq r, r^{\prime} \leq R(N)}$ is nonsingular for sufficiently large $N$.

Under these regularity conditions, the bound of the approximation to choice probabilities is obtained by the following theorem.

Theorem 4.1 Suppose Assumptions 4.1 and 4.2 hold. Further suppose $\frac{R(N)^{2} \log R(N)}{N} \rightarrow 0$. Then, we have

$$
\begin{aligned}
& \frac{1}{J} \sum_{j=1}^{J}\left\|\mu_{\widehat{\theta}}\left(j, x_{i}\right)-P\left(j \mid x_{i}, f_{0}\right)\right\|_{L_{2, N}}^{2} \\
= & O_{\mathbb{P}}\left(\frac{R(N) \log (R(N) N)}{N}\right)+O_{\mathbb{P}}\left(\inf _{\mu_{\theta} \in \mathcal{H}_{R(N)}^{(j)}} \frac{1}{J} \sum_{j=1}^{J}\left\|\mu_{\theta}\left(j, x_{i}\right)-P\left(j \mid x_{i}, f_{0}\right)\right\|_{L_{2, N}}^{2}\right) .
\end{aligned}
$$

The condition $\frac{R(N)^{2} \log R(N)}{N} \rightarrow 0$ is required so that the $(R(N) \times R(N))$ matrix

$$
\left(\frac{1}{N} \sum_{i=1}^{N} g_{j}\left(x_{i}, \beta^{r}\right) g_{j}\left(x_{i}, \beta^{r^{\prime}}\right)\right)_{1 \leq r, r^{\prime} \leq R(N)}
$$

is nonsingular with sufficiently large $N$ (see Lemma B.3 in the Appendix). Roughly speaking, in view of asymptotic theory for sieve estimators, Theorem 4.1 tells us that the first term corresponds to an asymptotic variance $(\approx(R(N) / N)$ and the second term corresponds to an 
asymptotic bias term (Chen 2007). To derive convergence rates, we need to obtain the order of bias, i.e., the approximation error rate of our sieve approximation to arbitrary conditional choice probabilities.

Lemma 4.1 For all $x \in \mathcal{X}$ and for any $P\left(j \mid x_{i}, f\right) \in \mathcal{H}^{(j)}$, there exist $\theta^{*} \in \Delta_{R}$ such that

$$
\left|\mu_{\theta^{*}}(j, x)-P(j \mid x, f)\right|=O\left(R^{-\bar{s} / K}\right)
$$

and that $\frac{1}{J} \sum_{j=1}^{J}\left\|\mu_{\theta^{*}}\left(j, x_{i}\right)-P\left(j \mid x_{i}, f\right)\right\|_{L_{2, N}}^{2}=O_{\mathbb{P}}\left(R^{-2 \bar{s} / K}\right)$.

The approximation error rate in Lemma 4.1 shows that we have faster convergence with a smoother true density function and slower convergence with a higher dimensional $\beta$.

Now we consider approximating the true conditional choice probability function. From Theorem 4.1 and Lemma 4.1 , we can find $\theta_{0}^{*} \in \Delta_{R(N)}$ (i.e. $\left.\mu_{\theta_{0}^{*}}(j, \cdot) \in \mathcal{H}_{R(N)}^{(j)}\right)$ and $R(N)$ such that

$$
\frac{1}{J} \sum_{j=1}^{J}\left\|\mu_{\theta_{0}^{*}}\left(j, x_{i}\right)-P\left(j \mid x_{i}, f_{0}\right)\right\|_{L_{N}^{2}}^{2}=O_{\mathbb{P}}\left(R^{-2 \bar{s} / K}\right) \asymp O_{\mathbb{P}}\left(\frac{R(N) \log (R(N) N)}{N}\right) .
$$

With this choice of $R(N)$, one will obtain the optimal convergence rate that balances the bias and variance of the sieve estimator. We conclude:

Theorem 4.2 Suppose Assumptions 4.1 and 4.2 hold. Further suppose $\frac{R(N)^{2} \log R(N)}{N} \rightarrow 0$ and $\bar{s}>K / 2$. Then, we have

$$
\begin{aligned}
\frac{1}{J} \sum_{j=1}^{J}\left\|\mu_{\widehat{\theta}}\left(j, x_{i}\right)-P\left(j \mid x_{i}, f_{0}\right)\right\|_{L_{N}^{2}}^{2} & =O_{\mathbb{P}}\left(\frac{R(N) \log (R(N) N)}{N}\right) \text { and } \\
\sum_{r=1}^{R(N)}\left|\widehat{\theta}^{r}-\theta_{0}^{* r}\right| & =O_{\mathbb{P}}\left(R(N) \sqrt{\frac{\log (R(N) N)}{N}}\right) .
\end{aligned}
$$

One can let $R(N)=C \cdot N^{\rho}$. Combining the rate conditions on $R(N)$, we note that $\rho$ should be slightly larger than $\frac{K}{2 \bar{s}+K}$ (from $(17)$ ) and be smaller than $1 / 2$ (from $\frac{R(N)^{2} \log R(N)}{N} \rightarrow 0$ ). Also note that $\bar{s}>K / 2$ is required to have such a $\rho$ exist.

Theorem 4.2 establishes the bounds of the distance between the true conditional choice probability and the approximated conditional choice probability as well as the $L_{1}$ distance between $\widehat{\theta}$ and $\theta_{0}^{*}$. The $L_{1}$ metric for the discrepancy between $\widehat{\theta}$ and $\theta_{0}^{*}$ is reasonable because $\widehat{\theta}$ and $\theta_{0}^{*}$ are frequency parameters (Devroye and Gyorfi 1985, Chapter I). From these bounds, the convergence rates are trivially obtained. 
We can also estimate the distribution function using our estimates $\widehat{\theta}=\left(\widehat{\theta}^{1}, \ldots, \widehat{\theta}^{R}\right)^{\prime}$. Denote by $F_{0}(\beta)$ the true distribution of $\beta$. Also let $\mathcal{B}_{0}$ be the support (or compact subset of the support) of $F_{0}(\beta)$. One can estimate the distribution of the random coefficients using the following empirical distribution based on our estimates

$$
\widehat{F}_{N}(\beta)=\sum_{r=1}^{R(N)} \widehat{\theta}^{r} 1\left[\beta^{r} \leq \beta\right]
$$

We show that $\widehat{F}_{N}(\beta)$ can approximate the true distribution function $F_{0}(\beta)$. The approximation rate holds pointwise in $\beta$ rather than in a function space.

Theorem 4.3 Suppose Assumptions in Theorem 4.2 hold. Then,

$$
\left|\widehat{F}_{N}(\beta)-F_{0}(\beta)\right|=O_{\mathbb{P}}\left(R(N) \sqrt{\frac{\log (R(N) N)}{N}}\right)+O\left(R(N)^{-\bar{s} / K}\right) \text { a.e. } \beta \in \mathcal{B}_{0} .
$$

The bound is divided into a rate involving the statistical sampling error and one involving the approximation of a true distribution by a finite number of grid points. Again, from this bound, we have faster convergence with a smoother true density function and slower convergence with a higher dimensional $\beta$.

\subsection{Breaking the Dimensionality Problem using Random Grids}

We have obtained the approximation error (bias term) in Lemma 4.1 by exploiting the smoothness of the density function and the logit function where the degree of smoothness breaks the curse of dimensionality. Similarly to the arguments of Rust (1997) for dynamic programming, we can also handle the dimensionality problem using a random grids approach. Suppose the $R$ number of grid points are drawn independently from the multivariate uniform distribution such that

$$
\beta_{(k)}^{r}=\underline{\beta}_{(k)}+\left(\bar{\beta}_{(k)}-\underline{\beta}_{(k)}\right) u_{(k)}^{r}
$$

for $k=1, \ldots, K$ where $u^{r}=\left(u_{(1)}^{r}, \ldots, u_{(K)}^{r}\right)^{\prime}$ follows the multivariate uniform distribution on $[0,1]^{K}$. Further let

$$
\theta^{* r}=\frac{(1 / R) f\left(\beta^{r}\right)}{\sum_{r=1}^{R}(1 / R) f\left(\beta^{r}\right)} .
$$


Then we have

$$
\mu_{\theta^{*}}\left(j, x_{i}\right)=\sum_{r=1}^{R} \theta^{* r} g_{j}\left(x_{i}, \beta^{r}\right)=\frac{1}{(1 / R) \sum_{r=1}^{R} f\left(\beta^{r}\right)} \frac{1}{R} \sum_{r=1}^{R} f\left(\beta^{r}\right) g_{j}\left(x_{i}, \beta^{r}\right) .
$$

Note that applying the law of large numbers,

$$
\begin{aligned}
\frac{1}{R} \sum_{r=1}^{R} f\left(\beta^{r}\right) g_{j}\left(x_{i}, \beta^{r}\right) \underset{R \rightarrow \infty}{\longrightarrow} \int_{[0,1]^{K}} f(\underline{\beta}+(\bar{\beta}-\underline{\beta}) u) \frac{\exp \left(x_{j, i}^{\prime}(\underline{\beta}+(\bar{\beta}-\underline{\beta}) u)\right)}{1+\sum_{j^{\prime}=1}^{J} \exp \left(x_{j^{\prime}, i}^{\prime}(\underline{\beta}+(\bar{\beta}-\underline{\beta}) u)\right)} d u \\
\quad=\frac{1}{\prod_{k=1}^{K}\left(\bar{\beta}_{(k)}-\underline{\beta}_{(k)}\right)} \int_{\mathcal{B}} f(\beta) \frac{\exp \left(x_{j, i}^{\prime} \beta\right)}{1+\sum_{j^{\prime}=1}^{J} \exp \left(x_{j^{\prime}, i}^{\prime} \beta\right)} d \beta,
\end{aligned}
$$

where the equality holds by applying the change of variables in the integral. Similarly we have

$$
(1 / R) \sum_{r=1}^{R} f\left(\beta^{r}\right) \underset{R \rightarrow \infty}{\longrightarrow} \frac{1}{\prod_{k=1}^{K}\left(\bar{\beta}_{(k)}-\underline{\beta}_{(k)}\right)} \int_{\mathcal{B}} f(\beta) d \beta=\frac{1}{\prod_{k=1}^{K}\left(\bar{\beta}_{(k)}-\underline{\beta}_{(k)}\right)} .
$$

Applying above two results to (19), we conclude

$$
\mu_{\theta^{*}}\left(j, x_{i}\right) \underset{R \rightarrow \infty}{\longrightarrow} \int_{\mathcal{B}} f(\beta) \frac{\exp \left(x_{j, i}^{\prime} \beta\right)}{1+\sum_{j^{\prime}=1}^{J} \exp \left(x_{j^{\prime}, i}^{\prime} \beta\right)} d \beta=P\left(j \mid x_{i}, f\right)
$$

where we pick $\theta^{*}$ as in (18). Finally we obtain the approximation error rate (bias term), applying the Lindeberg-Levy central limit theorem and the law of large numbers.

Lemma 4.2 For all $x \in \mathcal{X}$ and for any $P(j \mid x, f) \in \mathcal{H}^{(j)}$, with $\theta^{*}$ as in (18), we have that

$$
\left|\mu_{\theta^{*}}(j, x)-P(j \mid x, f)\right|=O_{\mathbb{P}}\left(R^{-1 / 2}\right) .
$$

and that $\frac{1}{J} \sum_{j=1}^{J}\left\|\mu_{\theta^{*}}(j, x)-P(j \mid x, f)\right\|_{L_{2, N}}^{2}=O_{\mathbb{P}}\left(R^{-1}\right)$.

The key conclusion is that these rates do not depend on $K$, the number of random coefficients. We can replace Lemma 4.1 with Lemma 4.2 and obtain alternative bounds for the conditional choice probability and the distribution of random coefficients. 


\section{$5 \quad$ Estimating Confidence Regions with Finite Types}

The literature on sieve estimation (Chen and Pouzo 2009a) does not have asymptotic distribution theory for the infinite-dimensional unknown parameter, $F(\beta)$. Given the state of the literature, we focus on computing standard errors using a parametric method, which requires the assumption that the set of $R$ types used in estimation is the true set of $R$ types that generates the data. Under this assumption, one can use the common OLS heteroskedasticity-consistent standard errors for the unknown weights, $\theta^{1}, \ldots, \theta^{R}$. The traditional OLS confidence intervals are computed using the unconstrained point estimates in section 2.3 instead of the point estimates with constraints from section 2.4. However, the confidence regions so constructed give correct coverage (more than $95 \%$ ) for both the estimates with and without the constraints (Andrews and Guggenberger 2009b, footnote 7). We use the common standard error formulas in our empirical example, below.

One needs to use heteroskedasticity-consistent standard errors because the errors in a linear probability model such as (6) are heteroskedastic. One also should cluster the standard errors at the level of the "regression observations" $j=1, \ldots, J$ for each statistical observation $i=1, \ldots, N$. Recall that for each $i$, there are $J$ "regression observations" in (6) because each inside good $j$ has a separate term in the least-squares objective function. After constructing the OLS confidence intervals for $\hat{\theta}^{r}$, one should remove infeasible values by reporting, for a $95 \%$, two-sided confidence interval,

$$
[0,1] \cap\left[\hat{\theta}^{r}-1.96 \cdot \mathrm{SE}\left(\hat{\theta}^{r}\right), \hat{\theta}^{r}+1.96 \cdot \mathrm{SE}\left(\hat{\theta}^{r}\right)\right]
$$

where $\mathrm{SE}\left(\hat{\theta}^{r}\right)$ is the standard error adjusted for heteroskedasticity and clustered across the $J$ "regression observations" for each statistical observation $i$.

Researchers are often not directly interested in confidence intervals for the weights $\theta$ but rather for functions $m(\theta ; X)$, where $X$ is some arbitrary data. For example, researchers may wish to construct confidence intervals for the distribution $\hat{F}(\beta)=m(\hat{\theta} ; X)=\sum_{r=1}^{R} \hat{\theta}^{r} 1\left[\beta^{r} \leq \beta\right]$ evaluated at a particular value of $\beta$ ( $X$ does not enter $m$ here). To construct standard errors for $m(\hat{\theta} ; X)$, one first constructs the distribution of $\widehat{\theta}^{r}-\theta_{0}^{r}$ as above and then uses the delta method. A $95 \%$ confidence interval is then

$$
\begin{aligned}
& {\left[\min _{\theta} m(\theta ; X), \max _{\theta} m(\theta ; X)\right] \cap} \\
& \quad[m(\hat{\theta} ; X)-1.96 \cdot \operatorname{SE}(m(\hat{\theta} ; X)), m(\hat{\theta} ; X)+1.96 \cdot \operatorname{SE}(m(\hat{\theta} ; X))] .
\end{aligned}
$$

Here the minimum and maximum are taking over the values of $\theta$ that satisfy $(2)$ and (3). This is a 
compact set, so the minimum and maximum are obtained. In many examples, it will be possible to deduce the feasible upper and lower bounds for $m(\theta ; X)$ without resorting to computation.

The common heteroskedasticity-consistent standard errors give more than $95 \%$ coverage but, based on our Monte Carlo evidence, are often quite conservative in that the coverage is much more than $95 \%{ }^{13}$ In empirical work, we often find that many of the included $R$ types have estimated weights of $\hat{\theta}^{r}=0$. Thus, in principle one can construct less conservative confidence intervals by recognizing that the parameters on the boundary of the parameter space cannot have an asymptotically normal distribution. ${ }^{14}$ While Andrews (1999, 2002) and Andrews and Guggenberger (2009b) study related cases, the reality is that this recent literature has not developed general-enough results that could allow us to estimate confidence intervals for our problem in a way that gives asymptotically correct coverage as defined by Andrews and Guggenberger. Indeed, Andrews and Guggenberger study only the case of a regression with one inequality constraint and i.i.d. observations. Traditional OLS confidence intervals using fixed-critical values and based on the point estimates imposing the one constraint are recommended by those authors, but there is no suggestion that traditional OLS methods with fixed critical values and based on the point estimates imposing the constraints give asymptotically correct coverage if there are two or more inequality constraints, as in our estimator.

Resampling procedures are a possibility, but one that Andrews and Guggenberger do not currently recommend when a true parameter may lie on a boundary. Andrews (2000) shows that the standard bootstrap is inconsistent but that subsampling and the $m$-out-of- $n$ bootstrap are pointwise consistent. Andrews and Guggenberger (2010) show that the latter two resampling procedures are not uniformly consistent and may have poor finite-sample coverage. Andrews and Guggenberger (2009a, 2009b) discuss a hybrid method where the maximum of a traditional critical value for a $t$-statistic and a subsampled critical value is used to construct confidence intervals for $\theta$. The hybrid subsampling method has correct asymptotic coverage under the definition of Andrews and Guggenberger for the special case of one inequality constraint, but, as Andrews and Guggenberger (2009b) show, so does one of its ingredients, the traditional fixed critical value method that Andrews and Guggenberger recommend. Subsampling by itself does not have correct asymptotic coverage. ${ }^{15}$

\footnotetext{
${ }^{13}$ We performed Monte Carlo studies of using Tikhonov regularization / ridge regression (and Golub, Heath and Wahba's (1979) generalized cross validation method to pick the perturbation value) to compute standard errors. Tikhonov regularization reduced the size of the confidence regions some but the coverage was still much more than $95 \%$.

${ }^{14}$ Statistical inference for linear regression subject to a set of inequality constraints has been studied by Judge and Takayama (1966), Liew (1976), Geweke (1986), and Wolak (1987).

${ }^{15}$ In an unreported Monte Carlo study, we also found that subsampling could undercover the true parameters:
} 
In an appendix available upon request, we show consistency and derive the sampling distribution of the inequality-constrained nonlinear least squares estimator of the location and scale model. The nonlinear distribution also applies if some parameters in the model are homogeneous. As the sampling distribution is derived, we have verified the only regularity condition needed for the pointwise consistency of subsampling (Politis, Romano and Wolf 1999).

\section{Monte Carlo Experiments}

We conduct a Monte Carlo experiment in order to study the finite sample properties of our estimator. We suppose that the true data generating process is indeed a random coefficients logit. In our Monte Carlo study, $x_{j}$ is a $2 \times 1$ vector. We generate $x_{j, 1}$ using the distribution $x_{j, 1} \sim N(1,1)$. Also, $x_{j, 2} \sim N\left(-0.8,0.8^{2}\right)+0.1 x_{j, 1}$. There are $J=10$ products in each of our markets. $J$ does not include the outside option. We use independent normal basis functions in order to approximate a density function rather than a CDF, as section 2.5 discusses. The sample size is $J \cdot T$, the number of products times the number of markets. We use a sample size of 20,000, corresponding to 2000 markets with 10 products each. The number of basis points is $R=\frac{T \cdot J}{40}=500$. There is little measurement error in shares; we calculate market shares for aggregate data generated by 1 million consumers. ${ }^{16}$

Rather than a criterion such as integrated mean squared error, we prefer to test the structural use of our estimates. For each run, after we compute the estimate $\hat{f}(\beta)$, we evaluate its predictive power by drawing new product characteristics (from the same distributions) and predicting shares. We compare our results to those using the true $f^{0}(\beta)$. This tests the structural use of discrete choice models to predict the demand for new goods.

We generate data using three alternative distributions of the random coefficients $f(\beta)$. In the first design, the tastes for characteristics are generated from a mixture of two normals,

$$
0.4 \cdot N\left(\left[\begin{array}{c}
3 \\
-1
\end{array}\right],\left[\begin{array}{cc}
0.2 & -0.1 \\
-0.1 & 0.4
\end{array}\right]\right)+0.6 \cdot N\left(\left[\begin{array}{c}
-1 \\
1
\end{array}\right],\left[\begin{array}{cc}
0.3 & 0.1 \\
0.1 & 0.3
\end{array}\right]\right) \text {. }
$$

All distributions of random coefficients will have a non-trivial variance matrix, while our basis functions are independent distributions of normal random coefficients. In the second design, the

it has coverage less than $95 \%$.

${ }^{16}$ We choose the grid of points using a Halton sequence over the relevant support. We compute numerical integrals using 200 simulated draws. 
true coefficients are generated by a mixture of four normals,

$$
\begin{aligned}
& 0.2 \cdot N\left(\left[\begin{array}{l}
3 \\
0
\end{array}\right],\left[\begin{array}{cc}
0.2 & -0.1 \\
-0.1 & 0.4
\end{array}\right]\right)+0.4 \cdot N\left(\left[\begin{array}{l}
0 \\
3
\end{array}\right],\left[\begin{array}{cc}
0.2 & -0.1 \\
-0.1 & 0.4
\end{array}\right]\right) \\
& +0.3 \cdot N\left(\left[\begin{array}{c}
1 \\
-1
\end{array}\right],\left[\begin{array}{cc}
0.3 & 0.1 \\
0.1 & 0.3
\end{array}\right]\right)+0.1 \cdot N\left(\left[\begin{array}{c}
-1 \\
1
\end{array}\right],\left[\begin{array}{cc}
0.3 & 0.1 \\
0.1 & 0.3
\end{array}\right]\right) .
\end{aligned}
$$

In the third design and final design, the true coefficients are generated by the mixture of six normals,

$$
\begin{aligned}
& 0.1 \cdot N\left(\left[\begin{array}{l}
3 \\
0
\end{array}\right],\left[\begin{array}{cc}
0.2 & -0.1 \\
-0.1 & 0.4
\end{array}\right]\right)+0.2 \cdot N\left(\left[\begin{array}{l}
0 \\
3
\end{array}\right],\left[\begin{array}{cc}
0.2 & -0.1 \\
-0.1 & 0.4
\end{array}\right]\right) \\
& +0.2 \cdot N\left(\left[\begin{array}{c}
1 \\
-1
\end{array}\right],\left[\begin{array}{cc}
0.2 & -0.1 \\
-0.1 & 0.4
\end{array}\right]\right)+0.1 \cdot N\left(\left[\begin{array}{c}
-1 \\
1
\end{array}\right],\left[\begin{array}{ll}
0.3 & 0.1 \\
0.1 & 0.3
\end{array}\right]\right) \\
& +0.3 \cdot N\left(\left[\begin{array}{l}
2 \\
1
\end{array}\right],\left[\begin{array}{cc}
0.3 & 0.1 \\
0.1 & 0.3
\end{array}\right]\right)+0.1 \cdot N\left(\left[\begin{array}{l}
1 \\
2
\end{array}\right],\left[\begin{array}{cc}
0.3 & 0.1 \\
0.1 & 0.3
\end{array}\right]\right) .
\end{aligned}
$$

We summarize our results in Table 1. The first column states the distribution used to generate the random coefficients. The second column is the root mean squared error (RMSE) of our out of sample prediction for market shares. The RMSE is averaged over many new, counterfactual sets of products. It is not averaged over the statistical sampling error in the original estimator; we estimate for each design only once. The final column is the number of basis functions that have positive weight.

The results suggest that our estimator of $f(\beta)$ is excellent for predicting the market shares of new goods, with large samples. ${ }^{17}$ To understand the units, with $J=10$ and a mean share of 0.10 , a prediction error of 0.01 (10\% of 0.1 ) on each product corresponds to a RMSE of $\sqrt{10 \cdot 0.01^{2} / 10}=0.01$. Observed prediction errors are small with this sample size: 0.0001 . Note that we are able to fit the observed market shares well with a fairly small number of basis functions. The mean number of nonzero basis functions ranges from 32 to 99 in the results reported below. More complex true densities require more nonzero basis points for approximation. Even when we make $R$ large, most of these basis functions will have zero probability. This result is consistent with the literature on mixtures, which demonstrates that even quite complicated

\footnotetext{
${ }^{17}$ Although not shown, our estimator is quite good at fitting market share equations with much smaller samples. Larger samples are needed for excellent performance in density estimation, which is not surprising given the curse of dimensionality in estimating an infinite-dimensional object.
} 
distributions can be approximated with a surprisingly small number of mixture components.

We perform 20 replications with different datasets in order to simulate the finite sample properties of our estimator. We use the 20 replications to construct the finite-sample properties of the estimator of the marginal densities of both $\beta_{1}$ and $\beta_{2}$. Figure 1 includes one plot for each of the three designs and each of the two random coefficients. The solid line is the true density as recorded above, while the dashed line is the fitted density for one of the twenty replications. We use the 20 replications to construct the 5th and 95th quantiles of the estimated densities at each point of evaluation. We see that the variability across replications is quite low at this sample size. Our estimator can recover the true marginal densities.

Figure 2 plots the true versus the estimated joint density of $f(\beta)$, for one out of our 20 replications. A visual inspection of the densities shows that we are able to generate excellent fits to the true densities of preferences using our estimator. Our Monte Carlo experiments demonstrate that it is possible to generate good estimates of market shares and the density of random coefficients with a large number of observations. In fact, we get the modes almost spot on, even for the challenging case of six modes.

\section{Empirical Application to Dynamic Programming}

As an illustration of our estimator, we apply it to the dynamic labor supply setting from Duflo, Hanna, and Ryan (2008), hereafter referred to as DHR. They consider the problem of incentivizing teachers to go to work, and estimate a dynamic labor supply model using the method of simulated moments. The model is a single agent, dynamic programming problem with a serially correlated, unobserved state variable. In order to accommodate unobserved heterogeneity across teachers, they estimate a parametric, two-type mixture model. We apply the present estimator to this setting, and show that our approach allows for a more flexible approximation of the underlying heterogeneity. Further, our new estimator is quicker to run and easier to implement.

Teacher absenteeism is a major problem in India, as nearly a quarter of all teachers are absent nationwide on any given day. The absenteeism rate is nearly 50 percent among nonformal education centers, NGO-run schools designed to provide education services to rural and poor communities. To address absenteeism, DHR ran a randomized field experiment where teachers were given a combination of monitoring and financial incentives to attend school. In a sample of 113 rural, single-teacher schools, 57 randomly selected teachers were given a camera and told to take two pictures of themselves with their students on days they attend work. On top of this monitoring incentive, teachers in the treatment group also received a strong financial 
incentive: for every day beyond 10 they worked in a month, they received a 50 rupee bonus on top of their baseline salary of 500 rupees a month. The typical work month in the sample was about 26 days, so teachers that went to school at every opportunity would receive greater wages than the control group, which was paid a flat amount of 1000 rupees per month. The program ran for 21 months, and complete work histories were collected for all teachers over this period.

DHR evaluates this program and find the combination of incentives was successful in reducing absenteeism from 42 percent to 21 percent. In order to disentangle the confounding effects of the monitoring and financial incentives, they exploit nonlinearities in the financial incentive to estimate the labor supply function of the teachers.

A convenient aspect of the intervention is that the financial incentives reset each month. Therefore, the model focuses on the daily work decision of teachers within a month. Denote the number of days it is possible to work in each month as $C$. Let $t$ denote the current day, and let the observed state variable $d$ denote the number of days already worked in the month. Each day a teacher faces a choice of going to school or staying home. The payoff to going to school is zero. The payoff to staying home is equal to $\mu_{i}+\epsilon_{i, t}$, where $\mu_{i}$ is specific to teacher $i$ and $\epsilon_{i, t}$ is a shock to payoffs. The shock $\epsilon_{i, t}$ is serially correlated.

After the end of the month $(C)$, the teachers receive the following payoffs, denominated in rupees, which are a function of how many days that teacher worked in the month:

$$
\pi(d)= \begin{cases}500+(10-d) \cdot 50 & \text { if } d \geq 10 \\ 500 & \text { otherwise }\end{cases}
$$

Let $r$ be the type of the teacher in our approximation. The choice decision facing each teacher in the form of a value function for periods $t<C$ is

$$
V^{r}\left(t, d_{i}, \epsilon_{i, t}\right)=\max \left\{E\left[V^{r}\left(t+1, d_{i}+1, \epsilon_{i, t+1}\right) \mid \epsilon_{i, t}\right], \mu^{r}+\epsilon_{i, t}+E\left[V^{r}\left(t+1, d_{i}, \epsilon_{i, t+1}\right) \mid \epsilon_{i, t}\right]\right\}
$$

At time $C$, the value function simplifies to

$$
V^{r}\left(C, d_{i}, \epsilon_{i, C}\right)=\max \left\{\beta \pi\left(d_{i}+1\right), \mu^{r}+\epsilon_{i, C}+\beta \pi\left(d_{i}\right)\right\}
$$

where $\beta$ is the marginal utility of an additional rupee. There is no continuation value in the right side of $(22)$ as the stock of days worked reset to zero at the end of the month. These value functions illustrate the tradeoff teachers face early in each month between accruing days 
worked, in the hopes of receiving an extra payoff at the end of the month, and obtaining instant gratification by skipping work. ${ }^{18}$

DHR propose a simulated method of moments estimator that matches sequences of days worked at the beginning of each month. They find parameters that generate predicted probabilities for all the possible sequences of work histories in the first five days of each month as close as possible to their empirical counterparts. ${ }^{19}$ This procedure results in $2^{5}-1=31$ linearly independent moments to be matched in each month. They estimate several specifications of the above model using this approach; we focus on their preferred model, in which the shock to the outside option follows an $\operatorname{AR}(1)$ process with correlation parameter $\rho$, and the teacher-specific, deterministic component of the outside option $\mu_{i}$ is drawn from a bimodal normal distribution. Note that this is a very computationally intensive problem, as the researcher must integrate out over both the distribution of outside options $\mu_{i}$ and the serially correlated unobservable $\epsilon_{i, t}$ in order to produce the probabilities of sequences of days worked. This is not a computationally trivial task, as the model requires several hundred thousand simulations in order to produce estimates of the probabilities of working sequences with low variance for each type. Using the parametric mixture, DHR estimate that in a given month 97.6 percent of the teachers have outside options drawn from a normal distribution with mean -0.428 and variance of 0.007 , with the remaining 2.4 percent drawing from a normal distribution with mean 1.781 and variance 0.050 . At the estimated parameters of this model, workers drawing the first distribution generally go to school every day, while workers drawing from second distribution are likely to never attend school during a given month.

There are natural bounds on the level of the outside option, as low values of $\mu_{i}$ lead to teachers always working and high values lead to teachers never working. The autocorrelation parameter is bounded between negative one and positive one. The beta parameter is also sharply bounded, as it has similar effects on work behavior as the outside option outside a narrow range.

We apply the present paper's estimator to this setting, allowing for a nonparametric distribution of heterogeneity in the outside option. We hold the marginal utility of income and

\footnotetext{
${ }^{18}$ The day of the month $t$ and stock of worked days $d$ are naturally discrete state variables. For each combination of $t$ and $d$, the continuous state $\epsilon$ is discretized into 200 bins. For each simulated value of $\epsilon$, the closest bin value is taken to be the actual state for the purposes of dynamic programming. For numerical integration in the calculation of expectations such as $E\left[V^{r}\left(t+1, d_{i}+1, \epsilon_{i, t+1}\right) \mid \epsilon_{i, t}\right]$, the distribution of $\epsilon_{i, t+1}$ is used to calculate the probability $\epsilon_{i, t+1}$ lies in each of the $200 \mathrm{bins}$, and those probabilities weight $V^{r}\left(t+1, d_{i}+1, \epsilon_{i, t+1}\right)$ in the approximation to the expectation.

${ }^{19}$ Considering data on only the first five days in each month both simplifies the computational burden and breaks much of the statistical dependence across the beginning of months (as the correlation of $\epsilon_{i, t}$ across months is low). We treat data from different months as statistically distinct from the viewpoint of the correlation in $\epsilon_{i, t}$.
} 
the autocorrelation parameter at their values estimated under the two-type parametric model estimated in DHR: $\beta=0.013$ and $\rho=0.449 .{ }^{20}$ In other words, we do not estimate those parameters.

We estimate the model with a discrete approximation to the underlying distribution of heterogeneity in the outside option. We let the number of basis functions range between $R=5$ and $R=40$, with the types uniformly distributed across the economic bounds on the outside option. At the lower extreme, $\mu_{i}=-2.5$, the teachers almost always go to work, and at the upper extreme, $\mu_{i}=4.0$, teachers almost never go to work. We solve the model under each of those $R$ draws for every month in the data set. In addition to the intra-month time series variation in the moments, our model is identified from variation in the number of days, the distribution of workdays (teachers receive one day off on the weekends), and the number of holidays, which count as a day worked in the teacher's payoff function, across months. These exogenous sources of variation produce different probabilities of working even under the same set of model primitives.

For each month in the data, we solve the dynamic program for all $R$ types, and then compute the probabilities of all possible sequences of days worked in the first five days of the month. We collate these probabilities together to produce a matrix with $R$ columns and 31 rows, where each row corresponds to the probability of observing a specific work history for that month. We then stack these matrices across months to obtain a large matrix with $R$ columns and $31 * 21=651$ rows corresponding to the $31=2^{5}-1$ possible work histories and the 21 different months. We formed the corresponding vector of empirical probabilities as the vector of dependent variables. We then assigned weights to each of the $R$ types using the inequality constrained OLS estimator. ${ }^{21}$ This estimator is quite similar to the specification in equation (8), except that we do not use panel data to construct sequences of choices for the same teacher across months, only within months.

The estimated distributions of types are shown in Figure 3. We use a discrete-type approximation to the distribution of $\mu_{i}$. The vertical axis is the weight of that type in the probability

\footnotetext{
${ }^{20}$ In a previous draft of the paper, we also experimented with allowing for unobserved heterogeneity in the other two parameters, the marginal utility of income $(\beta)$ and the degree of persistence in the $\operatorname{AR}(1)$ process $(\rho)$, with unrestricted covariance across the parameters. We found that the $\beta$ and $\rho$ parameters were always estimated tightly around their point estimates, and therefore we focus on the distribution of $\mu_{i}$ in what follows.

${ }^{21}$ We use a penalized (for the constraints) Newton's method to minimize the least squares objective function. We use the assumption that the true types are the types included to construct confidence intervals, as in section 5. Confidence intervals are constructed using the standard OLS formulas. An observation is a teacher / month. Five days are used for each teacher / month observation. The number of observations is 1123 teacher / months. The correlation in $\epsilon_{i, t}$ across the first five days should be low, so we do not account for autocorrelation across teacher / months for the same teacher.
} 
mass function that is an approximation to the true distribution of types. The squares represent the point estimates; the sum of these weights is always 1 . The figures also show the $90 \%$ confidence intervals for the weight on each type. The confidence intervals are smaller in the center of the distribution. Keep in mind these are univariate confidence regions for individual weights; the confidence regions for functions of all of the weights, such as $\hat{F}(\beta)$ at a particular $\beta$, may be relatively narrower. In these and subsequent figures, we present four approximations, for $R=5$, $R=10, R=20$, and $R=40$. The $R=40$ estimates suggest a double-peaked distribution in the range a utility of staying home from -0.5 to 0.0 . Three basis points in the range $-0.5-0.0$ are given substantial weight. The right tail is thin: most weights are 0 but the type of $\mu=2.83$ has an estimated frequency of $3 \%$. The middle of this support, -0.25 , gives a value of staying home of $-0.25 / \beta=-0.25 / 0.013=-19$ rupees a day. This means that, at $\epsilon_{i, t}=0$, a modal teacher will go to work for only a standard incentive like the threat of being fired or simply an attitude of professionalism. However, there is a positive probability of teachers with positive values of staying home, $\mu_{i}$. Our nonparametric estimates do not exactly match those from the parametric results in DHR, but they capture the same finding that most teachers are between -0.5 and 0.0 with a small fraction of teachers who always prefer to skip work in the right tail.

Figure 4 shows the fit of the discrete approximation models to the distribution of days worked in the data. Keep in mind we only used the first five days of each month in estimation. The mean predicted distribution matches the observed distribution relatively well. In the specifications with $R>5$, our model tends to underpredict the peak by one day and overpredict the proportion of days worked in the 15-20 range. We note that these fit results are particularly encouraging, as the model does not use the distribution of days worked directly in the estimation; as such, these results are a partial out-of-sample test.

A feature of the teacher data is that there is a completely independent second experiment which was conducted after the first intervention, in which the incentives facing teachers were changed. Instead of working 10 days to get a marginal per-day bonus of 50 rupees, teachers in the second experiment had to work 12 days to get a marginal per-day bonus of 70 rupees. Figure 5 shows the fits of the discrete approximation models in the out-of-sample test. These data are a bit noisier than the original data set, due to a smaller sample size, but the nonparametric model also does a fairly good job of matching the distribution of days worked. Our approach tends to underpredict the proportion of zero days worked and overpredict the number of days worked above 18 .

While the advantage of a nonparametric approach is clear, it is also worth emphasizing that the computational burden of the present estimator is much lower than the parametric alternative. 
Even with good starting values, the parametric approach requires several thousand evaluations of the objective function in a typical Newton-based optimizer. For each guess of the parameters, the dynamic model requires several million forward simulations to produce reliable estimates of the predicted probabilities of work histories, which is a significant computational burden. Furthermore, specification testing in the parametric environment is costly, and the parameter estimates under one type were very different than under two types. One clear benefit of the present approach is that one is able to run literally dozens of different models in less time than it takes to optimize a single parametric specification.

\section{Conclusion}

In this paper, we have proposed a new method for estimating general mixtures model. Our method allows the researcher to drop standard parametric assumptions, such as independent normal random coefficients, that are commonly used in applied work. In terms of computer programming and execution time, our linear regression estimator is easier to work with than simulated likelihood or method of moments estimators for parametric models. Convergence of an optimization routine to the global optimum is guaranteed under linear regression with linear constraints, something that cannot be said for other statistical objective functions. Also, our estimator is much easier to program and to use than alternatives such as the EM algorithm.

Our estimator is useful for estimating a wide variety of models. For example, in a dynamic programming setting, the estimator allows for a nonparametric distribution of random coefficients while simultaneously cutting the computational time compared to the no-random coefficients model. The computational savings arise because we must solve the dynamic program only once for each basis vector.

We explored the asymptotic properties of the linear regression estimator. We showed consistency in the function space of all distributions by viewing our estimator as a sieve estimator and applying techniques in Chen and Pouzo (2009a). Under additional assumptions, we derive the pointwise rate of convergence of the estimated distribution function to the true distribution function. Many alternative mixtures estimators lack asymptotic results in such generality.

We apply our estimator in an empirical example of estimating the distribution of agent preferences in a dynamic programming model with an unobserved, serially correlated state variable. Our estimator use dramatically less programming and execution time than a parametric alternative. 


\section{A Proof of Consistency: Theorem 3.1}

There are two cases. If the true distribution $F_{0} \in \mathcal{F}_{R(N)}$ is in some sieve space, then the estimator becomes parametric after some $R(N)$, and the estimator is consistent. The remainder of the argument assumes $F_{0}$ is not in any sieve space.

We resort to CP's Lemma B.1 in our consistency proof and verify the conditions of CP's Lemma B.1. To provide completeness to readers, we first present our simplified version of CP's Lemma B.1 and its proof.

Lemma A.1 Lemma B.1 of CP: Let $\hat{F}_{N}$ be such that $\hat{Q}_{N}\left(\hat{F}_{N}\right) \leq \inf _{F \in \mathcal{F}_{R(N)}} \hat{Q}_{N}(F)+O_{P}\left(\eta_{N}\right)$ with $\eta_{N}=o(1)$. Suppose the following conditions (B.1.1)-(B.1.4) hold:

- (B.1.1) (i) $Q\left(F_{0}\right)<\infty$; (ii) there is a positive function $\delta(N, R(N)$, $\varepsilon)$ such that

$$
\inf _{F \in \mathcal{F}_{R(N)}: d_{\mathrm{LP}}\left(F, F_{0}\right) \geq \varepsilon} Q(F)-Q\left(F_{0}\right) \geq \delta(N, R(N), \varepsilon)
$$

for each $N \geq 1, R \geq 1, \varepsilon>0$, and $\liminf _{N \rightarrow \infty} \delta(N, R(N), \varepsilon) \geq 0$ for all $\varepsilon>0$.

- (B.1.2) (i) $\mathcal{F} \subseteq \mathbf{F}$ and $\left(\mathbf{F}, d_{\mathrm{LP}}(\cdot)\right)$ is a metric space; (ii) $\mathcal{F}_{R} \subseteq \mathcal{F}_{R+1} \subseteq \mathcal{F}$ for all $R \geq 1$, and there exists a sequence $\Pi_{N} F_{0} \in \mathcal{F}_{R(N)}$ such that $d_{\mathrm{LP}}\left(\Pi_{N} F_{0}, F_{0}\right) \rightarrow 0$ as $N \rightarrow \infty$.

- (B.1.3) (i) $\hat{Q}_{N}(F)$ is a measurable function of the data $\left\{y_{i}, x_{i}\right\}_{i=1}^{N}$ for all $F \in \mathcal{F}_{R(N)}$; (ii) $\hat{F}_{N}$ is well-defined and measurable.

- (B.1.4) (i) Let $\hat{c}^{Q}(R(N))=\sup _{F \in \mathcal{F}_{R(N)}}\left|\hat{Q}_{N}(F)-Q(F)\right|=o_{P}(1) ;($ ii)

$$
\frac{\max \left\{\hat{c}^{Q}(R(N)), \eta_{N},\left|Q\left(\Pi_{N} F_{0}\right)-Q\left(F_{0}\right)\right|\right\}}{\delta(N, R(N), \varepsilon)}=o(1) \text { for all } \varepsilon>0
$$

Then $d_{\mathrm{LP}}\left(\hat{F}_{N}, F_{0}\right) \longrightarrow o_{P}(1)$.

Proof. Under condition (B.1.3) (ii) $\hat{F}_{N}$ is well-defined and measurable. It follows that for any $\varepsilon>0$, 


$$
\begin{aligned}
& \operatorname{Pr}\left(d_{\mathrm{LP}}\left(\hat{F}_{N}, F_{0}\right) \geq \varepsilon\right) \\
\leq & \operatorname{Pr}\left(\inf _{F \in \mathcal{F}_{R(N)}: d_{\mathrm{LP}}\left(F, F_{0}\right) \geq \varepsilon} \hat{Q}_{N}(F) \leq \hat{Q}_{N}\left(\Pi_{N} F_{0}\right)+O\left(\eta_{N}\right)\right) \\
\leq & \operatorname{Pr}\left(\inf _{F \in \mathcal{F}_{R(N)}: d_{\mathrm{LP}}\left(F, F_{0}\right) \geq \varepsilon}\left\{Q(F)+\left(\hat{Q}_{N}(F)-Q(F)\right)\right\} \leq Q\left(\Pi_{N} F_{0}\right)+\left(\hat{Q}_{N}\left(\Pi_{N} F_{0}\right)-Q\left(\Pi_{N} F_{0}\right)\right)+O\left(\eta_{N}\right)\right) \\
\leq & \operatorname{Pr}\left(\inf _{F \in \mathcal{F}_{R(N)}: d_{\mathrm{LP}}\left(F, F_{0}\right) \geq \varepsilon}\left\{Q(F)-\left|\hat{Q}_{N}(F)-Q(F)\right|\right\} \leq Q\left(\Pi_{N} F_{0}\right)+\left|\hat{Q}_{N}\left(\Pi_{N} F_{0}\right)-Q\left(\Pi_{N} F_{0}\right)\right|+O\left(\eta_{N}\right)\right) \\
\leq & \operatorname{Pr}\left(\inf _{F \in \mathcal{F}_{R(N)}: d_{\mathrm{LP}}\left(F, F_{0}\right) \geq \varepsilon} Q(F) \leq 2 \hat{c}^{Q}(R(N))+Q\left(\Pi_{N} F_{0}\right)+O\left(\eta_{N}\right)\right) \\
\leq & \operatorname{Pr}\left(\inf _{F \in \mathcal{F}_{R(N)}: d_{\mathrm{LP}}\left(F, F_{0}\right) \geq \varepsilon} Q(F)-Q\left(F_{0}\right) \leq 2 \hat{c}^{Q}(R(N))+Q\left(\Pi_{N} F_{0}\right)-Q\left(F_{0}\right)+O\left(\eta_{N}\right)\right) \\
\leq & \operatorname{Pr}\left(\delta(N, R(N), \varepsilon) \leq 2 \hat{c}^{Q}(R(N))+\left|Q\left(\Pi_{N} F_{0}\right)-Q\left(F_{0}\right)\right|+O\left(\eta_{N}\right)\right)
\end{aligned}
$$

which goes to zero by condition (B.1.4).

In our problem we let

$$
\hat{Q}_{N}(F)=\frac{1}{N} \sum_{i=1}^{N}\left(y_{i}-\sum_{r=1}^{R} \theta^{r} g\left(x_{i}, \beta^{r}\right)\right)^{2}, F \in \mathcal{F}_{R(N)}
$$

and $Q(F)=E\left[\left(Y_{i}-\sum_{r=1}^{R} \theta^{r} g\left(X_{i}, \beta^{r}\right)\right)^{2}\right]$ for $F \in \mathcal{F}_{R(N)}$. Similarly we define

$$
\hat{Q}_{N}\left(F_{0}\right)=\frac{1}{N} \sum_{i=1}^{N}\left(y_{i}-\int g\left(x_{i}, \beta\right) d F_{0}(\beta)\right)^{2}
$$

and $Q\left(F_{0}\right)=E\left[\left(Y_{i}-\int g\left(X_{i}, \beta\right) d F_{0}(\beta)\right)^{2}\right]$. Note that since we do not use CP's optional penalization, we let $\bar{Q}_{N}(\cdot)=\bar{Q}(\cdot)=Q(\cdot)$ in CP's original Lemma B.1. Also note that by definition of our estimator as an extremum estimator, we have

$$
\hat{Q}_{N}\left(\hat{F}_{N}\right) \leq \inf _{F \in \mathcal{F}_{R(N)}} \hat{Q}_{N}(F)+O_{P}\left(\eta_{N}\right) \text { with } \eta_{N}=o(1)
$$


We start with the condition (B.1.1). The condition $Q\left(F_{0}\right)<\infty$ holds because $Q(F) \leq 1$ for all $F \in \mathcal{F}$. Next we will verify the condition

$$
\inf _{F \in \mathcal{F}_{R(N)}: d_{\mathrm{LP}}\left(F, F_{0}\right) \geq \varepsilon} Q(F)-Q\left(F_{0}\right) \geq \delta(N, R(N), \varepsilon)
$$

for each $N \geq 1, \varepsilon>0$, and some function $\delta(N, R(N), \varepsilon)$ to be defined where $\liminf _{N \rightarrow \infty} \delta(N, R(N), \varepsilon)>$ 0 (strictly positive). We will use our assumption of identification in Theorem 3.1. Let $m(x, F)=$ $P(x)-\int g(x, \beta) d F(\beta)$ and with abuse of notation, let $\int g(x, \beta) d F(\beta)=\sum_{r=1}^{R} \theta^{r} g\left(x, \beta^{r}\right)$ for $F \in \mathcal{F}_{R}$. First note that we have

$$
\begin{aligned}
Q(F) & =E\left[\left(Y-\int g(X, \beta) d F(\beta)\right)^{2}\right]=E\left[(Y-P(X)+m(X, F))^{2}\right] \\
& =E\left[(Y-P(X))^{2}\right]+2 E[(Y-P(X)) m(X, F)]+E\left[m(X, F)^{2}\right] \\
& =E\left[(Y-P(X))^{2}\right]+E\left[m(X, F)^{2}\right]
\end{aligned}
$$

where the last equality is obtained by applying the law of iterated expectation noting $E[Y-P(X) \mid X]=$ 0 . Therefore, for each $F \in \mathcal{F}_{R}$, we have

$$
Q(F)-Q\left(F_{0}\right)=E\left[m(X, F)^{2}\right]-E\left[m\left(X, F_{0}\right)^{2}\right]=E\left[m(X, F)^{2}\right]
$$

because $m\left(X, F_{0}\right)=0$ and the condition (23) holds due to our assumption of identification as the following argument shows. Consider $E\left[m(X, F)^{2}\right]$, with $m(X, F)$ defined above, as a map from $\mathcal{F}_{R(N)}$ to $\mathbb{R}^{+}$. As the true $F_{0}$ is not in the sieve space $\mathcal{F}_{R(N)}$, then $E\left[m(X, F)^{2}\right]$ takes on positive values for each $F \in \mathcal{F}_{R(N)}$, because the model is identified on a set $\tilde{\mathcal{X}}$ with positive probability. But $\mathcal{F}_{R(N)}$ is compact, so $E\left[m(X, F)^{2}\right]$ attains some minimum strictly positive value on $\mathcal{F}_{R(N)}$. Therefore, we can take $\delta(N, R(N), \varepsilon)>0$ for all $R(N) \geq 1$ with $\varepsilon>0$.

Next we consider (B.1.2). First note that $\left(\mathbf{F}, d_{\mathrm{LP}}\right)$ is a metric space and we have $\mathcal{F}_{R} \subseteq$ $\mathcal{F}_{R+1} \subseteq \mathcal{F}$ for all $R \geq 1$ by construction of our sieve space. Then we claim that there exist a sequence of functions $\Pi_{N} F_{0} \in \mathcal{F}_{R(N)}$ such that $d_{\mathrm{LP}}\left(\Pi_{N} F_{0}, F_{0}\right) \rightarrow 0$ as $N \rightarrow \infty$ as follows. First, $\mathcal{B}^{R(N)}$ becomes dense in $\mathcal{B}$ by assumption of the theorem. Second, $\mathcal{F}_{R(N)}$ becomes dense in $\mathcal{F}$ because the set of distributions on a dense subset $\mathcal{B}^{R(N)} \subset \mathcal{B}$ is itself dense. To see this, remember that the class of all distributions with finite support is dense in the class of all distributions (Aliprantis and Border 2006, Theorem 15.10). Any distribution with finite support 
can be approximated using a finite support in a dense subset $\mathcal{B}^{R(N)}$ (Huber 2004).

Next, to show (B.1.3) holds, we resort to Remark B.1. (1) (a) of CP. First note that $\mathcal{F}_{R}$ is a compact subset of $\mathbf{F}$ for each $R$. This is because the simplex

$$
\Delta_{R(N)}=\left\{\theta=\left(\theta^{1}, \ldots, \theta^{R(N)}\right) \mid \theta^{r} \geq 0, \sum_{r=1}^{R(N)} \theta^{r}=1\right\}
$$

itself is compact in the definition of $\mathcal{F}_{R}$. Second we need to show that for any data $\left\{y_{i}, x_{i}\right\}_{i=1}^{N}$, $\hat{Q}_{N}(F)$ is lower semicontinuous on $\mathcal{F}_{R}$ for each $R \geq 1$. Again with abuse of notation, let $\int g(x, \beta) d F=\sum_{r=1}^{R} \theta^{r} g\left(x, \beta^{r}\right)$ for $F \in \mathcal{F}_{R}$. Then, for any $F_{1}, F_{2} \in \mathcal{F}_{R(N)}$, applying the triangle inequality gives us

$$
\begin{gathered}
\left|\hat{Q}_{N}\left(F_{1}\right)-\hat{Q}_{N}\left(F_{2}\right)\right| \\
\leq 2 \frac{1}{N} \sum_{i=1}^{N} y_{i} \int g\left(x_{i}, \beta\right) d\left|F_{1}-F_{2}\right|+\frac{1}{N} \sum_{i=1}^{N}\left(\int g\left(x_{i}, \beta\right) d\left(F_{1}+F_{2}\right)\right)\left(\int g\left(x_{i}, \beta\right) d\left|F_{1}-F_{2}\right|\right) .
\end{gathered}
$$

Because $y_{i}, g\left(x_{i}, \beta\right)$, and $\int g\left(x_{i}, \beta\right) d F(\beta)$ are uniformly bounded, the above implies $\left|\hat{Q}_{N}\left(F_{1}\right)-\hat{Q}_{N}\left(F_{2}\right)\right| \rightarrow$ 0 as $F_{1} \rightarrow F_{2}$. This means that $\hat{Q}_{N}(F)$ is continuous on $\mathcal{F}_{R}$; continuity is stronger than lower semicontinuity.

Next there are two conditions to verify in (B.1.4). We first focus on the uniform convergence of $\hat{Q}_{N}(F)$ to $Q(F)$ for $F \in \mathcal{F}_{R(N)}$,

$$
\sup _{F \in \mathcal{F}_{R(N)}}\left|\hat{Q}_{N}(F)-Q(F)\right|=o_{P}(1) .
$$

It is convenient to view $\hat{Q}_{N}(F)$ and $Q(F)$ as functions of $\theta \in \Delta_{R(N)}$ and so to write $\hat{Q}_{N}(\theta)$ and $Q(\theta)$, respectively. Then we can show that the class of measurable functions

$$
\mathcal{G}=\left\{l(y, x, \theta)=\left(y-\sum_{r=1}^{R} \theta^{r} g\left(x, \beta^{r}\right)\right)^{2}: \theta \in \Delta_{R(N)}\right\}
$$

is P-Donsker due to Theorems 2.5.6 and 2.7.11 of van der Vaart and Weller (1996), noting that 
(i) $l(y, x, \theta)$ is uniformly bounded by 1 and is Lipschitz in $\theta$, i.e.,

$$
\begin{aligned}
\left|l\left(y, x, \theta_{1}\right)-l\left(y, x, \theta_{2}\right)\right| \leq & 2 y \sum_{r=1}^{R} g\left(x, \beta^{r}\right)\left|\theta_{1}^{r}-\theta_{2}^{r}\right| \\
& +\sum_{r=1}^{R}\left(g\left(x, \beta^{r}\right)\left(\theta_{1}^{r}+\theta_{2}^{r}\right)\right) \sum_{r=1}^{R}\left(g\left(x, \beta^{r}\right)\left|\theta_{1}^{r}-\theta_{2}^{r}\right|\right) \\
\leq & M(\cdot) \sum_{r=1}^{R}\left|\theta_{1}^{r}-\theta_{2}^{r}\right| \leq M(\cdot) \sqrt{R}\left\|\theta_{1}-\theta_{2}\right\|_{E}
\end{aligned}
$$

with some function $E\left[M(\cdot)^{2}\right]<\infty$ and $\|\cdot\|_{E}$ denotes the Euclidean norm ${ }^{22}$ and (ii) $\Delta_{R(N)}$ is a compact subset of $\mathbb{R}^{R(N)}$.

As measures of complexity of spaces, let $\mathbf{N}(\varepsilon, \mathcal{T},\|\cdot\|)$ denote the covering number of the set $\mathcal{T}$ with balls of radius $\varepsilon$ with a norm $\|\cdot\|$ and let $\mathbf{N}_{[]}(\varepsilon, \mathcal{T},\|\cdot\|)$ denote the bracketing number of the set $\mathcal{T}$ with $\varepsilon$-brackets.

We take $M(\cdot)=4$ (noting $y, g(\cdot)$, and $\sum_{r=1}^{R} g\left(x, \beta^{r}\right) \theta^{r}$ are uniformly bounded by 1 ). Then due to Theorem 2.7.11 of van der Vaart and Wellner $(1996)$, we have $\mathbf{N}_{[]}(\varepsilon, \mathcal{G},\|\cdot\|) \leq$ $\mathbf{N}\left(\frac{\varepsilon}{8 \sqrt{R}}, \Delta_{R},\|\cdot\|_{E}\right)=\left(\frac{8 \sqrt{R}}{\varepsilon}\right)^{R}$ for any norm $\|\cdot\|$. It follows that

$$
\int_{0}^{1} \sqrt{\log \mathbf{N}_{[]}(\varepsilon, \mathcal{G},\|\cdot\|)} d \varepsilon=O(R \log R)
$$

therefore we find $\hat{c}^{Q}(R(N))=\frac{R(N) \log R(N)}{\sqrt{N}}$ and need $\frac{R(N) \log R(N)}{\sqrt{N}} \rightarrow 0$ to satisfy the uniform convergence condition (25).

To satisfy the second condition in (B.1.4), it suffices to show all three terms in $\max \{\cdot\}$ go to zero, provided that $\liminf _{N \rightarrow \infty} \delta(N, R(N), \varepsilon)>0$. We have shown the uniform convergence of the sample criterion function and we can take $\eta_{N}$ small enough. We also have

$$
\left|Q\left(\Pi_{N} F_{0}\right)-Q\left(F_{0}\right)\right|=o(1)
$$

which is trivially satisfied from the continuity of $Q(F)$ and $\Pi_{N} F_{0} \rightarrow F_{0}$ from (B.1.2) (ii).

Next we formally prove $\lim \inf _{N \rightarrow \infty} \delta(N, R(N), \varepsilon)>0$. Note that there exists an $F_{R(N)}^{*} \in$

\footnotetext{
${ }^{22}$ In the above, the first inequality is obtained by the triangle inequality and the second inequality holds due to the Cauchy-Schwarz inequality.
} 
$\mathcal{F}_{R(N)}$ such that $\left.d_{\mathrm{LP}}\left(F_{R(N)}^{*}, F_{0}\right)\right) \geq \varepsilon$ and

$$
F_{R(N)}^{*}=\arg \min _{F \in \mathcal{F}_{R(N)}: d_{\mathrm{LP}}\left(F, F_{0}\right) \geq \varepsilon} Q(F)-Q\left(F_{0}\right)
$$

since $\mathcal{F}_{R(N)}$ is compact. Then from (24), we obtain

$$
\begin{aligned}
Q\left(F_{R(N)}^{*}\right)-Q\left(F_{0}\right)= & E\left[m\left(X, F_{R(N)}^{*}\right)^{2}\right]=E\left[\left(m\left(X, F_{R(N)}^{*}\right)-m\left(X, F_{0}\right)\right)^{2}\right] \\
= & E\left[\left(m\left(X, F_{R(N)}^{*}\right)-m\left(X, \Pi_{N} F_{0}\right)\right)^{2}\right]+E\left[\left(m\left(X, \Pi_{N} F_{0}\right)-m\left(X, F_{0}\right)\right)^{2}\right] \\
& +2 E\left[\left(m\left(X, F_{R(N)}^{*}\right)-m\left(X, \Pi_{N} F_{0}\right)\right)\left(m\left(X, \Pi_{N} F_{0}\right)-m\left(X, F_{0}\right)\right)\right] \\
= & E\left[\left(m\left(X, F_{R(N)}^{*}\right)-m\left(X, \Pi_{N} F_{0}\right)\right)^{2}\right]+o(1), \\
= & E\left[\left(\sum_{r=1}^{R(N)} g\left(X, \beta^{r}\right)\left(\theta_{*}^{r}-\Pi_{N} \theta_{0}^{r}\right)\right)^{2}\right]+o(1) \\
\geq & \xi_{\min }(R(N)) \sum_{r=1}^{R(N)}\left(\theta_{*}^{r}-\Pi_{N} \theta_{0}^{r}\right)^{2}+o(1)
\end{aligned}
$$

where $F_{R(N)}^{*}(\beta)=\sum_{r=1}^{R} \theta_{*}^{r} 1\left[\beta^{r} \leq \beta\right], \Pi_{N} F_{0}(\beta)=\sum_{r=1}^{R} \Pi_{N} \theta_{0}^{r} 1\left[\beta^{r} \leq \beta\right]$, and $\xi_{\min }(R(N))$ denotes the smallest eigenvalue of the matrix $\left(E\left[g\left(X, \beta^{r}\right) g\left(X, \beta^{r^{\prime}}\right)\right]\right)_{1 \leq r, r^{\prime} \leq R(N)}$. In the above, we obtain the fourth equality because $\Pi_{N} F_{0} \rightarrow F_{0}$ and $m(X, F)$ is continuous in $F$, applying the dominated convergence theorem.

We will prove $\lim \inf _{N \rightarrow \infty} \delta(N, R(N), \varepsilon)>0$ by contradiction. Now suppose that $Q\left(F_{R(N)}^{*}\right)-$ $Q\left(F_{0}\right) \rightarrow 0$. Then we must have $\left|\theta_{*}^{r}-\Pi_{N} \theta_{0}^{r}\right| \rightarrow 0$ for all $r=1, \ldots, R(N)$ because we assume the matrix $\left(E\left[g\left(X, \beta^{r}\right) g\left(X, \beta^{r^{\prime}}\right)\right]\right)_{1 \leq r, r^{\prime} \leq R(N)}$ is positive definite and its smallest eigenvalue is bounded away from zero uniformly in $R(N)$. It follows that $d_{\mathrm{LP}}\left(F_{R(N)}^{*}, \Pi_{N} F_{0}\right) \rightarrow 0$, which contradicts the fact that $d_{\mathrm{LP}}\left(F_{R(N)}^{*}, F_{0}\right) \geq \varepsilon>0$ because $d_{\mathrm{LP}}\left(F_{R(N)}^{*}, F_{0}\right) \leq d_{\mathrm{LP}}\left(F_{R(N)}^{*}, \Pi_{N} F_{0}\right)+$ $d_{\mathrm{LP}}\left(\Pi_{N} F_{0}, F_{0}\right)$. Therefore, we conclude that $\liminf _{N \rightarrow \infty} \delta(N, R(N), \varepsilon)$ is strictly positive.

We have verified all the conditions in Lemma B.1 of CP and this completes the consistency proof. 


\section{B Proof of the Asymptotic Bounds}

We let $R=R(N)$. We also let $C, C_{1}, C_{2}, \ldots$ denote generic positive constants. We let $\xi_{\min }(j, R)$ denote the smallest eigenvalue of $\left(E\left[g_{j}\left(X_{i}, \beta^{r}\right) g_{j}\left(X_{i}, \beta^{r^{\prime}}\right)\right]\right)_{1 \leq r, r^{\prime} \leq R}$. We use $\operatorname{diag}(A)$ to denote a diagonal matrix composed of diagonal elements of a matrix $A$. We often use the following inequality (denoted by RCS for the Cauchy-Schwarz inequality for $R$ terms in a sum): $\sum_{r=1}^{R} W_{r} \leq \sqrt{R} \sqrt{\sum_{r=1}^{R} W_{r}^{2}}$ for a sequence $W_{r}$ 's.

We first prove preliminary lemmas that are useful to prove Theorem 4.1 and Theorem 4.2. We define

$\Psi_{N, R}=\left(\frac{1}{N} \sum_{i=1}^{N} g_{j}\left(x_{i}, \beta^{r}\right) g_{j}\left(x_{i}, \beta^{r^{\prime}}\right)\right)_{1 \leq r, r^{\prime} \leq R}$ and $\Psi_{R}=\left(E\left[g_{j}\left(X_{i}, \beta^{r}\right) g_{j}\left(X_{i}, \beta^{r^{\prime}}\right)\right]\right)_{1 \leq r, r^{\prime} \leq R}$, where we suppress $\Psi_{N, R}$ and $\Psi_{R}$ 's dependence on $j$ for notational simplicity.

Lemma B.1 Suppose Assumption 4.1 and 4.2 hold. Then

$$
\begin{array}{r}
\min \left[\operatorname{Pr}\left\{\left\|g_{j}\left(\cdot, \beta^{r}\right)\right\|_{L_{2, N}}^{2} \leq 2\left\|g_{j}\left(\cdot, \beta^{r}\right)\right\|_{L_{2}}^{2}, \forall r\right\}, \operatorname{Pr}\left\{\left\|g_{j}\left(\cdot, \beta^{r}\right)\right\|_{L_{2}} \leq 2\left\|g_{j}\left(\cdot, \beta^{r}\right)\right\|_{L_{2, N}}, \forall r\right\}\right] \geq \\
1-R \exp \left(-C_{1} \frac{N c_{0}^{4}}{\zeta_{0}^{4}}\right) .
\end{array}
$$

Proof. The claim follows from the union bound applied to the $r$-specific events and Hoeffding's inequality.

Lemma B.1 suggests that as $N$ increases, $\left\|g_{j}\left(\cdot, \beta^{r}\right)\right\|_{L_{2, N}}$ and $\left\|g_{j}\left(\cdot, \beta^{r}\right)\right\|_{L_{2}}$ get closer to each other with probability exponentially approaching to one. Lemma B.1 implies that $\operatorname{diag}\left(\Psi_{N, R}\right) \leq$ $2 \operatorname{diag}\left(\Psi_{R}\right)$ holds with probability approaching to one because the $\left\|g_{j}\left(\cdot, \beta^{r}\right)\right\|_{L_{2, N}}^{2}$ 's are diagonal elements of $\Psi_{N, R}$ and the $\left\|g_{j}\left(\cdot, \beta^{r}\right)\right\|_{L_{2}}^{2}$ 's are diagonal elements of $\Psi_{R}$.

The purpose of the following Lemma B.2 is to show that $\Psi_{N, R} \geq \Psi_{R} / 2$ holds with probability approaching to one. This in turn shows that $\Psi_{N, R}$ is nonsingular with probability approaching to one.

Lemma B.2 Let $\mathcal{G}=\operatorname{span}\left\{g_{j}\left(\cdot, \beta^{1}\right), \ldots, g_{j}\left(\cdot, \beta^{R}\right)\right\}$ be the linear space spanned by some func- 
tions $g_{j}\left(\cdot, \beta^{1}\right), \ldots, g_{j}\left(\cdot, \beta^{R}\right)$. Note that obviously $\mathcal{H}_{R}^{j} \subset \mathcal{G}$ where $\mathcal{H}_{R}^{j}$, is defined in (16). Then

$$
\operatorname{Pr}\left\{\sup _{\mu \in \mathcal{G} \backslash\{0\}} \frac{\|\mu\|_{L_{2}}^{2}}{\|\mu\|_{L_{2, N}}^{2}}>2\right\} \leq R^{2} \exp \left(-C_{2} \frac{N}{\zeta_{0}^{4} R^{2}}\right)
$$

for some constant $C$.

Proof. Let $\phi_{1}, \ldots, \phi_{M}$ be an orthonormal basis of $\mathcal{G}$ in $L_{2}$ with $M \leq R$. Also let $\bar{\rho}(D)$ denote the following quantity for a symmetric matrix $D$ :

$$
\bar{\rho}(D)=\sup \sum_{l}\left|a_{l}\right| \sum_{l^{\prime}}\left|a_{l^{\prime}}\right|\left|D_{l, l^{\prime}}\right|
$$

where the sup is taken over sequences $\left\{a_{l}\right\}_{l=1}^{M}$ with $\sum_{l=1}^{M} a_{l}^{2}=1$. Then, following Lemma 5.2 in Baraud (2002), we have

$$
\operatorname{Pr}\left\{\sup _{\mu \in \mathcal{G} \backslash\{0\}} \frac{\|\mu\|_{L_{2}}^{2}}{\|\mu\|_{L_{2, N}}^{2}}>c\right\} \leq M^{2} \exp \left(-N \frac{\left(\varpi_{0}-c^{-1}\right)^{2}}{4 \varpi_{1} \max \left\{\bar{\rho}^{2}(A), \bar{\rho}(B)\right\}}\right)
$$

where $A_{l, l^{\prime}}=\sqrt{E\left[\phi_{l}^{2} \phi_{l^{\prime}}^{2}\right]}$ and $B_{l, l^{\prime}}=\left\|\phi_{l} \phi_{l^{\prime}}\right\|_{\infty}$ for $l, l^{\prime}=1, \ldots, M$ and $\varpi_{0}$ and $\varpi_{1}$ denote the lower bound and upper bound of the density of $X$, respectively. We find $\left|A_{l, l^{\prime}}\right| \leq \zeta_{0}^{2}$ and $\left|B_{l, l^{\prime}}\right| \leq \zeta_{0}^{2}$. It follows that

$$
\bar{\rho}(A) \leq \zeta_{0}^{2} \sup \sum_{l}\left|a_{l}\right| \sum_{l^{\prime}}\left|a_{l^{\prime}}\right|=\zeta_{0}^{2} \sup \left(\sum_{l}\left|a_{l}\right|\right)^{2} \leq \zeta_{0}^{2} \sup M \sum_{l}\left|a_{l}\right|^{2}=\zeta_{0}^{2} M \leq \zeta_{0}^{2} R
$$

where $\left(\sum_{l}\left|a_{l}\right|\right)^{2} \leq M \sum_{l}\left|a_{l}\right|^{2}$ holds by the Cauchy-Schwarz inequality (and note $\sum_{l}\left|a_{l}\right|^{2}=1$ in our construction). Similarly we have $\bar{\rho}(B) \leq \zeta_{0}^{2} R$. Noting $M \leq R$, from (26), we conclude

$$
\operatorname{Pr}\left\{\sup _{\mu \in \mathcal{G} \backslash\{0\}} \frac{\|\mu\|_{L_{2}}^{2}}{\|\mu\|_{L_{2, N}}^{2}}>2\right\} \leq R^{2} \exp \left(-C_{2} \frac{N}{\zeta_{0}^{4} R^{2}}\right)
$$

Next combining Lemma B.1 and Lemma B.2, we obtain a bound for the probability that the matrix $\Psi_{N, R}$ is not smaller than a diagonal matrix in the semi-positive definite sense. 
Lemma B.3 Suppose Assumption 4.1 and 4.2 hold. Then,

$$
\operatorname{Pr}\left\{\Psi_{N, R}-\frac{\xi_{\min }(R)}{4 \zeta_{0}^{2}} \operatorname{diag}\left(\Psi_{N, R}\right) \geq 0\right\} \geq 1-R \exp \left(-C_{1} \frac{N c_{0}^{4}}{\zeta_{0}^{4}}\right)-R^{2} \exp \left(-C_{2} \frac{N}{\zeta_{0}^{4} R^{2}}\right)
$$

Proof. First, note that $\Psi_{R}-\frac{\xi_{\min }(R)}{\zeta_{0}^{2}} \operatorname{diag}\left(\Psi_{R}\right) \geq 0$ (positive semi-definite) because $\Psi_{R}$ is a positive definite matrix by Assumption 4.2 (ii) and a well-known result involving eigenvalues. Now let $\mathcal{G}$ be the linear space spanned by $g_{j}\left(\cdot, \beta^{1}\right), \ldots, g_{j}\left(\cdot, \beta^{R}\right)$. Now note that under the event $\left\|g_{j}\left(\cdot, \beta^{r}\right)\right\|_{L_{2, N}}^{2} \leq 2\left\|g_{j}\left(\cdot, \beta^{r}\right)\right\|_{L_{2}}^{2} \forall r=1, \ldots, R$, we have $\operatorname{diag}\left(\Psi_{N, R}\right) \leq 2 \operatorname{diag}\left(\Psi_{R}\right)$ and note that under the event $\left\{\sup _{\mu \in \mathcal{G} \backslash\{0\}} \frac{\|\mu\|_{L_{2}}^{2}}{\|\mu\|_{L_{2, N}}^{2}}>2\right\}$, we have $\Psi_{N, R} \geq \Psi_{R} / 2$. Therefore, under the event

$$
\begin{gathered}
\left\{\left\|g_{j}\left(\cdot, \beta^{r}\right)\right\|_{L_{2, N}}^{2} \leq 2\left\|g_{j}\left(\cdot, \beta^{r}\right)\right\|_{L_{2}}^{2}\right\}, \forall r=1, \ldots, R \cap\left\{\sup _{\mu \in \mathcal{G} \backslash\{0\}} \frac{\|\mu\|_{L_{2}}^{2}}{\|\mu\|_{L_{2, N}}^{2}}>2\right\}, \text { we have } \\
\Psi_{N, R}-\frac{\xi_{\min }(R)}{4 \zeta_{0}^{2}} \operatorname{diag}\left(\Psi_{N, R}\right) \geq \Psi_{R} / 2-\frac{\xi_{\min }(R)}{4 \zeta_{0}^{2}} 2 \operatorname{diag}\left(\Psi_{R}\right) \geq 0
\end{gathered}
$$

since $\Psi_{R}-\frac{\xi_{\min }(R)}{\zeta_{0}^{2}} \operatorname{diag}\left(\Psi_{R}\right) \geq 0$. It follows that

$$
\begin{aligned}
& 1-\operatorname{Pr}\left\{\Psi_{N, R}-\frac{\xi_{\min }(R)}{4 \zeta_{0}^{2}} \operatorname{diag}\left(\Psi_{N, R}\right) \geq 0\right\} \leq \\
& 1-\operatorname{Pr}\left\{\left\|g_{j}\left(\cdot, \beta^{r}\right)\right\|_{L_{2, N}}^{2} \leq 2\left\|g_{j}\left(\cdot, \beta^{r}\right)\right\|_{L_{2}}^{2}, \forall r=1, \ldots, R\right\}+1-\operatorname{Pr}\left\{\sup _{\mu \in \mathcal{G} \backslash\{0\}} \frac{\|\mu\|_{L_{2}}^{2}}{\|\mu\|_{L_{2, N}}^{2}}>2\right\} \\
& \leq R \cdot \exp \left(-C_{1} \frac{N c_{0}^{4}}{\zeta_{0}^{4}}\right)+R^{2} \cdot \exp \left(-C_{2} \frac{N}{\zeta_{0}^{4} R^{2}}\right)
\end{aligned}
$$

where the last inequality is obtained from Lemma B.1 and Lemma B.2.

Lemma B.4 Suppose Assumptions 4.1 and 4.2 hold. Then, for given positive sequence $\eta_{N}$

$$
\begin{aligned}
& \operatorname{Pr}\left\{\left|\frac{1}{N} \sum_{i=1}^{N} e_{j, i} g_{j}\left(x_{i}, \beta^{r}\right)\right| \leq \eta_{N}\left\|g_{j}\left(\cdot, \beta^{r}\right)\right\|_{L_{2, N}} \text { for all } r=1, \ldots, R\right\} \\
\geq & 1-R \cdot \exp \left(-\frac{N \eta_{N}^{2} c_{0}^{2}}{8 \zeta_{0}^{2}}\right)-R \cdot \exp \left(-C_{1} \frac{N c_{0}^{4}}{\zeta_{0}^{4}}\right) .
\end{aligned}
$$


Proof. Hoeffding (1963)'s inequality implies that

$$
\begin{aligned}
& E_{X}\left[\operatorname{Pr}\left\{\left|\frac{1}{N} \sum_{i} e_{j, i} g_{j}\left(X_{i}, \beta^{r}\right)\right| \geq \eta_{N}\left\|g_{j}\left(\cdot, \beta^{r}\right)\right\|_{L_{2, N}}, \forall r \leq R \mid X_{1}, \ldots, X_{N}\right\}\right] \\
\leq & E_{X}\left[\sum_{r=1}^{R} \exp \left(-2 N \eta_{N}^{2}\left\|g_{j}\left(\cdot, \beta^{r}\right)\right\|_{L_{2, N}}^{2} / 4 \zeta_{0}^{2}\right)\right]
\end{aligned}
$$

because $E\left[e_{j, i} g_{j}\left(X_{i}, \beta^{r}\right) \mid X_{1}, \ldots, X_{N}\right]=0$ and because choice probabilities lie in $[0,1],-\zeta_{0} \leq$ $e_{j, i} g_{j}\left(X_{i}, \beta^{r}\right) \leq \zeta_{0}$ uniformly.

Now note under the event $\left\{\left\|g_{j}\left(\cdot, \beta^{r}\right)\right\|_{L_{2}} \leq 2\left\|g_{j}\left(\cdot, \beta^{r}\right)\right\|_{L_{2, N}}, \forall r=1, \ldots, R\right\}$,

$$
\begin{aligned}
\sum_{r=1}^{R} \exp \left(-N \eta_{N}^{2}\left\|g_{j}\left(\cdot, \beta^{r}\right)\right\|_{L_{2, N}}^{2} / 2 \zeta_{0}^{2}\right) & \leq \sum_{r=1}^{R} \exp \left(-N \eta_{N}^{2}\left\|g_{j}\left(\cdot, \beta^{r}\right)\right\|_{L_{2}}^{2} / 8 \zeta_{0}^{2}\right) \\
& \leq \sum_{r=1}^{R} \exp \left(-N \eta_{N}^{2} c_{0}^{2} / 8 \zeta_{0}^{2}\right)=R \exp \left(-N \eta_{N}^{2} c_{0}^{2} / 8 \zeta_{0}^{2}\right)
\end{aligned}
$$

From (27)-(28), combining the bound for $\operatorname{Pr}\left\{\left\|g_{j}\left(\cdot, \beta^{r}\right)\right\|_{L_{2}}>2\left\|g_{j}\left(\cdot, \beta^{r}\right)\right\|_{L_{2, N}}, \forall r=1, \ldots, R\right\}=$ $R \exp \left(-C_{1} \frac{N c_{0}^{4}}{\zeta_{0}^{4}}\right)$ in Lemma B.1, the claim follows.

The following Lemma B.5 decomposes the approximation error into a bias term and a variance term.

Lemma B.5 Suppose Assumptions 4.1 and 4.2 hold. Then, for any $N \geq 1, R \geq 2$, and $a>1$, we have for all $\theta \in \Delta_{N}$,

$\frac{1}{J} \sum_{j=1}^{J}\left\|\mu_{\widehat{\theta}}\left(j, x_{i}\right)-P\left(j \mid x_{i}, f_{0}\right)\right\|_{L_{2, N}}^{2} \leq \frac{a+1}{a-1} \frac{1}{J} \sum_{j=1}^{J}\left\|\mu_{\theta}\left(j, x_{i}\right)-P\left(j \mid x_{i}, f_{0}\right)\right\|_{L_{2, N}}^{2}+\frac{\zeta_{0}^{2}}{\xi_{\min }(R)} \frac{8 a^{2}}{a-1} \eta_{N}^{2} R$,

where the inequality holds with probability greater than $1-p_{N, R}$,

$$
p_{N, R}=R J \exp \left(-\frac{N \eta_{N}^{2} c_{0}^{2}}{8 \zeta_{0}^{2}}\right)+2 R J \exp \left(-C_{1} \frac{N c_{0}^{4}}{\zeta_{0}^{4}}\right)+R^{2} J \exp \left(-C_{2} \frac{N}{\zeta_{0}^{2} R^{2}}\right)
$$


Proof. Because $\mu_{\widehat{\theta}}(\cdot)$ (i.e., $\left.\widehat{\theta}\right)$ is the solution of the minimization problem in (15), we have

$$
\sum_{j=1}^{J}\left\|\mu_{\widehat{\theta}}\left(j, x_{i}\right)-y_{i, j}\right\|_{L_{2, N}}^{2} \leq \sum_{j=1}^{J}\left\|\mu_{\theta}\left(j, x_{i}\right)-y_{i, j}\right\|_{L_{2, N}}^{2}
$$

for any $\theta \in \Delta_{R(N)}$. Now note that

$$
\begin{aligned}
& \sum_{j=1}^{J}\left\|\mu_{\widehat{\theta}}\left(j, x_{i}\right)-y_{i, j}\right\|_{L_{2, N}}^{2}=\sum_{j=1}^{J}\left\|\mu_{\widehat{\theta}}\left(j, x_{i}\right)-P\left(j \mid x_{i}, f_{0}\right)+P\left(j \mid x_{i}, f_{0}\right)-y_{i, j}\right\|_{L_{2, N}}^{2} \\
= & \sum_{j=1}^{J}\left\|\mu_{\widehat{\theta}}\left(j, x_{i}\right)-P\left(j \mid x_{i}, f_{0}\right)\right\|_{L_{2, N}}^{2}-\sum_{j=1}^{J} \frac{2}{N} \sum_{i} e_{j, i}\left(\mu_{\widehat{\theta}}\left(j, x_{i}\right)-P\left(j \mid x_{i}, f_{0}\right)\right)+\sum_{j=1}^{J}\left\|e_{j, i}\right\|_{L_{2, N}}^{2},
\end{aligned}
$$

where we use the definition $e_{j, i}=y_{i, j}-P\left(j \mid x_{i}, f_{0}\right)$. Similarly we obtain

$$
\begin{aligned}
& \sum_{j=1}^{J}\left\|\mu_{\theta}\left(j, x_{i}\right)-y_{i, j}\right\|_{L_{2, N}}^{2}= \\
& \sum_{j=1}^{J}\left\|\mu_{\theta}\left(j, x_{i}\right)-P\left(j \mid x_{i}, f_{0}\right)\right\|_{L_{2, N}}^{2}-\sum_{j=1}^{J} \frac{2}{N} \sum_{i} e_{j, i}\left(\mu_{\theta}\left(j, x_{i}\right)-P\left(j \mid x_{i}, f_{0}\right)\right)+\sum_{j=1}^{J}\left\|e_{j, i}\right\|_{L_{2, N}}^{2} .
\end{aligned}
$$

Subtracting (31) from (30) and noting (31) $\geq(30)$ from (29), we obtain

$$
\begin{aligned}
& \sum_{j=1}^{J}\left\|\mu_{\widehat{\theta}}\left(j, x_{i}\right)-P\left(j \mid x_{i}, f_{0}\right)\right\|_{L_{2, N}}^{2} \\
\leq & \sum_{j=1}^{J}\left\|\mu_{\theta}\left(j, x_{i}\right)-P\left(j \mid x_{i}, f_{0}\right)\right\|_{L_{2, N}}^{2}+\sum_{j=1}^{J} \frac{2}{N} \sum_{i} e_{j, i}\left(\mu_{\widehat{\theta}}\left(j, x_{i}\right)-\mu_{\theta}\left(j, x_{i}\right)\right) .
\end{aligned}
$$

Now let $V_{N, r}(j)=\frac{1}{N} \sum_{i=1}^{N} e_{j, i} g_{j}\left(x_{i}, \beta^{r}\right)$. Then, we have

$$
\frac{1}{N} \sum_{i} e_{j, i}\left(\mu_{\widehat{\theta}}\left(j, x_{i}\right)-\mu_{\theta}\left(j, x_{i}\right)\right)=\sum_{r=1}^{R} V_{N, r}(j)\left(\widehat{\theta}^{r}-\theta^{r}\right)
$$


by construction of $\mu_{\widehat{\theta}}$ and $\mu_{\theta}$ and we obtain by the triangle inequality,

$\sum_{j=1}^{J}\left\|\mu_{\widehat{\theta}}\left(j, x_{i}\right)-P\left(j \mid x_{i}, f_{0}\right)\right\|_{L_{2, N}}^{2} \leq \sum_{j=1}^{J}\left\|\mu_{\theta}\left(j, x_{i}\right)-P\left(j \mid x_{i}, f_{0}\right)\right\|_{L_{2, N}}^{2}+2 \sum_{j=1}^{J} \sum_{r=1}^{R}\left|V_{N, r}(j)\right|\left|\widehat{\theta}^{r}-\theta^{r}\right|$.

Define two events $E_{0, j}=\bigcap_{r=1}^{R}\left\{\left|V_{N, r}(j)\right| \leq \eta_{N}\left\|g_{j}\left(\cdot, \beta^{r}\right)\right\|_{L_{2, N}}\right\}$ for some positive sequence $\eta_{N}$ and $E_{1, j}=\left\{\Psi_{N, R}-\frac{\xi_{\min }(R)}{4 \zeta_{0}^{2}} \operatorname{diag}\left(\Psi_{N, R}\right) \geq 0\right\}$. Then, under $E_{0, j} \cap E_{1, j}$, we note

$$
\begin{aligned}
\sum_{r=1}^{R} V_{N, r}^{2}(j)\left(\widehat{\theta}^{r}-\theta^{r}\right)^{2} & \leq \eta_{N}^{2} \sum_{r=1}^{R}\left\|g_{j}\left(\cdot, \beta^{r}\right)\right\|_{L_{2, N}}^{2}\left(\widehat{\theta}^{r}-\theta^{r}\right)^{2} \\
& =\eta_{N}^{2} \frac{1}{N} \sum_{i=1}^{N} \sum_{r=1}^{R}\left(\widehat{\theta}^{r}-\theta^{r}\right)^{2} g_{j}\left(x_{i}, \beta^{r}\right)^{2} \\
& =\eta_{N}^{2}(\widehat{\theta}-\theta)^{\prime} \operatorname{diag}\left(\Psi_{N, R}(j)\right)(\widehat{\theta}-\theta) \\
& \leq \eta_{N}^{2}\left(\xi_{\min }(R) / 4 \zeta_{0}^{2}\right)^{-1}(\widehat{\theta}-\theta)^{\prime} \Psi_{N, R}(j)(\widehat{\theta}-\theta) \\
& =\eta_{N}^{2}\left(\xi_{\min }(R) / 4 \zeta_{0}^{2}\right)^{-1}\left\|\mu_{\widehat{\theta}}\left(j, x_{i}\right)-\mu_{\theta}\left(j, x_{i}\right)\right\|_{L_{2, N}}^{2} .
\end{aligned}
$$

The above uses Lemma B.3. From (32) and (33), it follows that on $\cap_{j=1}^{J}\left(E_{0, j} \cap E_{1, j}\right)$

$$
\begin{aligned}
& \sum_{j=1}^{J}\left\|\mu_{\widehat{\theta}}\left(j, x_{i}\right)-P\left(j \mid x_{i}, f_{0}\right)\right\|_{L_{2, N}}^{2} \\
\leq & \sum_{j=1}^{J}\left\|\mu_{\theta}\left(j, x_{i}\right)-P\left(j \mid x_{i}, f_{0}\right)\right\|_{L_{2, N}}^{2}+2 \sum_{j=1}^{J} \sum_{r=1}^{R}\left|V_{N, r}(j)\right|\left|\widehat{\theta}^{r}-\theta^{r}\right| \\
\leq & \sum_{j=1}^{J}\left\|\mu_{\theta}\left(j, x_{i}\right)-P\left(j \mid x_{i}, f_{0}\right)\right\|_{L_{2, N}}^{2}+2 \sum_{j=1}^{J} \sqrt{R} \sqrt{\sum_{r=1}^{R} V_{N, r}^{2}(j)\left(\widehat{\theta}^{r}-\theta^{r}\right)^{2}} \\
\leq & \sum_{j=1}^{J}\left\|\mu_{\theta}\left(j, x_{i}\right)-P\left(j \mid x_{i}, f_{0}\right)\right\|_{L_{2, N}}^{2}+2 \sum_{j=1}^{J} \eta_{N} \sqrt{R\left(\frac{\xi_{\min }(R)}{4 \zeta_{0}^{2}}\right)^{-1}}\left\|\mu_{\widehat{\theta}}\left(j, x_{i}\right)-\mu_{\theta}\left(j, x_{i}\right)\right\|_{L_{2, N}} \\
\leq & \sum_{j=1}^{J}\left\|\mu_{\theta}\left(j, x_{i}\right)-P\left(j \mid x_{i}, f_{0}\right)\right\|_{L_{2, N}}^{2} \\
+ & 2 \sum_{j=1}^{J} \eta_{N} \sqrt{R\left(\frac{\xi_{\min }(R)}{4 \zeta_{0}^{2}}\right)^{-1}}\left(\left\|\mu_{\widehat{\theta}}\left(j, x_{i}\right)-P\left(j \mid x_{i}, f_{0}\right)\right\|_{L_{2, N}}+\left\|\mu_{\theta}\left(j, x_{i}\right)-P\left(j \mid x_{i}, f_{0}\right)\right\|_{L_{2, N}}\right)
\end{aligned}
$$

by the Cauchy-Schwarz inequality, RCS, and the triangle inequality. Applying the inequality $2 x y \leq x^{2} a+y^{2} / a($ any $x, y, a>0)$ to $x=\eta_{N} \sqrt{R\left(\xi_{\min }(R) / 4 \zeta_{0}^{2}\right)^{-1}}$ and $y=\| \mu_{\widehat{\theta}}\left(j, x_{i}\right)-$ $P\left(j \mid x_{i}, f_{0}\right) \|_{L_{2, N}}$ and to $x=\eta_{N} \sqrt{R\left(\xi_{\min }(R) / 4 \zeta_{0}^{2}\right)^{-1}}$ and $y=\left\|\mu_{\theta}\left(j, x_{i}\right)-P\left(j \mid x_{i}, f_{0}\right)\right\|_{L_{2, N}}$, re- 
spectively, we obtain under $\prod_{j=1}^{J} E_{0, j} \cap E_{1, j}$,

$$
\begin{aligned}
& \sum_{j=1}^{J}\left\|\mu_{\widehat{\theta}}\left(j, x_{i}\right)-P\left(j \mid x_{i}, f_{0}\right)\right\|_{L_{2, N}}^{2} \\
\leq & \sum_{j=1}^{J}\left\|\mu_{\theta}\left(j, x_{i}\right)-P\left(j \mid x_{i}, f_{0}\right)\right\|_{L_{2, N}}^{2}+\sum_{j=1}^{J}\left\|\mu_{\widehat{\theta}}\left(j, x_{i}\right)-P\left(j \mid x_{i}, f_{0}\right)\right\|_{L_{2, N}}^{2} / a \\
& +\sum_{j=1}^{J}\left\|\mu_{\theta}\left(j, x_{i}\right)-P\left(j \mid x_{i}, f_{0}\right)\right\|_{L_{2, N}}^{2} / a+2 a \eta_{N}^{2} R\left(\xi_{\min }(R) / 4 \zeta_{0}^{2}\right)^{-1} \cdot J
\end{aligned}
$$

It follows that under $\bigcap_{j=1}^{J}\left(E_{0, j} \cap E_{1, j}\right)$, for all $a>1$,

$$
\sum_{j=1}^{J}\left\|\mu_{\widehat{\theta}}\left(j, x_{i}\right)-P\left(j \mid x_{i}, f_{0}\right)\right\|_{L_{2, N}}^{2} \leq \frac{a+1}{a-1} \sum_{j=1}^{J}\left\|\mu_{\theta}\left(j, x_{i}\right)-P\left(j \mid x_{i}, f_{0}\right)\right\|_{L_{2, N}}^{2}+\frac{\zeta_{0}^{2}}{\xi_{\min }(R)} \frac{8 a^{2}}{a-1} \eta_{N}^{2} R J
$$

The conclusion follows from

$$
\operatorname{Pr}\left\{\left(\bigcap_{j=1}^{J} E_{0, j}\right)^{C}\right\} \leq \sum_{j=1}^{J} \operatorname{Pr}\left\{E_{0, j}^{C}\right\}=J\left\{R \exp \left(-\frac{N \eta_{N}^{2} c_{0}^{2}}{8 \zeta_{0}^{2}}\right)+R \exp \left(-C_{1} \frac{N c_{0}^{4}}{\zeta_{0}^{4}}\right)\right\}
$$

by Lemma B.4 and $\operatorname{Pr}\left\{\left(\bigcap_{j=1}^{J} E_{1, j}\right)^{C}\right\}=J\left\{R \exp \left(-C_{1} \frac{N c_{0}^{4}}{\zeta_{0}^{4}}\right)+R^{2} \exp \left(-C_{2} \frac{N}{\zeta_{0}^{4} R^{2}}\right)\right\}$ by Lemma B.3.

\section{B.1 Proof of Theorem 4.1}

From Lemma B.5, we find that the best convergence rate will be obtained when the order of $\eta_{N}$ is as small as possible while keeping the convergence of $p_{N, R}$ to zero. By inspecting $p_{N, R}=$ $J\left\{R \exp \left(-\frac{N \eta_{N}^{2} c_{0}^{2}}{8 \zeta_{0}^{2}}\right)+2 R \exp \left(-C_{1} \frac{N c_{0}^{4}}{\zeta_{0}^{4}}\right)+R^{2} \exp \left(-C_{2} \frac{N}{\zeta_{0}^{2} R^{2}}\right)\right\}$, we note that the optimal rate is obtained when $\eta_{N}=O\left(\sqrt{\frac{\log (R(N) N)}{N}}\right)$ since the first term in $p_{N, R}$ dominates the second term in $p_{N, R}$ when $\eta_{N}$ is small enough and $p_{N, R} \rightarrow 0$ with $\eta_{N}=O\left(\sqrt{\frac{\log (R(N) N)}{N}}\right)$. The inspection of the third term in $p_{N, R}$ reveals that we also require $R=R(N)$ should satisfy $\frac{R(N)^{2} \log (R(N))}{N} \rightarrow 0$ so that $p_{N, R} \rightarrow 0$. 
The result of Theorem 4.1 follows from these requirements and Lemma B.5 as

$$
\begin{aligned}
& \frac{1}{J} \sum_{j=1}^{J}\left\|\mu_{\widehat{\theta}}\left(j, x_{i}\right)-P\left(j \mid x_{i}, f_{0}\right)\right\|_{L_{2, N}}^{2}= \\
& \quad O_{\mathbb{P}}\left(\max \left\{\frac{R(N) \log (R(N) N)}{N}, \frac{1}{J} \sum_{j=1}^{J}\left\|\mu_{\theta}\left(j, x_{i}\right)-P\left(j \mid x_{i}, f_{0}\right)\right\|_{L_{2, N}}\right\}\right)
\end{aligned}
$$

since $\frac{\zeta_{0}^{2}}{\xi_{\min }(R)} \frac{8 a^{2}}{a-1} \eta_{N}^{2} R(N)=O\left(\frac{\log (R(N) N)}{N} R(N)\right)$ under $\eta_{N}=O\left(\sqrt{\frac{\log (R(N) N)}{N}}\right)$ and because Lemma B.5 holds for any $\theta \in \Delta_{R(N)}$. Combining the rate conditions on $R(N)=C \cdot N^{\rho}$ with $\frac{K}{2 \bar{s}+K}<\rho$ and $\frac{R(N)^{2} \log R(N)}{N} \rightarrow 0$, we obtain $\frac{K}{2 \bar{s}+K}<\rho<1 / 2$. Also see that $\bar{s}>K / 2$ is required for $\rho$ to exist.

\section{B.2 Proof of Lemma 4.1}

First we construct approximating power series with the length of $L$ as $L$ different tensor products of higher order polynomials of $\beta_{(k)}$ 's in $\beta$

$$
\left\{\varphi_{1}(\beta), \ldots, \varphi_{l}(\beta), \ldots, \varphi_{L}(\beta)\right\}
$$

where $\varphi_{l}(\beta)$ is the $l^{\text {th }}$ element in the $L$ number of tensor product polynomials. The tensor products are defined by the functions $\varphi_{l}(\beta)=\beta_{(1)}^{l_{1}} \beta_{(2)}^{l_{2}} \cdots \beta_{(K)}^{l_{K}}$ with $\beta=\left(\beta_{(1)}, \beta_{(2)}, \ldots, \beta_{(K)}\right) \in$ $\mathcal{B}=\prod_{k=1}^{K}\left[\underline{\beta}_{(k)}, \bar{\beta}_{(k)}\right]$ and $l_{k}$ 's are exponents of each $\beta_{(k)}$. For example, we can let $\varphi_{1}(\beta)=1$, $\varphi_{2}(\beta)=\beta_{(1)}, \ldots$, and $\varphi_{l}(\beta)=\beta_{(1)}^{l_{1}} \beta_{(2)}^{l_{2}} \cdots \beta_{(K)}^{l_{K}}$.

Now note that $g_{j}(x, \beta) f(\beta)$ is a member of the Hölder class ( $\bar{s}$-smooth) of functions since it is uniformly bounded and all of its own and partial derivatives up to the order of $\bar{s}$ are also uniformly bounded by our restriction on $f$ in $\mathcal{H}^{(j)}$ and the logit specification assumption. Therefore, we can approximate $g_{j}(x, \beta) f(\beta)$ well using power series (see Chen, 2007) and obtain the approximation error rate due to Timan (1963) as

$$
\sup _{\beta \in \mathcal{B}}\left|g_{j}(x, \beta) f(\beta)-\sum_{l=1}^{L} a_{l}(x) \varphi_{l}(\beta)\right|=O\left(L^{-\bar{s} / K}\right)
$$


all $x \in \mathcal{X}$. Now define

$$
\underline{\beta}_{(k)}=b_{(k), 1}<b_{(k), 2}<\cdots<b_{(k), r_{j}+1}=\bar{\beta}_{(k)}, \quad k=1, \ldots, K
$$

be partitions of the intervals $\left[\underline{\beta}_{(k)}, \bar{\beta}_{(k)}\right], k=1, \ldots, K$, into $r_{1}, \ldots, r_{K}$ subintervals, respectively. Then we can define the $R=r_{1} r_{2} \cdots r_{K}$ number of subcubes

$$
C_{\iota_{1}, \ldots, \iota_{K}}=\prod_{k=1}^{K}\left[b_{(k), \iota_{k}}, b_{(k), \iota_{k}+1}\right], \iota_{k}=1,2, \ldots, r_{k},
$$

which become a partition $P(\mathcal{B})$ of $\mathcal{B}$. For any choice of $R$ points

$$
\left\{b_{\iota_{1}, \ldots, \iota_{K}} \in C_{\iota_{1}, \ldots, \iota_{K}} \mid \iota_{k}=1,2, \ldots, r_{k}, k=1, \ldots, K\right\}
$$

(one $b_{\iota_{1}, \ldots, \iota_{K}}$ for each of $R$ subcubes), now we can approximate a Riemann integral of $\sum_{l=1}^{L} a_{l}(x) \varphi_{l}(\beta)$ using a quadrature method with $R$ distinct weights

$$
\left\{c\left(\iota_{1}, \ldots, \iota_{K}\right) \equiv c\left(b_{\iota_{1}, \ldots, \iota_{K}}\right) \mid \iota_{k}=1, \ldots, r_{k}, k=1, \ldots, K\right\}
$$

such that

$$
\begin{aligned}
\int \sum_{l=1}^{L} a_{l}(x) \varphi_{l}(\beta) d \beta & =\sum_{l=1}^{L} a_{l}(x) \int \varphi_{l}(\beta) d \beta \\
& =\sum_{l=1}^{L} a_{l}(x) \sum_{C_{\iota_{1}, \ldots, \iota_{K}} \in P(\mathcal{B})} c\left(\iota_{1}, \ldots, \iota_{K}\right) \varphi_{k}\left(b_{\iota_{1}, \ldots, \iota_{K}}\right)+\mathcal{R}\left(\delta_{R}\right)
\end{aligned}
$$

where $\mathcal{R}\left(\delta_{R}\right)$ denotes a remainder term with $\delta_{R}=\max \left\{\operatorname{diam}\left(C_{\iota_{1}, \ldots, \iota_{K}}\right): C_{\iota_{1}, \ldots, \iota_{K}} \in P(\mathcal{B})\right\}$. Without loss of generality, we will pick $\delta_{R}=C \cdot R^{-1 / K}$. Noting that $\varphi_{l}(\beta)$ is a product of polynomials in $\beta_{(k)}$ 's by construction, we can apply Theorem 6.1.2 (Generalized Cartesian Product Rules) of Krommer and Ueberhuber (1998) and so we can approximate multivariate integrals with products of univariate integrals. Note that

$$
\int \varphi_{l}(\beta) d \beta=\prod_{k=1}^{K} \int \varphi_{l, k}\left(\beta_{(k)}\right) d \beta_{(k)}
$$

with $\varphi_{l}(\beta)=\sum_{k=1}^{K} \varphi_{l, k}\left(\beta_{(k)}\right)$. If we approximate $\int \varphi_{l, k}\left(\beta_{(k)}\right) d \beta_{(k)}$ using a univariate quadrature 
with weights $\left\{c_{k}(1), \ldots, c_{k}\left(r_{k}\right)\right\}$, we obtain

$$
\int \varphi_{l, k}\left(\beta_{(k)}\right) d \beta_{(k)}=\sum_{\iota_{k}=1}^{r_{k}} c_{k}\left(\iota_{k}\right) \varphi_{l, k}\left(b_{(k), \iota_{k}}\right)+\mathcal{R}_{l, k}\left(\delta_{R}\right)
$$

where $\mathcal{R}_{l, k}\left(\delta_{R}\right)$ denotes a remainder term. Then we can make the univariate quadrature become accurate (or exact) at least up to the order of $r_{k}$ (Theorem 5.2.1 in Krommer and Ueberhuber $(1998))$ i.e., $\int \beta_{(k)}^{p} d \beta_{(k)}=\sum_{\iota_{k}=1}^{r_{k}} c_{k}\left(\iota_{k}\right) b_{(k), \iota_{k}}^{p}$ with suitable choice of $c_{k}\left(\iota_{k}\right) \equiv c_{k}\left(b_{(k), \iota_{k}}\right)$ for all $p \leq r_{k}$.

For notational simplicity, we take $r_{k}=r_{1}$ for all $k$. Then with the $L=\left(r_{1}+1\right)^{K}=\left(R^{1 / K}+1\right)^{K}$ number of power series, we can include powers and cross products of $\beta_{(k)}$ 's at least up to the order of $r_{1}$. With the choice of $L=\left(r_{1}+1\right)^{K}$ and $c_{k}\left(\iota_{k}\right)$ 's that make the univariate quadrature exact at least up to the order of $r_{1}$, we can let

$$
\begin{aligned}
\int \varphi_{l}(\beta) d \beta & =\prod_{k=1}^{K} \int \varphi_{l, k}\left(\beta_{(k)}\right) d \beta_{(k)} \\
& =\prod_{k=1}^{K}\left(\sum_{\iota_{k}=1}^{r_{1}} c_{k}\left(\iota_{k}\right) \varphi_{l, k}\left(b_{(k), \iota_{k}}\right)\right)
\end{aligned}
$$

for $l=1, \ldots, L$. By adding and subtracting terms, it follows that

$$
\begin{aligned}
& P(j \mid x, f)-\mu_{\theta^{*}}(j, x)= \\
& \int f(\beta) g_{j}(x, \beta) d \beta-\int \sum_{l=1}^{L} a_{l}(x) \varphi_{l}(\beta) d \beta \\
& +\sum_{l=1}^{L} a_{l}(x) \int \varphi_{l}(\beta) d \beta-\sum_{l=1}^{L} a_{l}(x) \prod_{k=1}^{K}\left(\sum_{\iota_{k}=1}^{r_{k}} c_{k}\left(\iota_{k}\right) \varphi_{l, k}\left(b_{(k), \iota_{k}}\right)\right) \\
& +\sum_{l=1}^{L} a_{l}(x) \prod_{k=1}^{K}\left(\sum_{\iota_{k}=1}^{r_{k}} c_{k}\left(\iota_{k}\right) \varphi_{l, k}\left(b_{(k), \iota_{k}}\right)\right)-\mu_{\theta^{*}}(j, x) .
\end{aligned}
$$

We first bound the terms in (36) as

$$
\begin{aligned}
& \left|\int f(\beta) g_{j}(x, \beta) d \beta-\int \sum_{l=1}^{L} a_{l}(x) \varphi_{l}(\beta) d \beta\right| \\
\leq & \int\left|f(\beta) g_{j}(x, \beta)-\sum_{l=1}^{L} a_{l}(x) \varphi_{l}(\beta)\right| d \beta=O\left(L^{-\bar{s} / K} \cdot \operatorname{vol}(\mathcal{B})\right)
\end{aligned}
$$


from (34). Second note that (37) becomes zero due to (35).

Now we construct $\widetilde{\varphi}_{l}\left(b^{r}\right) r=1, \ldots, R$ such that

$$
\sum_{r=1}^{R} \widetilde{\varphi}_{l}\left(b^{r}\right)=\prod_{k=1}^{K}\left\{\sum_{\iota_{k}=1}^{r_{k}} c_{k}\left(\iota_{k}\right) \varphi_{l, k}\left(b_{(k), \iota_{k}}\right)\right\}
$$

with $R=r_{1} \cdots r_{K}$ and $b^{r}=\left(b_{(1)}^{r}, b_{(2),}^{r}, \ldots, b_{(K)}^{r}\right)$ in $\mathbf{b} \equiv\left\{b: b=\left(b_{(1), \iota_{1}}, \ldots, b_{(K), \iota_{K}}\right), \iota_{1}=\right.$ $\left.1, \ldots, r_{1}, \ldots, \iota_{K}=1, \ldots, r_{K}\right\}$. Then, we have

$$
\widetilde{\varphi}_{l}\left(b^{r}\right)=c_{1}\left(b_{(1), \iota_{1}}^{r}\right) \cdots c_{K}\left(b_{(K), \iota_{K}}^{r}\right) \varphi_{l}\left(b^{r}\right)
$$

for any $b^{r}=\left(b_{(1), \iota_{1}}, \ldots, b_{(K), \iota_{K}}\right)$ in $\mathbf{b}, r=1, \ldots, R=r_{1} r_{2} \cdots r_{K}$. Define

$$
\theta^{* r}=\frac{c_{1}\left(b_{(1), \iota_{1}}^{r}\right) \cdots c_{K}\left(b_{(K), \iota_{K}}^{r}\right) f\left(b^{r}\right)}{\sum_{r=1}^{R} c_{1}\left(b_{(1), \iota_{1}}^{r}\right) \cdots c_{K}\left(b_{(K), \iota_{K}}^{r}\right) f\left(b^{r}\right)} .
$$

It follows that

$$
\begin{aligned}
& \left|\sum_{l=1}^{L} a_{l}(x) \widetilde{\varphi}_{l}\left(b^{r}\right)-\theta^{* r} \gamma\left(r, j, x_{j}\right)\right| \\
& =\left|\begin{array}{c}
\sum_{l=1}^{L} a_{l}(x) c_{1}\left(b_{(1), \iota_{1}}^{r}\right) \cdots c_{K}\left(b_{(K), \iota_{K}}^{r}\right) \varphi_{l}\left(b^{r}\right) \\
-\frac{c_{1}\left(b_{(1), \iota_{1}}^{r}\right) \cdots c_{K}\left(b_{(K), \iota_{K}}^{r}\right) f\left(b^{r}\right)}{\sum_{r=1}^{R} c_{1}\left(b_{(1), \iota_{1}}^{r}\right) \cdots c_{K}\left(b_{(K), \iota_{K}}^{r}\right) f\left(b^{r}\right)} \gamma\left(r, j, x_{j}\right)
\end{array}\right| \\
& =\left|c_{1}\left(b_{(1), \iota_{1}}^{r}\right) \cdots c_{K}\left(b_{(K), \iota_{K}}^{r}\right)\right|\left|\sum_{l=1}^{L} a_{l}(x) \varphi_{l}\left(b^{r}\right)-\frac{f\left(b^{r}\right)}{\sum_{r=1}^{R} c_{1}\left(b_{(1), \iota_{1}}^{r}\right) \cdots c_{K}\left(b_{(K), \iota_{K}}^{r}\right) f\left(b^{r}\right)} \gamma\left(r, j, x_{j}\right)\right| \\
& \leq\left|c_{1}\left(b_{(1), \iota_{1}}^{r}\right) \cdots c_{K}\left(b_{(K), \iota_{K}}^{r}\right)\right|\left|\sum_{l=1}^{L} a_{l}(x) \varphi_{l}\left(b^{r}\right)-f\left(b^{r}\right) \gamma\left(r, j, x_{j}\right)\right| \\
& +\left|c_{1}\left(b_{(1), \iota_{1}}^{r}\right) \cdots c_{K}\left(b_{(K), \iota_{K}}^{r}\right)\right|\left|\frac{\sum_{r=1}^{R} c_{1}\left(b_{(1), \iota_{1}}^{r}\right) \cdots c_{K}\left(b_{(K), \iota_{K}}^{r}\right) f\left(b^{r}\right)-1}{\sum_{r=1}^{R} c_{1}\left(b_{(1), \iota_{1}}^{r}\right) \cdots c_{K}\left(b_{(K), \iota_{K}}^{r}\right) f\left(b^{r}\right)}\right|\left|f\left(b^{r}\right) \gamma\left(r, j, x_{j}\right)\right| \\
& =O\left(R^{-1} L^{-\bar{s} / K}\right),
\end{aligned}
$$

First note that $\left|c_{1}\left(b_{(1), \iota_{1}}^{r}\right) \cdots c_{K}\left(b_{(K), \iota_{K}}^{r}\right)\right|$ is at most $O\left(R^{-1}\right)$, which is obtained if one uses the uniform weights, i.e., $c_{k}\left(b_{(k), \iota_{k}}^{r}\right)=\ldots=c_{k}\left(b_{(k), \iota_{k}}^{R}\right)$ for $k=1, \ldots, K$. Second note that $\left|\sum_{l=1}^{L} a_{l}(x) \varphi_{l}\left(b^{r}\right)-f\left(b^{r}\right) \gamma\left(r, j, x_{j}\right)\right|=O\left(L^{-\bar{s} / K}\right)$ due to the bound in (34). Third note that 
$\sum_{r=1}^{R} c_{1}\left(b_{(1), \iota_{1}}^{r}\right) \cdots c_{K}\left(b_{(K), \iota_{K}}^{r}\right) f\left(b^{r}\right)$ is another quadrature approximation of integral $\int_{\mathcal{B}} f(\beta) d \beta=$ 1. Since $f(\beta)$ itself belongs to a Hölder class, the approximation error rate of this integral becomes $O\left(L^{-\bar{s} / K}\right)$. Combining these results, our conclusion of the bound in (39) follows.

Now by letting $\theta^{*}=\left(\theta^{* 1}, \ldots, \theta^{* R}\right)$, we have

$$
\begin{aligned}
& \left|\sum_{l=1}^{L} a_{l}(x) \prod_{k=1}^{K}\left\{\sum_{\iota_{k}=1}^{r_{k}} c_{k}\left(\iota_{k}\right) \varphi_{l, k}\left(b_{(k), \iota_{k}}\right)\right\}-\mu_{\theta^{*}}(j, x)\right| \\
= & \left|\sum_{r=1}^{R} \sum_{l=1}^{L} a_{l}(x) \widetilde{\varphi}_{l}\left(b^{r}\right)-\sum_{r=1}^{R} \theta^{* r} \gamma\left(r, j, x_{j}\right)\right| \\
\leq & \sum_{r=1}^{R}\left|\sum_{l=1}^{L} a_{l}(x) \widetilde{\varphi}_{l}\left(b^{r}\right)-\theta^{* r} \gamma\left(r, j, x_{j}\right)\right| \\
= & O\left(R R^{-1} L^{-\bar{s} / K}\right)=O\left(L^{-\bar{s} / K}\right)
\end{aligned}
$$

where the second equality holds by (39). From this, we bound (38) as $O\left(L^{-\bar{s} / K}\right)$.

Combining the bounds, we conclude

$$
\begin{aligned}
\left|P(j \mid x, f)-\mu_{\theta^{*}}(j, x)\right| & =O\left(L^{-\bar{s} / K} \cdot \operatorname{vol}(\mathcal{B})\right)+0+O\left(L^{-\bar{s} / K}\right)=O\left(L^{-\bar{s} / K}\right) \\
& =O\left(\left(\left(R^{1 / K}+1\right)^{K}\right)^{-\bar{s} / K}\right)=O\left(\left(R^{1 / K}+1\right)^{-\bar{s}}\right) \leq O\left(R^{-\bar{s} / K}\right) .
\end{aligned}
$$

The second conclusion in the lemma is trivial since the above holds for all $x \in \mathcal{X}$.

\section{B.3 Proof of Lemma 4.2}

Define

$$
\begin{aligned}
\hat{A}(x) & =\frac{1}{R} \sum_{r=1}^{R} f\left(\beta^{r}\right) \gamma(r, j, x), A(x)=\frac{1}{\prod_{k=1}^{K}\left(\bar{\beta}_{(k)}-\underline{\beta}_{(k)}\right)} \int_{B} f(\beta) \frac{\exp \left(x_{j, i}^{\prime} \beta\right)}{1+\sum_{j^{\prime}=1}^{J} \exp \left(x_{j^{\prime}, i}^{\prime} \beta\right)} d \beta \\
\hat{B} & =(1 / R) \sum_{r=1}^{R} f\left(\beta^{r}\right), B=\frac{1}{\prod_{k=1}^{K}\left(\bar{\beta}_{(k)}-\underline{\beta}_{(k)}\right)} \int_{B} f(\beta) d \beta .
\end{aligned}
$$


Then, by plugging in definition of terms and adding and subtracting terms, we have

$$
\begin{aligned}
\sqrt{R}\left(\mu_{\theta^{*}}(j, x)-P(j \mid x, f)\right) & =\sqrt{R}(\hat{A}(x) / \hat{B}-A(x) / B) \\
& =\sqrt{R} \frac{(\hat{A}(x)-A(x))}{\hat{B}}-\sqrt{R} \frac{A(x)}{B \hat{B}}(\hat{B}-B) .
\end{aligned}
$$

Applying the Lindeberg-Levy central limit theorem and the law of large numbers, we further obtain

$$
\begin{aligned}
\sqrt{R}(\hat{A}(x)-A(x)) & =O_{\mathbb{P}}(1) \\
\hat{A}(x) & =A(x)+o_{\mathbb{P}}(1) \\
\hat{B} & =B+o_{\mathbb{P}}(1) \\
\sqrt{R}(\hat{B}-B) & =O_{\mathbb{P}}(1)
\end{aligned}
$$

uniformly over $x \in \mathcal{X}$ since $\frac{\exp \left(x_{j, i}^{\prime} \beta\right)}{1+\sum_{j^{\prime}=1}^{J} \exp \left(x_{j^{\prime}, i}^{\prime} \beta\right)}$ is uniformly bounded. Combining (40) and (41), we obtain $\sqrt{R}\left(\mu_{\theta^{*}}(j, x)-P(j \mid x, f)\right)=O_{\mathbb{P}}(1)$.

The second claim in Lemma 4.2 follows by applying the dominated convergence theorem, noting that $\hat{A}(x)$ is uniformly bounded by $M \geq \sup _{\beta \in \mathcal{B}}|f(\beta)|$.

\section{B.4 Proof of Theorem 4.2}

The first result of Theorem 4.2 follows from Theorem 4.1 combined with Lemma 4.1 with $R(N)$ satisfying (17) so that the variance term and the bias term are balanced. Now we show the second claim. Note that with probability approaching to one, we have $2\left\|g_{j}\left(\cdot, \beta^{r}\right)\right\|_{L_{2, N}} \geq\left\|g_{j}\left(\cdot, \beta^{r}\right)\right\|_{L_{2}} \geq$ $c_{0}>0$ for all $r=1, \ldots, R$ and $j=1, \ldots, J$ by Assumption 4.2(ii) and Lemma B.1. It follows 
that

$$
\begin{aligned}
& \frac{c_{0}}{2} \sum_{r=1}^{R}\left|\widehat{\theta}^{r}-\theta_{0}^{* r}\right| \\
& \leq \frac{1}{J} \sum_{j=1}^{J} \sum_{r=1}^{R}\left\|g_{j}\left(\cdot, \beta^{r}\right)\right\|_{L_{2, N}}\left|\widehat{\theta}^{r}-\theta_{0}^{* r}\right| \leq \frac{1}{J} \sum_{j=1}^{J} \sqrt{R} \sqrt{\sum_{r=1}^{R}\left\|g_{j}\left(\cdot, \beta^{r}\right)\right\|_{L_{2, N}}^{2}\left(\widehat{\theta}^{r}-\theta_{0}^{* r}\right)^{2}} \\
& \leq \sqrt{\frac{\xi_{\min }(R)}{4 \zeta_{0}^{2}}} \sqrt{R} \frac{1}{J} \sum_{j=1}^{J}\left\|\mu_{\widehat{\theta}}\left(j, x_{i}\right)-\mu_{\theta_{0}^{*}}\left(j, x_{i}\right)\right\|_{L_{2, N}} \\
& \leq \sqrt{\frac{\xi_{\min }(R)}{4 \zeta_{0}^{2}}} \sqrt{R} \frac{1}{J} \sum_{j=1}^{J}\left(\left\|\mu_{\widehat{\theta}}\left(j, x_{i}\right)-P\left(j \mid x_{i}, f_{0}\right)\right\|_{L_{2, N}}+\left\|\mu_{\theta_{0}^{*}}\left(j, x_{i}\right)-P\left(j \mid x_{i}, f_{0}\right)\right\|_{L_{2, N}}\right) \\
& \leq \sqrt{\frac{\xi_{\min }(R)}{4 \zeta_{0}^{2}}} \sqrt{R}\left(\sqrt{\frac{1}{J} \sum_{j=1}^{J}\left\|\mu_{\widehat{\theta}}(j, \cdot)-P\left(j \mid \cdot, f_{0}\right)\right\|_{L_{2, N}}^{2}}+\sqrt{\frac{1}{J} \sum_{j=1}^{J}\left\|\mu_{\theta_{0}^{*}}(j, \cdot)-P\left(j \mid \cdot, f_{0}\right)\right\|_{L_{2, N}}^{2}}\right)
\end{aligned}
$$

where the second inequality holds by RCS, the third inequality holds similarly with (33), the fourth inequality holds by the triangle inequality, and the last inequality is due to the CauchySchwarz inequality. Therefore, from Lemma 4.1 and the first result of Theorem 4.2 with our choice of $R(N)$ and $\eta_{N}=O\left(\sqrt{\frac{\log (R(N) N)}{N}}\right)$ (so that the variance term balances with the bias term), it follows that

$$
\sum_{r=1}^{R}\left|\widehat{\theta}^{r}-\theta_{0}^{* r}\right|=O\left(\sqrt{R(N)} \sqrt{\frac{R(N) \cdot \log (R(N) N)}{N}}\right)=O\left(R(N) \sqrt{\frac{\log (R(N) N)}{N}}\right) .
$$

\section{B.5 Proof of Theorem 4.3}

Define a pseudo true distribution such that $F_{R}(\beta)=\sum_{r=1}^{R} \theta_{0}^{* r} 1\left[\beta^{r} \leq \beta\right]$. It is not difficult to see that

$$
\begin{aligned}
\sup _{\beta \in \mathcal{B}_{0}}\left|\widehat{F}(\beta)-F_{R}(\beta)\right| & =\sup _{\beta \in \mathcal{B}_{0}}\left|\sum_{r=1}^{R} \widehat{\theta}^{r} 1\left[\beta^{r} \leq \beta\right]-\sum_{r=1}^{R} \theta_{0}^{* r} 1\left[\beta^{r} \leq \beta\right]\right| \\
& =\sup _{\beta \in \mathcal{B}_{0}}\left|\sum_{r=1}^{R}\left(\widehat{\theta}^{r}-\theta_{0}^{* r}\right) 1\left[\beta^{r} \leq \beta\right]\right| \\
& \leq \sum_{r=1}^{R}\left|\widehat{\theta}^{r}-\theta_{0}^{* r}\right|=O_{\mathbb{P}}\left(R(N) \sqrt{\frac{\log (R(N) N)}{N}}\right)
\end{aligned}
$$


where the last inequality holds by the triangle inequality and the last equality holds by Theorem 4.2. Note that

$$
F_{0}(\beta)=\int f_{0}(b) 1[b \leq \beta] d b
$$

and $F_{R}(\beta)$ becomes a quadrature approximation. We use a similar strategy with the proof of Lemma 4.1 where we approximate $f(b)$ with $\sum_{l=1}^{L} a_{f, l} \varphi_{l}(b)$ such that $\sup _{\beta \in \mathcal{B}_{0}}\left|f(b)-\sum_{l=1}^{L} a_{f, l} \varphi_{l}(b)\right|=$ $O\left(L^{-\bar{s} / K}\right)$ due to Timan (1963). We find

$$
F_{0}(\beta)-F_{R}(\beta)=O\left(R^{-\bar{s} / K}\right) \text { a.e. } \beta \in \mathcal{B}_{0}
$$

We conclude

$$
\begin{aligned}
\left|\widehat{F}_{N}(\beta)-F_{0}(\beta)\right| & \leq\left|\widehat{F}_{N}(\beta)-F_{R}(\beta)\right|+\left|F_{R}(\beta)-F_{0}(\beta)\right| \\
& =O_{\mathbb{P}}\left(R(N) \sqrt{\frac{\log (R(N) N)}{N}}\right)+O\left(R^{-\bar{s} / K}\right) \text { a.e. } \beta \in \mathcal{B}_{0} .
\end{aligned}
$$




\section{References}

[1] Ackerberg, Daniel A. (2009). "A New Use of Importance Sampling to Reduce Computational Burden in Simulation Estimation." Quantitative Marketing and Economics.

[2] Akhiezer, N.I. (1965), The classical moment problem and some related questions in analysis, Oliver \& Boyd.

[3] Aliprantis, C.D. and K.C. Border (2006), Infinite Dimensional Analysis: A Hitchhiker's Guide, Springer.

[4] Amemiya, T. (1983), "Non-Linear Regression Models," Handbook of Econometrics I, edited by Z. Griliches and M.D. Intrilligator, 333-389.

[5] Andrews, D.K.W. (1994), "Empirical Process Methods in Econometrics," Handbook of Econometrics IV, edited by R.F. Engle and D.L. McFadden, 2247-2294.

[6] Andrews, D.K.W. (2000), "'Inconsistency of the Bootstrap When a Parameter Is on the Boundary of the Parameter Space," Econometrica, 68, 399-405.

[7] Andrews D.K.W. (1999), "Estimation When a Parameter is on a Boundary," Econometrica 67, 1341-1383.

[8] Andrews D.K.W. (2002), "Generalized Method of Moments Estimation When a Parameter is on a Boundary," Journal of Business 8 Economics Statistics 20-4, 530-544.

[9] Andrews D.K.W and P. Guggenberger (2009a), "Hybrid and Size-corrected Subsample Methods," Econometrica, 77.

[10] Andrews, D.K.W and P. Guggenberger (2009b), "Applications of Subsampling, Hybrid, and Size-Correction Methods," Yale working paper.

[11] Andrews, D.K.W and P. Guggenberger (2010), "Asymptotic Size and a Problem with Subsampling and the m Out of n Bootstrap", Econometric Theory, 26.

[12] Bajari, Patrick, Jeremy T. Fox, Kyoo il Kim and Stephen P. Ryan, "The Random Coefficients Logit Model Is Identified", NBER working paper.

[13] Baraud, Y. (2002), "Model Selection for Regression on a Random Design", ESAIM Probability \& Statistics \%, 127-146 
[14] Berry, S and Haile, P. (2008), "Nonparametric Identification of Multinomial Choice Models with Heterogeneous Consumers and Endogeneity", working paper.

[15] Berry, S., J. Levinsohn, and A. Pakes (1995), "Automobile Price in Market Equilibrium", Econometrica (63), July 1995.

[16] Biernacki, Christophe, Gilles Celeux and Gérard Govaert (2003), "Choosing starting values for the EM algorithm for getting the highest likelihood in multivariate Gaussian mixture models," Computational Statistics $\&$ Data Analysis, 41, 561-575.

[17] Billingsley, Patrick (1995), Probability and Measure, 3rd Edition.

[18] Böhning, D. "Convergence of Simar's Algorithm for Finding the Maximum Likelihood Estimate of a Compound Poisson Process", The Annals of Statistics, 10(3), 1006-1008. 1982.

[19] Briesch, R.A., P.K. Chintagunta, and R.L. Matzkin (2007), "Nonparametric Discrete Choice Models with Unobserved Heterogeneity", SMU working paper.

[20] Burda, Martin, Matthew Harding and Jerry Hausman (2008), "A Bayesian Mixed LogitProbit for Multinomial Choice Demand Models", University of Toronto working paper.

[21] Chen, X. (2007), "Large Sample Sieve Estimation of Semi-Nonparametric Models," Handbook of Econometrics VI, Elsevier.

[22] Chen, X. and D. Pouzo (2009a), "Estimation of Nonparametric Conditional Moment Models With Possibly Nonsmooth Moments", Yale working paper.

[23] Chen, X. and D. Pouzo (2009b), "Efficient Estimation of Semiparametric Conditional Moment Models with Possibly Nonsmooth Residuals", Journal of Econometrics, forthcoming.

[24] Chiappori, Pierre André and Ivana Komunjer, "On the Nonparametric Identification of Multiple Choice Models", UCSD working paper.

[25] Cramér, H. and H. Wold (1936), "Some Theorems on Distribution Functions", Journal of the London Mathematical Society, s1-11(4), 290-294.

[26] Dempster, A.P., N.M. Laird and D.B. Rubin (1977), "Maximum likelihood from incomplete data via the EM algorithm", Journal of the Royal Statistical Society, 39, 1, 1-38. 
[27] D'Haultfoeuille, Xavier (2009), "On the Completeness Condition in Nonparametric Instrumental Problems", Econometric Theory.

[28] Devroye, Luc, and Laszlo Gyorfi (1985), Nonparametric Density Estimation, The $L_{1}$ View, New York, Wiley.

[29] Duflo, Esther, Rema Hanna and Stephen P. Ryan (2008), "Monitoring Works: Getting Teachers to Come to School", MIT working paper.

[30] Fox, Jeremy T. and Amit Gandhi (2009a), "Identifying Heterogeneity in Economic Choice Models", NBER working paper.

[31] Fox, Jeremy T. and Amit Gandhi (2009b), "Full Identification in the Generalized Selection Model", University of Chicago working paper.

[32] Gautier, Eric and Kitamura, Yuichi. (2008), "Nonparametric Estimation in Random Coefficients Binary Choice Models", CREST working paper.

[33] Geweke, J. (1986), "Exact Inference in the Inequality Constrained Normal Linear Regression Model," Journal of Applied Econometrics, Vol. 1, No. 2, pp. 127-141.

[34] Golub, GH, M. Heath and G. Wahba (1979), "Generalized cross-validation as a method for choosing a good ridge parameter," Technometrics, 215-223.

[35] Heckman, J. and Singer, B. "Method for Minimizing the Impact of Distributional Assumptions in Econometric Models for Duration Data”, Econometrica 52(2), 271-320 .

[36] Hoderlein, Stefan, Jussi Klemelä and Enno Mammen (2008), "Analyzing the Random Coefficient Model Nonparametrically", Brown University working paper.

[37] Hoeffding, W. (1963), "Probability Inequalities for Sums of Independent Random Variables", Journal of the American Statistical Association, 58, 13-30.

[38] Huber, J. (2004) Robust Statistics, Wiley.

[39] Ichimura, Hidehiko and T. Scott Thompson (1998), "Maximum likelihood estimation of a binary choice model with random coefficients of unknown distribution," Journal of Econometrics, 86(2), 269-295.

[40] Judd, K. L. (1998), Numerical Methods in Economics. MIT Press. 
[41] Judge, G.G. and Takayama, T. (1966), "Inequality Restrictions in Regression Analysis", Journal of the American Statistical Association, Vol. 61, No. 313, pp. 166-181.

[42] Kamakura, W.A. (1991), "Estimating flexible distributions of ideal-points with external analysis of preferences", Psychometrika, 56, 3, 419-431.

[43] Karlis, Dimitris and Evdokia Xekalaki (2003), "Choosing initial values for the EM algorithm for finite mixtures", Computational Statistics \& Data Analysis, 41, 577-590.

[44] Krein, M.G. and A.A. Nudel'man (1973, translation 1977), The Markov moment problem and extremal problems: ideas and problems of P. L. Čebyšev and A. A. Markov and their further development. Translations of mathematical monographs v. 50, American Mathematical Society, Providence.

[45] Krommer, A.R. and C.W. Ueberhuber (1998), Computation Integration, SIAM.

[46] Laird, Nan (1978), "Nonparametric Maximum Likelihood Estimation of a Mixing Distribution", Journal of the American Statistical Association, Vol. 73, No. 364, pp. 805-811.

[47] Lehmamm, E.L. and J.P. Romano (2005), Testing Statistical Hypotheses, 3rd Ed. Springer.

[48] Lewbel, Arthur. (2000) "Semiparametric qualitative response model estimation with unknown heteroscedasticity or instrumental variables", Journal of Econometrics, 97, 1, 145177.

[49] Li, Jonathan Q. and Andrew R. Barron (2000), "Mixture density estimation", Advances in Neural Information Processing Systems, Vol. 12, pp. 279-285.

[50] Liew, C.K. (1976), "Inequality Constrained Least-Squares Estimation", Journal of the American Statistical Association, Vol. 71, No. 355, pp. 746-751.

[51] Lindsay, B.G. (1983) "The Geometry of Mixture Likelihoods: A General Theory", The Annals of Statistics, 11(1), 86-94.

[52] Manski, Charles F. (1975) "Maximum Score Estimation of the Stochastic Model of Choice", Journal of Econometrics, 3(3), 205-228.

[53] McFadden, Daniel and Kenneth Train (2000), "Mixed MNL models for discrete response", Journal of Applied Econometrics, 15(5): 447-470. 
[54] McLachlan, G.J. and D. Peel (2000), Finite Mixture Models. Wiley.

[55] Nevo, Aviv. 2001, "Measuring Market Power in the Ready-to-Eat Cereal Industry", Econometrica, 69(2): 307-342.

[56] Newey, W.K. (1997), "Convergence Rates and Asymptotic Normality for Series Estimators", Journal of Econometrics 79, 147-168.

[57] Parthasarathy, K.R. (1967), Probability Measures on Metric Spaces, Academic Press.

[58] Petrin, Amil (2002), "Quantifying the Benefits of New Products: The Case of the Minivan", Journal of Political Economy, 110:705-729, 2002.

[59] Petrin, Amil and Kenneth Train (2009), "Control Function Corrections for Omitted Attributes in Differentiated Products Markets", Journal of Marketing Research.

[60] Pilla, Ramani S. and Bruce G. Lindsay (2001), "Alternative EM methods for nonparametric finite mixture models", Biometrika, 88, 2, 535-550.

[61] Quandt, R.E. and Ramsey, J.B. (1978), "Estimating Mixtures of Normal Distributions and Switching Regressions," Journal of the American Statistical Association, 73, 364, 730-738.

[62] Rossi, Peter E., Greg M. Allenby, and Robert McCulloch (2005), Bayesian Statistics and Marketing. West Sussex: John Willy \& Sons.

[63] Rust, John (1987), "Optimal Replacement of GMC Bus Engines: An Empirical Model of Harold Zurcher", Econometrica, 55(5): 999-1033.

[64] Rust, John (1994), "Structural Estimation of Markov Decision Processes", in Handbook of Econometrics, vol. 4, edited by Robert F. Engle and Daniel L. McFadden. Amsterdam: North-Holland.

[65] Rust, John (1997), "Using randomization to break the curse of dimensionality", Econometrica, 65, 3, 487-516.

[66] Seidel, Wilfried, Karl Mosler and Manfred Alker (2000), "A cautionary note on likelihood ratio tests in mixture models", Annals of the Institute of Statistical Mathematics, 52, 3, 418-487, 
[67] Shohat, J.A. and Tamarkin, J.D. (1943), The Problem of Moments, American Mathematics Society, Providence, RI.

[68] Teicher, H. (1963), "Identifiability of Finite Mixtures", Annals of Mathematical Statistics, 34, 1265-1269.

[69] Tibshirani, R. (1996), "Regression shrinkage and selection via the Lasso", Journal of the Royal Statistical Society, Series B. 58, 267-288.

[70] Timan, A.F. (1963), Theory of Approximation of Functions of a Real Variable, MacMilan, New York.

[71] Van der Vaart, W. and J.A. Wellner (1996), Weak Convergence and Empirical Processes, Springer Series in Statistics, New York.

[72] Verbeek, J.J., N. Vlassis and B. Kröse (2003), "Efficient Greedy Learning of Gaussian Mixture Models", Neural Computation, Vol. 15, pp 469-485.

[73] Wolak, F. "Testing inequality constraints in linear econometric models", Journal of Econometrics, Elsevier, vol. 41(2), pages 205-235, June 1989.

[74] Yakowitz, S.J. and Spragins, J.D. (1968) "On the identifiability of finite mixtures", The Annals of Mathematical Statistics, pages 209-214.

[75] Zolotarev, V.M. (2001), "Lévy-Prokhorov metric", Encyclopaedia of Mathematics, Hazewinkel, M. (Ed.), Kluwer. 
Figure 1: True, Estimated, and 95\% Sampling Intervals for Marginal Densities from the Monte Carlo Experiment

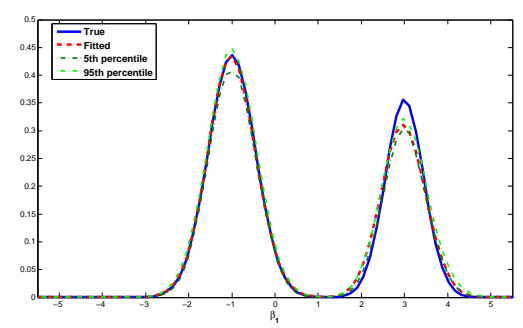

Density of $\beta_{1}$ : Two Normal Mixtures

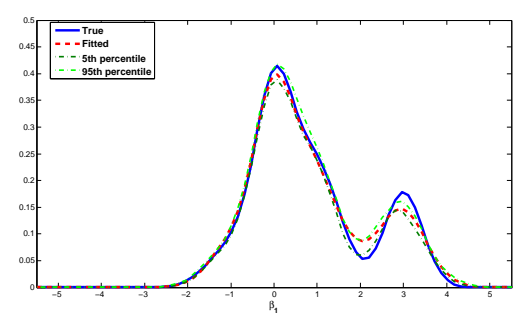

Density of $\beta_{1}$ : Four Normal Mixtures

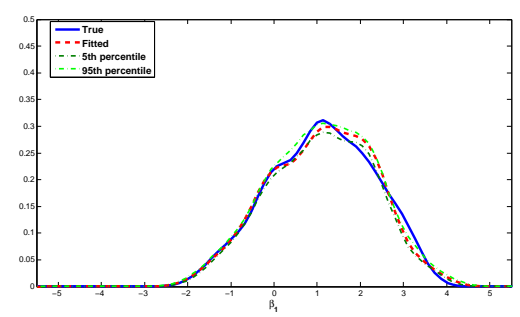

Density of $\beta_{1}$ : Six Normal Mixtures

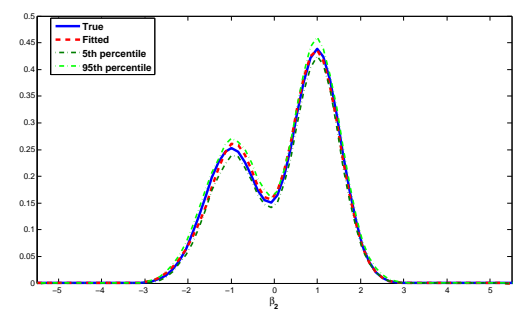

Density of $\beta_{2}$ : Two Normal Mixtures

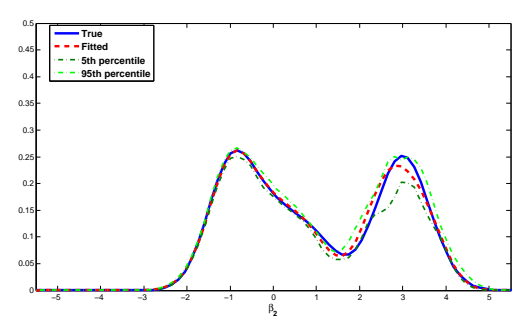

Density of $\beta_{2}$ : Four Normal Mixtures

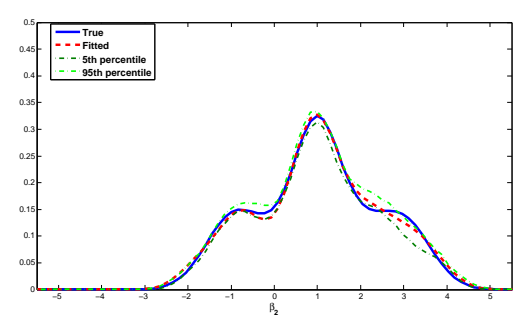

Density of $\beta_{2}$ : Six Normal Mixtures 
Figure 2: True and Estimated Joint Densities from the Monte Carlo Experiment

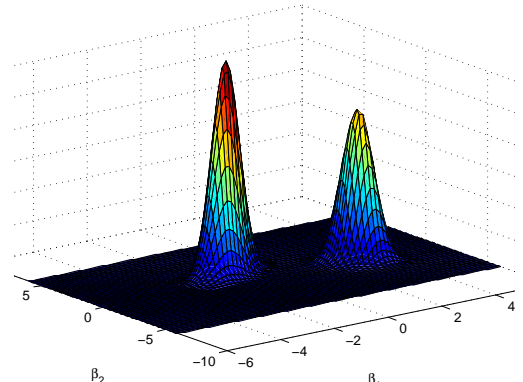

Two Normal Mixtures: Truth

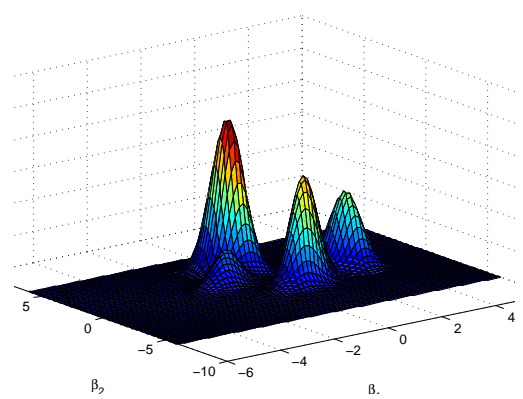

Four Normal Mixtures: Truth

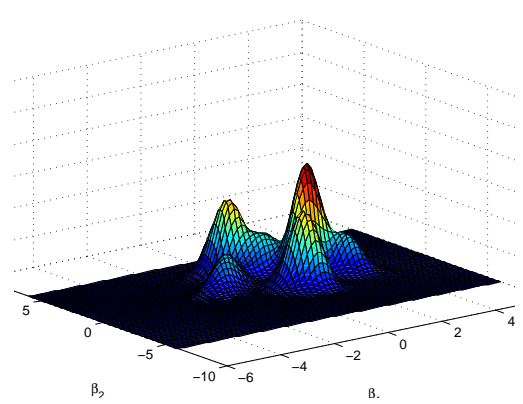

Six Normal Mixtures: Truth

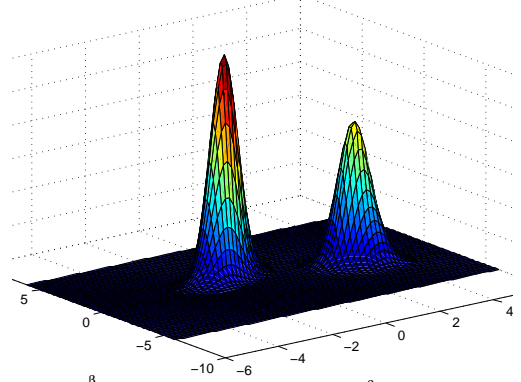

Two Normal Mixtures: Estimated

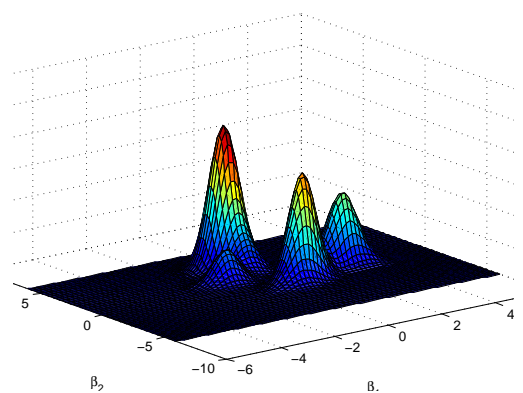

Four Normal Mixtures: Estimated

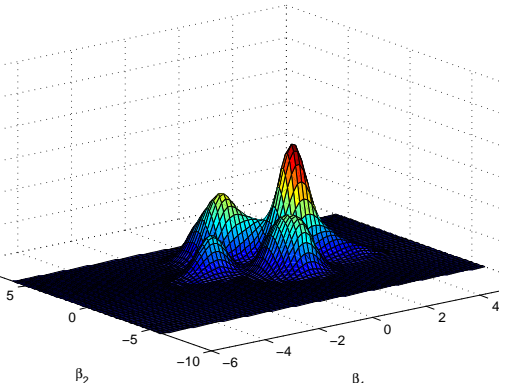

Six Normal Mixtures: Estimated 
Figure 3: Estimated Distributions of Heterogeneity for the Benefits of Staying Home
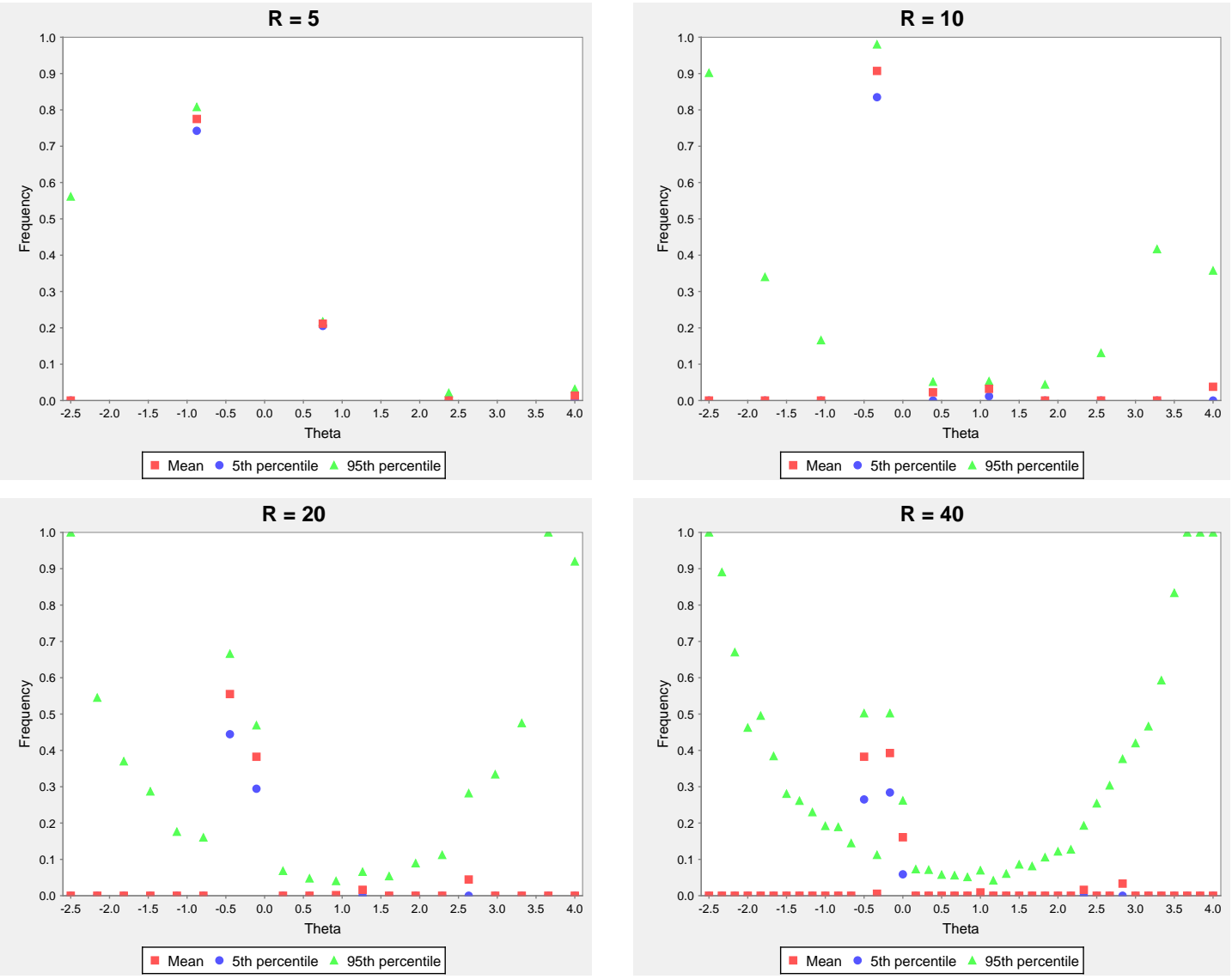
Figure 4: Predicted Distribution of Days Worked in Months Where First Five Days Used for Estimation
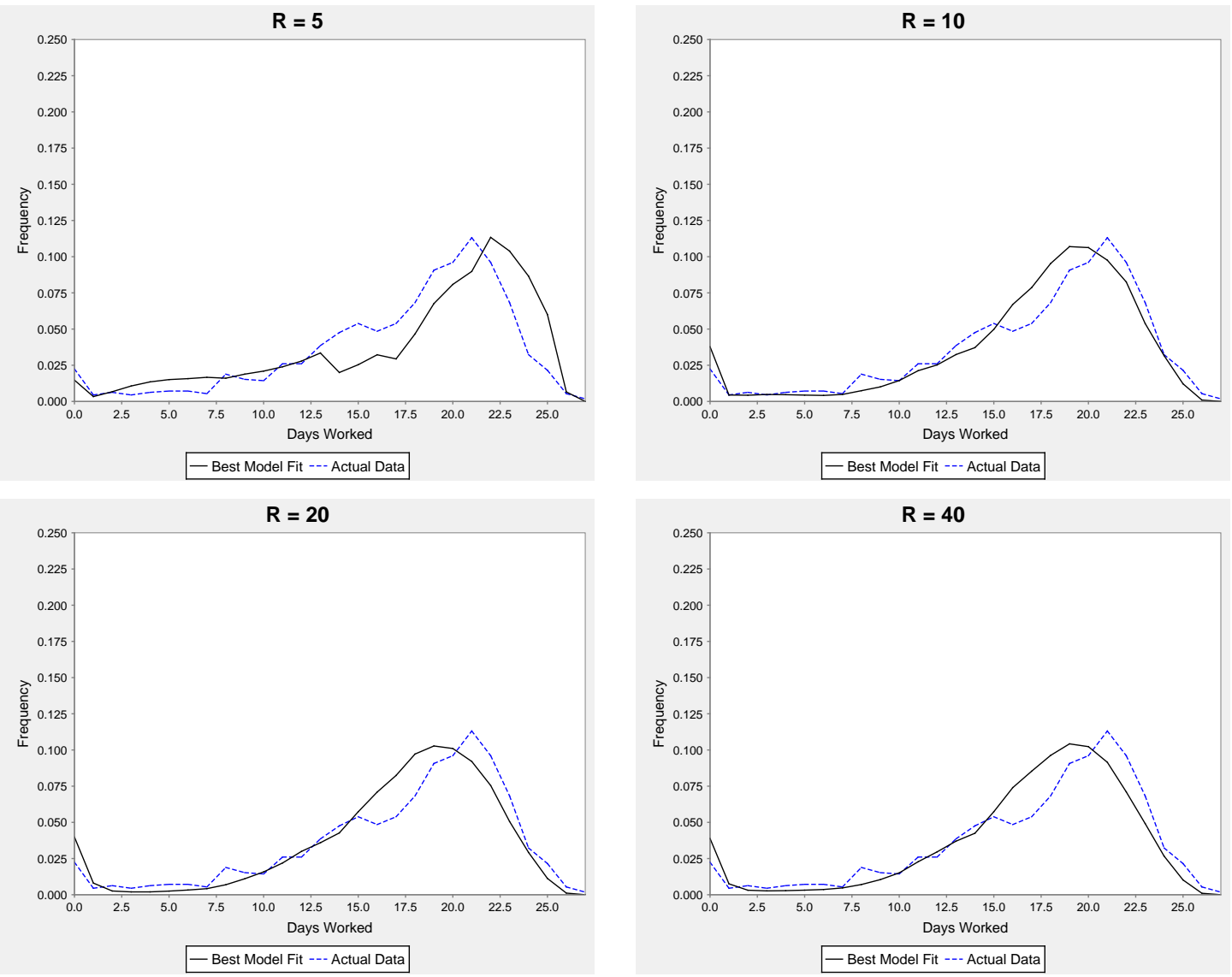
Figure 5: Predicted Distribution of Days Worked Under Out-of-Sample Compensation Scheme
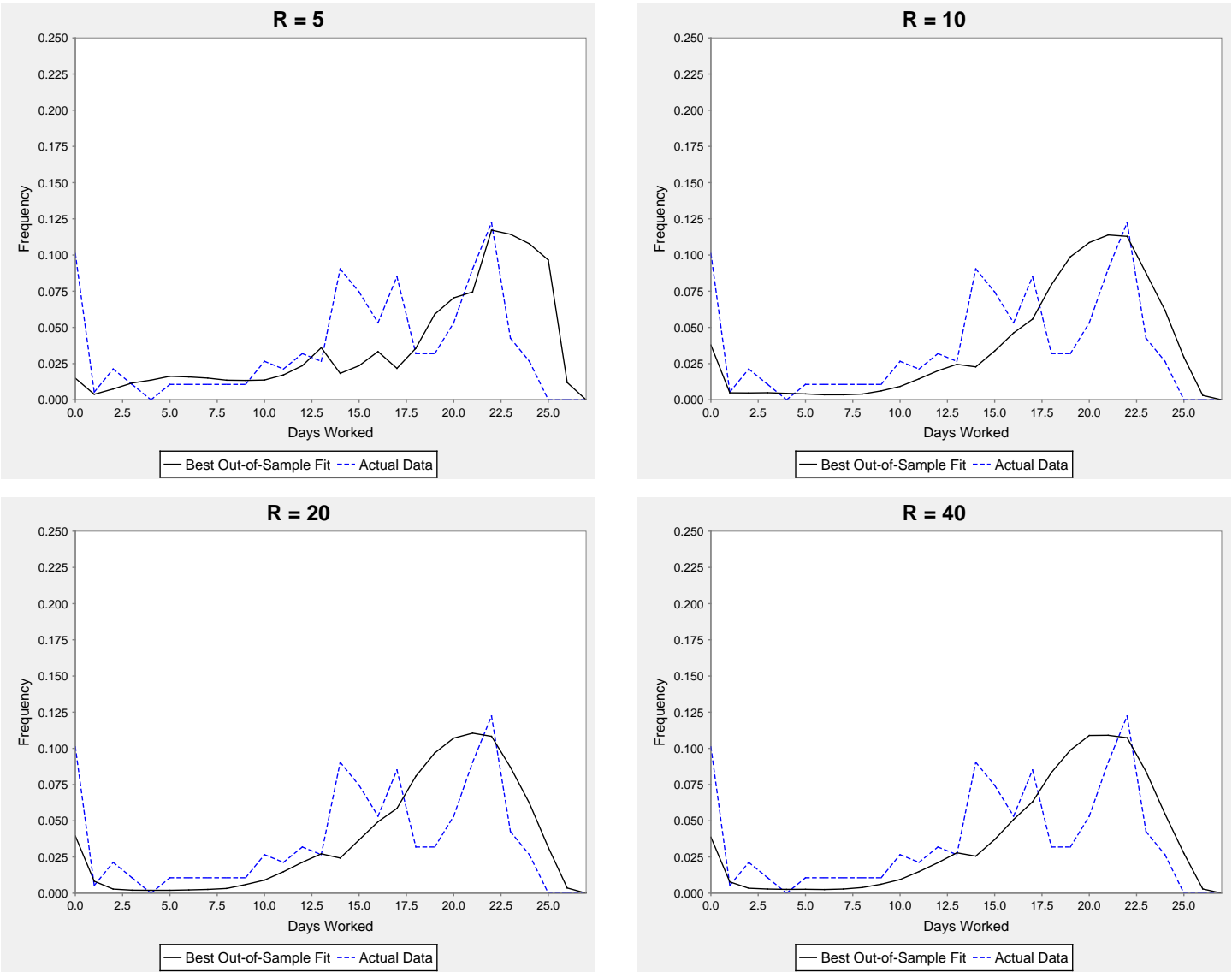
Table 1: Market-Share Approximations and the Number of Positive Weights, for Monte Carlo

\begin{tabular}{c|ccc|ccc}
\hline Design & \multicolumn{3}{|c|}{ RMSE } & \multicolumn{3}{c}{ \# of positive weights } \\
\hline Normal Mixture 2 & 0.000096 & 0.000042 & 0.000185 & 32 & 17 & 73 \\
& & & & & & \\
\hline Normal Mixture 4 & 0.000123 & 0.000045 & 0.000227 & 70 & 36 & 125 \\
& & & & & & \\
\hline Normal Mixture 6 & 0.000080 & 0.000043 & 0.000130 & 99 & 62 & 154 \\
& & & & & & \\
\hline
\end{tabular}

